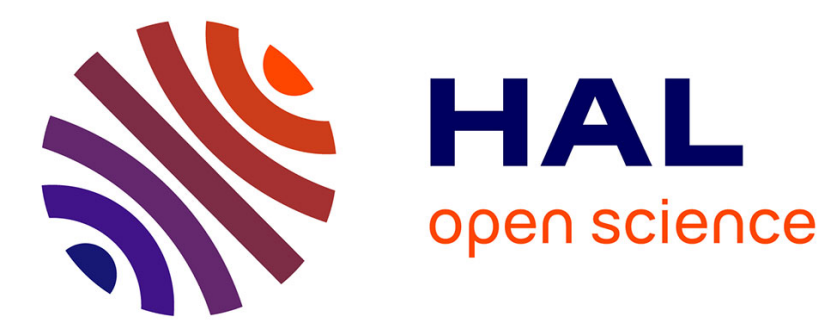

\title{
The integration of Gaussian noise by long-range amygdala inputs in frontal circuit promotes fear learning
}

Mattia Aime, Elisabete Augusto, Vladimir Kouskoff, Christelle Martin, Yann

Humeau, Nicolas Chenouard, Frédéric Gambino

\section{- To cite this version:}

Mattia Aime, Elisabete Augusto, Vladimir Kouskoff, Christelle Martin, Yann Humeau, et al.. The integration of Gaussian noise by long-range amygdala inputs in frontal circuit promotes fear learning. 2020. hal-03006642

\section{HAL Id: hal-03006642 \\ https://hal.science/hal-03006642}

Preprint submitted on 25 Nov 2020

HAL is a multi-disciplinary open access archive for the deposit and dissemination of scientific research documents, whether they are published or not. The documents may come from teaching and research institutions in France or abroad, or from public or private research centers.
L'archive ouverte pluridisciplinaire HAL, est destinée au dépôt et à la diffusion de documents scientifiques de niveau recherche, publiés ou non, émanant des établissements d'enseignement et de recherche français ou étrangers, des laboratoires publics ou privés. 


\section{The integration of Gaussian noise by long-range amygdala inputs in frontal circuit promotes fear learning}

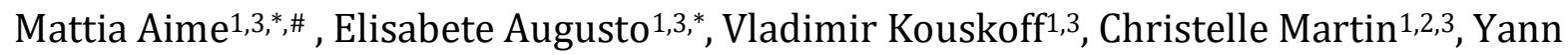

${ }^{1}$ Interdiscliplinary Institute for NeuroScience (IINS), CNRS, Centre Broca NouvelleAquitaine, 146, rue Léo-Saignat, 33076 Bordeaux, France.

2 Pole In Vivo.

3 UMR5297 and University of Bordeaux.

*, MA and EA contributed equally to this work

**, Corresponding Author: Frédéric Gambino (frederic.gambino@u-bordeaux.fr)

\#, Present address: University of Bern, Department of Neurology, Inselspital University Hospital Bern, Freiburgstrasse 18, CH-3010 Bern, Switzerland

Number of figures: 6

Number of Supplementary figures: 10

Number of Supplementary tables: 1

\section{ABSTRACT}

Survival depends on the ability of animals to select the appropriate behavior in response to threat and safety sensory cues. However, the synaptic and circuit mechanisms by which the brain learns to encode accurate predictors of threat and safety remain largely unexplored. Here, we show that frontal association cortex (FrA) pyramidal neurons integrate auditory cues and basolateral amygdala (BLA) inputs non-linearly in a NMDAR-dependent manner. We found that the response of FrA pyramidal neurons was more pronounced to Gaussian noise than to pure $8 \mathrm{kHz}$ tone, and that the activation of BLA-to-FrA axons was the strongest during safe periods in between conditioning pairings. Blocking BLA-to-FrA signaling specifically at the time of presentation of Gaussian noise (but not $8 \mathrm{kHz}$ tone) as a safety signal impaired the formation of auditory fear memories. Taken together, our data reveal a circuit mechanism that facilitates the formation of fear traces in the FrA, thus providing a new framework for probing discriminative learning and related disorders. 


\section{INTRODUCTION}

Discriminative learning is an important survival strategy that depends on the repeated contingency and contiguity between sensory cues (conditioned stimuli, CS) and the events (e.g., danger, safety) that they must predict (unconditioned stimuli, US) (Hall, 2002). It has been classically studied by using differential fear conditioning paradigms where two different auditory CS are positively (CS+) and negatively (CS-) paired in time with an aversive US (e.g., foot shock). This learning protocol is taken to assign appropriate emotional valence to the two incoming CSs (Hall, 2002; LeDoux, 2000; Likhtik and Paz, 2015), thereby providing an accurate representation of the environment by increasing discriminative skills between threat and safety signals. Whereas CS+ promotes conditioned fear responses (e.g., freezing behavior) when presented alone, CS- has been shown to serve as a learned safety predictor by reducing fear behavior and increasing positive affective responses (Rogan et al., 2005). While previous work has thoroughly investigated how CS+ generate fear responses (Dejean et al., 2016; Karalis et al., 2016)., it remains unclear whether and how the brain learns to encode CS- and thus discriminates between threat and safety.

The medial prefrontal cortex (mPFC) has appeared over the past decade as a critical region that shapes behaviors in response to both aversive and non-aversive environmental cues (Likhtik and Paz, 2015; Likhtik et al., 2014; Stujenske et al., 2014). These antagonistic effects of the mPFC possibly develop through specific interaction between its different subdivisions (i.e., prelimbic (PL) and infralimbic (IL) cortices) and the basolateral complex of the amygdala (BLA) (Senn et al., 2014; Sierra-Mercado et al., 2011; Vidal-Gonzalez et al., 2006). However, the mPFC does not receive direct sensory information neither from sensory cortical areas or from the thalamus (Hoover and Vertes, 2007), thereby supporting the idea that a higher-order neuronal network above the mPFC might encode specific memories that are later selected preferentially during recall together with its downstream cortical (e.g., PL or IL mPFC) or subcortical structures (e.g., BLA). Specifically, the superficial frontal association cortex (FrA) has been shown to contribute to memory formation during associative learning (Lai et al., 2012; Nakayama et al., 2015; Sacchetti et al., 2002). This region of the lateral part of the agranular cortex (AGl) (Paxinos and Watson, 2007; Uylings et al., 2003) receives inputs from the BLA (Lai et al., 2012; Mátyás et al., 2014; Nakayama et al., 2015) and sensory cortices (Hoover and Vertes, 2007; Zhang et al., 2016), and is non-reciprocally connected to the PL/IL 
subdivisions of the mPFC (Zhang et al., 2016), raising the possibility that it may function as a relay station during learning from sensory cortical areas and the BLA to the mPFC. However, whether and how the FrA integrates the variety of sensory information required for discriminative learning is not understood.

The involvement of the FrA in auditory fear conditioning has constantly been reported. For example, the pharmacological inactivation of FrA neurons alters both the expression and extinction of learned fear (Lai et al., 2012; Nakayama et al., 2015; Sacchetti et al., 2002). Recently, fear conditioning and extinction have been shown to induce dendritic spine elimination and formation in the FrA, respectively (Lai et al., 2012). Importantly, this phenomenon occurs within the same dendritic branch, supporting the idea that a unique FrA circuit could form memory traces with distinct emotional values. Nonetheless, no previous evidence has demonstrated the contribution of the FrA in the encoding of incoming sensory cues as threat or safety predictors and, if so, how such a process may be controlled by inputs from the BLA.

To address the possible role of the FrA in discriminative learning and the mechanisms behind it, we investigated auditory-evoked computations of layers II/III FrA pyramidal neurons, as well as the dynamics of long-range projections from the BLA during the acquisition and recall of fear memory traces. By using two-photon (2P) calcium imaging in head-restrained mice, in vivo whole cell recordings and optogenetics, we found that FrA pyramidal neurons process auditory tones based on their spectral properties. Unlike pure frequency tones, Gaussian noise produced somatic and dendritic depolarizations in FrA pyramidal neurons. The photo-stimulation of BLA-to-FrA neurons resulted in the supra-linear integration of auditory tones. During conditioning, the activity of BLA-to-FrA axons was stronger between CS+/US pairings that is, when CS- is presented) than during pairings. Inhibiting these axons during CS- impaired auditory fear learning but only when Gaussian noise is used as CS-. Taken together, our data support the idea that FrA and BLA-to-FrA neurons gate learning by promoting the integration of non-conditioned Gaussian noise (i.e., not paired to the footshock). In conclusion, the study reveals a potent dendritic mechanism for encoding predictors in the FrA, and thus extends the cortical framework for probing discriminative learning and related disorders. 


\section{RESULTS}

\section{Auditory tones recruit NMDARs conductances in FrA L2/3 pyramidal neurons}

We performed somatic whole-cell recordings in anesthetized naive animals and characterized the activation of FrA L2 pyramidal cells to different sounds by presenting a pure tone $(8 \mathrm{kHz})$ and a broad-band mixture of pure tones (white Gaussian noise, $W G N$ ) (Fig. 1), which have been widely used in previous fear conditioning studies (Dejean et al., 2016; Grosso et al., 2015; Karalis et al., 2016; LeDoux, 2000; Likhtik et al., 2014; Park et al., 2016; Senn et al., 2014; Stujenske et al., 2014). For each recorded cell, membrane potential $(\mathrm{Vm})$ was monitored prior to, during and after the random presentation of both tones, each consisting of 27 pips ( $50 \mathrm{~ms}, 0.9 \mathrm{~Hz}$ for $30 \mathrm{~s}$ ) (Fig. 1B-F). Membrane potential spontaneously fluctuated between up and down states (Fig. 1B). Therefore, to detect changes in Vm specifically induced by the auditory stimulation, we computed the cumulative depolarization over time $(\mathrm{cVm})$, from which was then subtracted the linear regression calculated during the baseline period prior to auditory stimulation $(\mathrm{cVm}$ change) (Fig. 1C, D; Fig. S1A, B). This allowed us to minimize the variability related to spontaneous activity and thus compare evoked depolarizations under different conditions.

WGN evoked a subthreshold depolarization in naive animals $(27.6 \pm 4 \mathrm{mV}, \mathrm{n}=22)$ that lasted for at least $30 \mathrm{sec}$ after the end of the stimulation (32.5 $5 \mathrm{mV}, \mathrm{n}=22$ ) (Fig. 1C, D). This change in Vm was abolished by the application of the NMDAR antagonist D(-)-2amino-5-phosphonovaleric acid (dAP5) or the presence of the NMDAR open-channel blocker MK-801 in the intracellular solution (end of the stimulation; control: $27.6 \pm 4 \mathrm{mV}$, $\mathrm{n}=22$; +dAP5: $-13.4 \pm 5 \mathrm{mV}, \mathrm{n}=14$; +iMK801: $-2.2 \pm 2 \mathrm{mV}, \mathrm{n}=5 ; \mathrm{p}<0.001$, anova) (Fig. 1D; Fig. S1C-F). This suggests that $W G N$ generates NMDAR-mediated depolarization that appears similar to somatic plateau potentials (Gambino et al., 2014).

In contrast, pure auditory tone $(8 \mathrm{kHz})$ did not seem to affect membrane potential either during ( $8 \mathrm{kHz}: 1.2 \pm 4 \mathrm{mV}$; $W G N$ : $27.6 \pm 4 \mathrm{mV}$; $\mathrm{n}=22$, $\mathrm{p}<0.001$, paired t-test) or after auditory stimulation ( $8 \mathrm{kHz}:-3.8 \pm 3 \mathrm{mV}$; WGN: $32.5 \pm 5 \mathrm{mV} ; \mathrm{n}=22, \mathrm{p}<0.001$, paired $t$-test). Nonetheless, we found that $8 \mathrm{kHz}$ tones hyperpolarized pyramidal neurons in the presence of dAP5 or iMK801 (end of stimulation; control: $1.16 \pm 3.9 \mathrm{mV}, \mathrm{n}=22$; +dAP5: $16.7 \pm 4 \mathrm{mV}, \mathrm{n}=10$; +iMK801: $-21 \pm 3.5 \mathrm{mV}, \mathrm{n}=5 ; \mathrm{p}=0.004$, anova), thus revealing an 
and $8 \mathrm{kHz}$ tones hyperpolarized FrA neurons to a similar level under NMDAR blockade $(W G N:-10.5 \pm 3.6 \mathrm{mV}, \mathrm{n}=19 ; 8 \mathrm{kHz}:-18.2 \pm 2.9 \mathrm{mV}, \mathrm{n}=15 ; \mathrm{p}=0.125, t$-test; dAP5 and iMK801 conditions pooled together), suggesting that a fraction of the NMDAR conductances recruited by auditory stimulations is masked by non-specific inhibition under control conditions (Doron et al., 2017). However, on average, the NMDAR-mediated component of the evoked $\mathrm{cVm}$ change was much larger in response to WGN, indicating that WGN recruited more NMDAR conductances than pure auditory tones (WGN: $33.1 \pm$ $4.2 \mathrm{mV}$; $8 \mathrm{kHz}$ : $9.7 \pm 3.4 \mathrm{mV}$; n=5; p=0.017, paired $t$-test) (Fig. S1G-J). Altogether, our data indicate that, during anesthesia, Gaussian noise activates enough NMDAR-mediated synaptic inputs to produce sustained depolarization of the cell body.

\section{Auditory tones generate local calcium events in distal FrA dendrites}

NMDARs conductances confer unique computational capabilities to pyramidal neurons by operating supra-linear signaling in dendrites (Antic et al., 2010a; Major et al., 2013). These NMDAR-mediated events are highly localized to a small dendritic segment, but can spread towards the soma to produce plateau potentials (Gambino et al., 2014; Palmer et al., 2014). Thus, we investigated whether auditory tones generate local dendritic events. We infected mice with an AAV9-Syn-flex-GCaMP6s together with a 1:10000 dilution of AAV1-hSyn-cre (Fig. 2A) in order to obtain a sparse labeling with few neurons expressing GCaMP6s. The activity of non-overlapping distal dendritic branches was imaged in superficial layer 1 through an implanted cranial window in anesthetized (139 dendrites from 5 mice; Fig. 2B-D) and awake mice (104 dendrites from 8 mice; Fig. 2B, E, F). We isolated calcium transients and segregated them based on their spatial spread along individual dendrites (Fig. 2C; Fig. S2A-C).

We detected calcium transients in apical dendritic tufts of mice that were anesthetized with isoflurane (1.5\%). These calcium events occurred both spontaneously (i.e. during baseline prior to stimulation) and upon auditory stimulation. The presentation of WGN evoked more events (WGN: 409; 8 kHz: 139; 2 kHz: 102; $\mathrm{p}<0.001$, McNemar's $\chi^{2}$ test), with a significantly higher number of local dendritic events per dendrite as compared to pure frequency auditory tones $(W G N$ : $3.28 \pm 0.4 ; 8 \mathrm{kHz}: 1.19 \pm 0.3 ; 2 \mathrm{kHz}$ : $1.26 \pm 0.25 ; \mathrm{n}=8 ; \mathrm{p}<0.001$, anova) (Fig. 2C, D). $8 \mathrm{kHz}$ and $2 \mathrm{kHz}$ tones did not evoke calcium transients more frequently than baseline (Fig. 2D). Although it remains possible that pure 
tones activate basal dendrites, our data demonstrate that, during anesthesia, apical dendrites located in L1 are specifically activated by WGN.

During anesthesia, auditory-evoked calcium events were mostly local, with a full width at half maximum (fwhm: $13.5 \pm$ (s.d.) $13 \mu \mathrm{m}, \mathrm{n}=650$ events) that fell into the spatial range of NMDAR-mediated spikes(Gambino et al., 2014; Palmer et al., 2014) (Fig. 2G). Global calcium transients with a longer spatial extent (fwhm $\geq 50 \mu \mathrm{m} ; 81 \pm$ (s.d.) $35 \mu \mathrm{m}$, $\mathrm{n}=313$ events) were additionally observed in awake mice (Fig. 2E, H). These events, which could reflect backpropagating somatic action potentials, occurred independently of the nature of the auditory tone (Fig. S2). In contrast, most of the local calcium transients were concurrent with WGN (WGN: 339; 8 kHz: 218; 2 kHz: 253; p<0.001, McNemar's $\chi^{2}$ test). Again, and as expected, $W G N$ evoked significantly more local events per dendrite than pure tones (WGN: $3.37 \pm 0.3 ; 8 \mathrm{kHz}: 2.49 \pm 0.2 ; 2 \mathrm{kHz}: 2.01 \pm 0.2 ; \mathrm{n}=5 ; \mathrm{p}<0.001$, anova) (Fig. 2F), thus mirroring what we observed during anesthesia (Fig. 2I). Nevertheless, 8 $\mathrm{kHz}$ and $2 \mathrm{kHz}$ tones generated more local events as compared to baseline (Fig. 2F), suggesting that anesthesia might reduce dendritic signaling of pure frequency auditory inputs (1.22 \pm 0.3 vs. $2.24 \pm 0.2 ; \mathrm{p}=0.037)$ (Fig. 2I). However, the specific effect of $W G N$ in dendrites was not attributable to noise-induced pain, since exploratory motor behaviors were not affected by WGN or pure tones (Fig. S3).

Taken together, our results show that FrA pyramidal neurons process auditory tones differently according to their spectral properties in both anesthetized and awake mice. This occurs at the subthreshold level with Gaussian auditory tones being more efficient in producing somatic, plateau-like depolarizations (Fig. 1) and local dendritic events within the same tuft dendritic branch (Fig. 2) than pure tones.

\section{Non-conditioned Gaussian noise promotes fear memories in FrA circuit}

WGN and pure frequency tones produce distinct forms of synaptic plasticity during auditory fear conditioning (Park et al., 2016). This raises the possibility they might play a specific role during learning (Grosso et al., 2015; Park et al., 2016). To test this hypothesis, we injected mice bilaterally with an AAV expressing the light-activated proton pump archaerhodopsin (AAV9.CaMKII.ArchT.GFP, $\mathrm{n}=40$; or AAV9.CamKII.eGFP for controls, $\mathrm{n}=35$ ) into the FrA. We transiently inhibited the activity of the FrA during learning with light through implanted optical fibers (Fig. 3A, B). Auditory fear conditioning (FC) was induced by using a classical discriminative protocol, during which five auditory stimuli 
(each consisting of $27 W G N$ or $8 \mathrm{kHz}$ pips, $50 \mathrm{~ms}, 0.9 \mathrm{~Hz}$ for $30 \mathrm{~s}$ ) were positively (CS+) or negatively (CS-) paired with the delivery of a mild electrical shock $(0.6 \mathrm{~mA})$ to the paws in a pseudorandom order (Fig. S4). The auditory tones ( $8 \mathrm{kHz}$ and $W G N$ ) used for CS+ and CS- during conditioning were counterbalanced across mice (protocol 1, CS+/CS-: 8 $\mathrm{kHz} / W G N$ respectively; protocol 2, $\mathrm{CS}+/ \mathrm{CS}-: W_{G N} / 8 \mathrm{kHz}$ respectively) (Fig. S4A, B), and learning was tested $24 \mathrm{~h}$ later during recall by measuring cue-induced freezing in a novel context (Fig. 3B). Mice were classified as learners (learning+) when the learning index was higher than $20 \%$ during recall (Fig. S4E, F).

First, we suppressed the activity of FrA L2/3 neurons by delivering light each time a CS+ was presented during conditioning (Fig. 3C, D). Surprisingly, neither the fraction of mice that learned the cue-shock association (protocol 1, GFP: 75\%, n=8, ArchT: 87\%, n=8, p=0.522; protocol 2, GFP: 87\%, n=8, ArchT: 65\%, n=8, p=0.248, Pearson's $\chi^{2}$ test), nor the percentage of freezing induced by CS+ during recall (GFP: $48.5 \pm 5 \%, \mathrm{n}=16$; ArchT: $38.5 \pm$ $5 \%, n=16 ; \mathrm{p}=0.2$ ) were affected by the inhibition of FrA neurons (Fig 3D-F). In contrast, ArchT stimulation during CS- presentation (Fig. 3G, H) significantly decreased the fraction of mice that were conditioned. However, this occurred only when $W G N$ was used as CS- (protocol 1, GFP: 75\%, n=12, ArchT: 30\%, n=13, p=0.02; protocol 2, GFP: 85\%, n=7, ArchT: 91\%, $\mathrm{n}=11, \mathrm{p}=0.7$, Pearson's $\chi^{2}$ test) (Fig. 3I). In those mice, the magnitude of CS+ evoked freezing responses was significantly lower during recall than in control mice (protocol 1, GFP: $51 \pm 7 \%, \mathrm{n}=12$; ArchT: $24.5 \pm 5 \%, \mathrm{n}=13$; p=0.002, post-hoc Holm-Sidak test) (Fig. 3J).

These data demonstrate that when $W G N$ is paired negatively to footshock during conditioning, it promotes fear memory traces in the FrA circuit, thus confirming the specific nature of complex auditory cues during learning (Grosso et al., 2015; Park et al., 2016). To further clarify the synaptic mechanisms involved, we measured auditoryevoked dendritic and somatic responses following conditioning protocol 1. As compared to naïve mice (Fig. 2), WGN (CS-) generated less local dendritic activation during recall (protocol 1; naive: $3.37 \pm 0.3, \mathrm{n}=9 ; F C: 1.88 \pm 0.3, \mathrm{n}=5$ mice; $\mathrm{p}=0.003$ ) (Fig. S5). In contrast, local dendritic events evoked by a conditioned cue $(8 \mathrm{kHz})$ or cues that were not presented during conditioning $(2 \mathrm{kHz})$ were not altered, indicating that fear learning specifically affected $W G N$-mediated dendritic signaling. In addition, $W G N$-induced somatic plateau potentials were reduced in conditioned animals in a learning-dependent manner, suggesting that NMDAR-dependent plasticity mechanisms in FrA neurons were 
engaged during fear learning (Basu et al., 2016; Lai et al., 2012; Rioult-Pedotti et al., 2000) (Fig. S6).

\section{BLA-to-FrA axons are recruited during fear conditioning}

The above mentioned results indicate that the FrA is required for fear learning and guides behaviors by integrating non-conditioned complex auditory cues during conditioning. Given the well-established role of the BLA and its cortical projection during the acquisition and expression of auditory cue fear learning (LeDoux, 2000; Likhtik and Paz, 2015; Likhtik et al., 2014; Nakayama et al., 2015; Senn et al., 2014; Stujenske et al., 2014), we next investigated the information transmitted from the BLA to the FrA (Lai et al., 2012; Mátyás et al., 2014; Nakayama et al., 2015) during conditioning. We injected a virus expressing the genetically encoded calcium indicator GCaMP6f into the right BLA and imaged axonal $\mathrm{Ca}^{2+}$ responses in the superficial $\mathrm{L} 1$ of the right FrA of awake headrestrained mice during fear conditioning (Fig. 4A, B). Results confirmed that BLA neurons project to the superficial layer 1 of the ipsilateral FrA $(<150 \mu \mathrm{m})$ (Lai et al., 2012; Mátyás et al., 2014; Nakayama et al., 2015) (Fig. 4B; see also Fig. S8). We conditioned awake mice $(\mathrm{n}=10)$ under the 2-photon microscope by using the same counterbalanced protocols as described previously (protocol 1, n=7; protocol 2, n=3) (Fig. S4). GCaMP6f calcium transients $\left(\Delta \mathrm{F} / \mathrm{F}_{0}\right)$ provided a direct measure of the activation of BLA neurons projecting to the FrA (Fig. 4B). Again, learning was tested $24 \mathrm{~h}$ later and quantified by the percentage of freezing (Fig. 4D). We then compared the activity of individual boutons between mice that learned (learning,$+ \mathrm{n}=5$ ) and those that failed to learn (learning-, $\mathrm{n}=5$ ).

As a first metric to quantify the activity of BLA-to-FrA axons, we measured the number of calcium transients in individual boutons observed during baseline and in conditioning pairings. While the activity of individual BLA boutons in FrA was relatively low at rest, it increased significantly upon successive pairings (Fig. 4D). This occurred independently of the conditioning protocol and only in mice that learned (learning + , all CS+: $1.73 \pm 0.3$, all CS-: $2.07 \pm 0.45, n=5$; learning-, all CS+: $0.62 \pm 0.08$, all CS-: $0.77 \pm 0.13$, $\mathrm{n}=5$; $\mathrm{p}=0.015$ ) (Fig. 4D-F; Fig. S7). Interestingly, it also never occurred before the end of the first US presentation. Indeed, the number of transients observed during the first CS+ (i.e. before the delivery of the first US) was significantly lower than the other CS that were paired with the US (learning + , CS/No US: $1.03 \pm 0.15$, CS/US: $1.97 \pm 0.4, n=5, p=0.011$; learning-: CS/No US: $0.74 \pm 0.05$, CS/US: $0.68 \pm 0.08, n=5, p=0.863$ ) (Fig. 4F). As a 
consequence, the activity of boutons measured during the first CS- was always higher than during the first $\mathrm{CS}+($ learning $+, \mathrm{CS}+1: 1.03 \pm 0.15, \mathrm{CS}-1: 2.03 \pm 0.4, \mathrm{n}=5, \mathrm{p}=0.003$; learning$, C S+1: 0.74 \pm 0.05, C S-1: 0.88 \pm 0.09, n=5, p=0.425$ ) (arrow in Fig. 4F). These data suggest that neither the tone alone nor the foot shock influence the activity of BLA-to-FrA axons. This was corroborated by the observation that BLA-to-FrA axons were never activated by auditory stimulations in naive mice (i.e. before fear conditioning) (Fig. S7). Instead, our data support the idea that BLA axons projecting to the FrA convey information about learning, i.e. the CS+/US association itself rather than about the nature of the auditory tones (Nakayama et al., 2015).

Then, we summed the amplitude of all calcium transients detected during each CS presentation (cumulative $\Delta \mathrm{F} / \mathrm{F}_{0}$ averaged across all CS+ or CS-). The averaged cumulative $\Delta \mathrm{F} / \mathrm{F}_{0}$ measured during CS- was always higher than during CS+ (CS-: $0.27 \pm 0.07 \Delta \mathrm{F} / \mathrm{F}_{0}$, $\mathrm{CS}+\mathrm{0} 0.21 \pm 0.06 \Delta \mathrm{F} / \mathrm{F}_{0}, \mathrm{n}=10 ; \mathrm{p}=0.012$ ), revealing that the overall activity of BLA-to-FrA axons was stronger between conditioning trials, notably at the time when CS- occurred (Fig. 4G). Importantly, the difference between CS+ and CS- related axonal activity ( $\Delta$ cumulative: $\Delta \mathrm{F} / \mathrm{F}_{0} \mathrm{Cs}_{-}-\Delta \mathrm{F} / \mathrm{F}_{0 \mathrm{cs}+}$ ) was significantly higher in mice that learned the association than in non-learners (learningt: $0.1 \pm 0.02 \Delta$ cumulative, $\mathrm{n}=5$; learning-: 0.01 $\pm 0.008 \Delta$ cumulative, $n=5 ; p=0.013$, $t$-test) (Fig. 4G). In fact, when plotted as a function of freezing percentage observed during recall, $\Delta$ cumulative correlated positively with learning performance $\left(r^{2}=0.89, p<0.001\right)$ (Fig. 4H), suggesting that the level of activity of BLA-to-FrA axons during CS- is critical for the acquisition of fear memories.

Non-linear interaction in FrA L2 /3 pyramidal neurons between segregated BLA and auditory inputs

The activation of BLA neurons instructs prefrontal circuits during learning and memory recall (Klavir et al., 2017; Nakayama et al., 2015; Stujenske et al., 2014). However, the optical activation of the BLA alone is not sufficient to produce learned associations (Johansen et al., 2010). Therefore, we hypothesized that the activation of BLA axons during CS- (Fig. 4), along with the synaptic non-linearities evoked by WGN (Fig. 1 and 2), could gate fear learning (Fig. 3) by controlling L2/3 FrA pyramidal neurons through their projections into L1.

To test this hypothesis, we first addressed the properties of BLA-to-FrA synapses in naive mice. We expressed the recombinant light-gated ion channel channelrhodopsin- 
2-YFP (ChR2; AAV9-CamKIIa-hChR2-eYFP) in the BLA and performed intracellular recordings in L2/3 FrA neurons from naive mice (Fig. 5). BLA neurons expressing ChR2 projected to the superficial layer 1 of the ipsilateral FrA $(<150 \mu \mathrm{m})$ (Fig. 5A; Fig. S8A-C), thereby most likely contacting dendritic tufts of L2/3 pyramidal neurons. Local photostimulation of ChR2-BLA axons in acute slices produced excitatory postsynaptic current (EPSC) in FrA pyramidal neurons with short latencies $(3.5 \pm 0.36 \mathrm{~ms}, \mathrm{n}=9)$ and low jitter $(0.289 \pm 0.04 \mathrm{~ms}, \mathrm{n}=9)$, suggesting that a fraction of BLA neurons are connected monosynaptically to L2/3 FrA pyramidal neurons (Fig. S8D, E) (Klavir et al., 2017). In vivo, the photostimulation of BLA neurons with an implanted optical fiber produced plateau-like depolarizations in all FrA neurons (averaged peak amplitude: $6.2 \pm 1.2$ $\mathrm{mV}^{*} \mathrm{sec}$, full-width at half-max (fwhm): $551 \pm 80 \mathrm{~ms}$; $\mathrm{n}=13$ ). However, BLA-to-FrA inputs were mostly undetectable and became visible only when the stimulation was delivered during down states (Fig. S8F, G). When detected, the distribution of amplitudes across cells suggested that, on average, they were highly variable (range of amplitude: $0.4 \mathrm{mV}$ $14.5 \mathrm{mV}$ ) (Fig. S8H). The rhythmic stimulation of ChR2-expressing BLA neurons at $0.9 \mathrm{~Hz}$ for $30 \mathrm{sec}$ (27 square pulses, 50ms) (Fig. 5B, C), a protocol that mimicked the pattern of auditory stimuli, generated modest cumulative depolarization $(8 \pm 4 \mathrm{mV} ; \mathrm{n}=21)$. Taken together, our data indicate that BLA-to-FrA synapses are likely to be weak and unreliable.

We next investigated the effect of BLA activation during auditory stimulation on L2/3 FrA pyramidal neurons (Fig. 5D-I). We first verified that $W G N$ alone was able to activate FrA pyramidal neurons in mice chronically implanted with optical fibers. Similar to the effect of auditory stimulation in non-implanted mice (Fig. S9A), $W G N$ but not $8 \mathrm{kHz}$ evoked a long-lasting subthreshold depolarization ( $W G N$ : $18.9 \pm 7 \mathrm{mV}, \mathrm{n}=11 ; 8 \mathrm{kHz}$ : $-9.7 \pm$ $3 \mathrm{mV}, \mathrm{n}=8 ; \mathrm{p}=0.005$; $t$-test). The coincident photo-activation of BLA neurons during the presentation of $W G N$ resulted in somatic responses (observed $\mathrm{cVm}$ ) that were significantly higher than the arithmetic sum (expected cVm) of individual depolarizations evoked by the stimulation of BLA or auditory tones alone (observed cVm: $44 \pm 6.4 \mathrm{mV}$, expected cVm: $18 \pm 8.8 \mathrm{mV}, \mathrm{n}=11$; p=0.002, paired t-test) (Fig. 5D, E, H; Fig. S9B, C). We then plotted the observed vs. expected cVm change and found that the observed $c V m$ exceeded the expected $\mathrm{cVm}$, indicating that the interaction in FrA neurons between BLA and WGN-related inputs was clearly supralinear (Spruston and Kath, 2004; Tran-VanMinh et al., 2015) (Fig. 5H). Supra-linear operations have been shown to depend on active dendritic conductances (Spruston and Kath, 2004; Tran-Van-Minh et al., 2015). 
Accordingly, we found that the application of dAP5 $(1 \mathrm{mM})$ to the cortical surface blocked the effect of BLA activation during WGN (BLA+WGN: $39 \pm 6.2 \mathrm{mV}, \mathrm{n}=13$; $\mathrm{BLA}+W G N /+\mathrm{dAP} 5:-26.6 \pm 6.7 \mathrm{mV}, \mathrm{n}=7 ; \mathrm{p}<0.001 ; t$-test), indicating that NMDARs are involved in the supra-linear integration in FrA neurons (Fig. S9F). The photo-activation of BLA also significantly affected the cumulative potential evoked by $8 \mathrm{kHz}$ (8 $\mathrm{kHz}: 9.7 \pm$ $3.3 \mathrm{mV}$ vs. $8 \mathrm{kHz}+\mathrm{BLA}: 16.6 \pm 3.1 \mathrm{mV} ; \mathrm{n}=8$; p<0.001; paired t-test) (Fig. 5F, G; Fig. S9D, E). Although supra-linear (observed cVm: $16.6 \pm 3.1 \mathrm{mV}$, expected cVm: $-2.4 \pm 3.3 \mathrm{mV}, \mathrm{n}=8$; $\mathrm{p}<0.001$, paired t-test), the BLA+8 $\mathrm{kHz}$ integration remained significantly lower than the supra-linearity generated by BLA+WGN (observed cVm; WGN: $44 \pm 6.4 \mathrm{mV}, \mathrm{n}=11 ; 8 \mathrm{kHz}$ : $16.6 \pm 3.1 \mathrm{mV}, \mathrm{n}=8 ; \mathrm{p}=0.003$, t-test). This indicates that BLA axons are necessary to produce non-linear integration of auditory inputs in FrA neurons, which is stronger for Gaussian noise than for pure tones.

\section{BLA-to-FrA non-linear integration of Gaussian noise gates fear learning}

We next examined whether this BLA-mediated, non-linear integration of auditory inputs in FrA pyramidal neurons could play a role in the acquisition of fear memories. This question was addressed by silencing specifically the BLA-to-FrA axons during conditioning with optogenetics (Fig. 6 and Fig. S10). Mice were injected bilaterally with a retrograde Cav-2-CMV-Cre (Hnasko et al., 2006) into the FrA together with either AAV9. CBA.Flex.ArchT.GFP (ArchT-expressing mice, $n=24$ ) or AAV9.CAG.Flex.eGFP (control GFPexpressing mice, $n=25$ ) into BLA bilaterally (Fig. 6A). This resulted in the restricted expression of the light-driven inhibitory proton pump ArchT (or GFP for controls) in a target-specific fraction of BLA neurons that project to the FrA (Fig. 6B-D). Mice were then submitted to auditory fear conditioning, and we analyzed the impact of opto-stimulation on freezing behaviors for each counter-balanced protocol (Fig. S10).

We found that the time-locked suppression of BLA-to-FrA communication during negatively-paired WGN (CS-, protocol 1b) significantly decreased the fraction of ArchTmice that learned the association (GFP: 87.5\%; ArchT: 22\%, p=0.005, Pearson's $\chi^{2}$ test) and freezing behaviors upon subsequent CS+ $(8 \mathrm{kHz})$ presentation (GFP: $55.9 \pm 8 \%, \mathrm{n}=8$; ArchT: $34.9 \pm 3 \%, n=7 ;$ p=0.037, t-test) (Fig. 6E, F; Fig. S10). These results were similar to those obtained when FrA neurons were inhibited during conditioning (Fig. 3; Fig. S10), thus confirming that negatively paired $W G N$ participates in the formation of fear traces. Surprisingly, however, ArchT-expressing mice showed increased fear responses in 
response to CS- during recall (GFP: $5.3 \pm 1 \%, \mathrm{n}=8$; ArchT: $13.7 \pm 4.5 \%, \mathrm{n}=7 ; \mathrm{p}=0.04, t$ test) (Fig. 6F). In agreement with previous studies(Likhtik and Paz, 2015; Likhtik et al., 2014; Sangha et al., 2013; Senn et al., 2014; Stujenske et al., 2014), this suggests that CSalso acquires safety properties through the activation of a specific population of BLA neurons.

In contrast, neither the fraction of mice that learned (GFP: 78\%; ArchT: 44\%, $\mathrm{p}=0.145$, Pearson's $\chi^{2}$ test) nor freezing responses during recall (CS+; GFP: $34.8 \pm 3.9, \mathrm{n}=9$; ArchT: $29.3 \pm 8, n=9 ; p=0.530$, $t$-test) were affected by blocking the activity of BLA-to-FrA axons during negatively-paired $8 \mathrm{kHz}$ (CS-, protocol 2b) (Fig. 6E, G). Similar results were obtained when photo-stimulation was administered during CS+ (WGN, protocol 2a) (Fig. S10). Altogether, our data support the idea that if Gaussian noise is presented when the activity of BLA neurons is the strongest (i.e. between conditioning trials, Fig. 4), it facilitates discriminative learning. 


\section{DISCUSSION}

The present study investigates the role of the BLA-to-FrA circuit in the integration of auditory cues, and how this process participates in the acquisition of fear traces during conditioning. Taken together, our data demonstrate that rather than being an obstacle, Gaussian noise facilitates auditory fear learning when not paired to the foot shock (i.e. delivered between conditioning trials), thereby confirming the sophisticated nature of differential conditioning protocols (Grosso et al., 2015; Hall, 2002; McDonnell and Abbott, 2009). This is likely due to: 1) the specific dendritic and somatic responses of FrA pyramidal neurons to $W G N$; 2) the activation of BLA-to-FrA axons between conditioning pairings, which might support 3) the non-linear integration of Gaussian noise in FrA neurons. Unlike $W G N$, none of the optogenetic manipulations aiming at altering pure tone processing in FrA and BLA during conditioning had an effect on fear learning. Although our results might also depend on the specific association between pure and Gaussian auditory tones during conditioning, they bring new conceptual perspectives to central questions regarding how frontal circuits contribute to learning, thus going beyond the BLA-mPFC interactions classically described in fear learning studies (Likhtik and Paz, 2015).

Accumulating evidence from anatomical and functional studies has demontrated that despite its pivotal role in the acquisition and expression of associations between sensory stimuli and the emotional valence of these stimuli (Laviolette et al., 2005; Roesch et al., 2010), the mPFC is not directly involved in sensory processing (Hoover and Vertes, 2007; Martin-Cortecero and Nuñez, 2016; Zhang et al., 2016). In contrast, due to its anatomical connections with distributed cortical and subcortical regions (Hoover and Vertes, 2007; Zhang et al., 2016), the FrA might serve as a hub that coordinates incoming sensory information before reaching the mPFC. Here, we show that it is indeed required for fear learning in a rather unconventional way, and further clarify the underlying synaptic mechanisms. To our knowledge, this is the first demonstration that auditory sensory stimulation produces NMDAR-dependent depolarization in FrA L2/3 pyramidal neurons and activates their dendrites during both anesthesia and wakefulness. These somatic and dendritic events were more pronounced with Gaussian noise than with pure frequency auditory stimulation. Although we cannot rule out that $W G N$ tones are structured and further abstracted throughout the entire auditory system (Deneux et al., 2016), the simplest explanation is that frequency-tuned spines are distributed widely and 
heterogeneously throughout the same FrA dendrite, as already reported in the auditory cortex of anesthetized mice (Chen et al., 2011). As a consequence, the multiple frequencies composing WGN might promote the activation of a dense pattern of neighboring spines that in turn facilitate the generation and propagation of local non-linear events towards the soma(Antic et al., 2010b). Multiple calcium transients occurring simultaneously in multiple dendritic branches are necessary to affect somatic voltage (Palmer et al., 2014). Both those findings and ours suggest that WGN-induced depolarization is the consequence of multiple calcium events that occur in different dendritic branches of the same neuron. In contrast, pure-frequency tone appeared unable to activate enough branches simultaneously, thereby making the alteration of somatic voltage less probable.

The FrA and the BLA are anatomically interconnected (Lai et al., 2012; Mátyás et al., 2014; Nakayama et al., 2015). However, the functional properties of these connections remain unknown, notably during learning. Our results confirm that BLA neurons project to the superficial layer of the FrA, thereby most likely contacting dendrites of L2/3 pyramidal neurons. The photo-stimulation of ChR2-expressing BLA neurons produced plateau-like depolarizations that were rather weak and unreliable. It thus seems unlikely that they can summate during rhythmic activation to create favorable conditions for the integration of coincident sensory-driven inputs (Antic et al., 2010b; Brandalise et al., 2016; Gambino et al., 2014; Xu et al., 2012). Alternatively, it is possible that the modest activation of BLA synapses in FrA apical dendrites facilitates or gates the propagation towards the soma of tone-evoked dendritic events (Jarsky et al., 2005). In agreement, we observed that the coincident activation of BLA-to-FrA inputs increased both WGN and 8 $k H z$-evoked depolarization non-linearly. Nevertheless, the BLA+WGN nonlinearities appeared to be much stronger than those generated by BLA+8 $\mathrm{kHz}$. Strikingly, however, only WGN, but not pure-frequency tone, potentiated FrA pyramidal neurons when combined with the photo-stimulation of BLA-to-FrA inputs. Although this effect was analyzed no longer than $30 \mathrm{sec}$ after the end of auditory stimulation, it prompts the speculation that the additional non-linear dendritic depolarization gains control over $W G N$-related synapses. Indeed, compelling experimental evidence has demonstrated that non-linear interactions between compartmentalized streams of neural activity induce long-lasting changes in synaptic strength and intrinsic excitability (Dudman et al., 2007; Gambino et al., 2014; Jarsky et al., 2005; Larkum, 2013; McGaugh, 2013; Xu et al., 2012) that could have permanently affected the dynamics of the FrA membrane potential. 
The BLA presumably transfers information to the FrA that is relevant for fear learning (Lai et al., 2012; Nakayama et al., 2015). Surprisingly, inhibiting the activity of FrA neurons during CS-, or BLA neurons projecting to the FrA, attenuated freezing responses in response to CS+. However, this counterintuitive effect occurred only when $W G N$, but not $8 \mathrm{kHz}$ tone, was used as CS- during conditioning (protocol 1), suggesting that the BLA-mediated, non-linear integration of WGN in FrA neurons is critical for the acquisition of fear memory traces. This alteration of learning is unlikely to be the consequence of insufficient activation of the BLA. Instead, given the low number of BLA neurons expressing ArchT and their precise inhibition during CS-, an alternative explanation is that when $W G N$ is combined with the activation of BLA projecting axons, which is maximal between conditioning trials, it might promote the representation of sensory cues predicting threat (i.e. CS+, $8 \mathrm{kHz}$ ) within the FrA (Hall, 2002). This hypothesis is supported by the late modification of membrane potential fluctuations observed after the activation of BLA with WGN presentation, which occurred when an 8 $\mathrm{kHz}$ tone was presented during conditioning. In addition, we found that blocking the activity of BLA-to-FrA neurons transiently during CS- also increased freezing behaviors in response to CS- during recall. While these data are consistent with the role of BLA axons in the encoding of CS- as a safety predictor (Likhtik and Paz, 2015; Likhtik et al., 2014; Rogan et al., 2005; Sangha et al., 2013; Senn et al., 2014; Stujenske et al., 2014), they contrast with the effect of FrA inhibition, suggesting that this effect is perhaps dependent on the mPFC (Likhtik et al., 2014; Stujenske et al., 2014), possibly through specific individual axons targeting both the mPFC and the FrA (Mátyás et al., 2014).

BLA neurons send multiple projections to cortical and subcortical areas that have been shown to project also to the FrA. It is thus possible that the information is transmitted from the BLA to the FrA through an indirect pathway (Nakayama et al., 2015; Price, 2003). Here, we demonstrate that the expression of the genetically encoded calcium indicator GCaMP6 in BLA neurons makes it possible to monitor optically the activity of target-specific BLA axons during learning. Using this strategy in awake mice, we demonstrate for the first time that BLA-to-FrA axons are progressively recruited upon successive conditioning trials, thereby ruling out the indirect activation of the FrA circuit. First, we show that BLA-to-FrA axonal activity is never affected by the presentation of auditory cues alone. Our data contrast with previous work showing an increase in local field potential and unit activity in the BLA upon auditory stimulation (Collins and Paré, 
2000), and suggest instead the existence of a subpopulation of BLA neurons projecting specifically to the FrA that might play a specific role during the learning of emotion. Indeed, these axons are activated only after the first CS+/US pairing, and thus seem to transmit integrated information about the association itself (Nakayama et al., 2015). The level of activity of BLA-to-FrA axons was stronger between conditioning trials (i.e. during CS- presentation) and correlated with learning performance. This suggests a putative Hebbian-like frontal mechanism that could integrate any Gaussian noise that is contiguous to the maximal activation of BLA-to-FrA axons (Johansen et al., 2010, 2014; Larkum, 2013; Nakayama et al., 2015). While this mechanism would occur only if WGN is presented between conditioning trials (and thus used as CS-), it might eventually facilitate the recruitment of neurons into specific cue memory traces. In agreement, we found that auditory fear conditioning significantly decreased the number of $W G N$, but not $8 \mathrm{kHz}$ evoked local dendritic transients. Dendritic plateau potentials have been shown to regulate synaptic strength and synaptic plasticity (Cichon and Gan, 2015; Du et al., 2017; Gambino et al., 2014; Humeau and Lüthi, 2007; Palmer et al., 2014), which might subsequently facilitate the stabilization or pruning of synaptic inputs during learning (Holtmaat and Caroni, 2016; Li et al., 2017). In support of the latter, the level of fear learning has been shown to correlate with the percentage of spine elimination in FrA (Lai et al., 2012) which possibly explains the negative relation we observed during anesthesia between $W G N /(C S-)$-evoked subthreshold depolarizations and the strength of learning.

Collectively, our data reveal the specific properties of Gaussian noise in FrA during fear conditioning. The question arises as to the function or benefit of Gaussian noise in the BLA-to-FrA circuit during learning. Previous studies highlighted the critical function of the BLA in attention for learning (Laviolette et al., 2005; Roesch et al., 2010). Here, we show that the activation of BLA-to-FrA axons is independent of the nature of the CS presented. It thus seems unlikely that BLA-to-FrA axons convey the emotional valence of this association. In addition, the activation of BLA alone, while necessary, is not sufficient to trigger learning (Johansen et al., 2010, 2014). BLA neurons might signal to frontal circuits any new association independently of its valence, which might subsequently be assigned by the mPFC (Klavir et al., 2013; Likhtik and Paz, 2015). While acoustic noise is often viewed as a disturbing variable, it can enhance signal processing, facilitate sensory signaling and improve cognitive performance, notably in individuals with poor attention (McDonnell and Abbott, 2009; McDonnell and Ward, 2011; Stein et al., 2005). Therefore, 
bioRxiv preprint doi: https://doi.org/10.1101/569137; this version posted February 8, 2020. The copyright holder for this preprint (which was not certified by peer review) is the author/funder. All rights reserved. No reuse allowed without permission.

given that noise is abundant in the environment and communication of most mammals, it might facilitate learning in coordination with BLA-to-FrA inputs. 


\section{ACKNOWLEDGEMENTS}

We thank S. Deforges, E. Normand, and B. Darracq (Imetronic) for their technical expertise and support, and A. Holtmaat, P. Fossat, and S. Valerio (AquiNeuro) for their critical reading of our manuscript, and all the members of the Gambino laboratory for technical assistance and helpful discussions. We thank K. Deisseroth and Stanford University, E. Boyden and MIT, E.J. Kremmer and the IGMM BioCampus Montpellier, L.L. Looger and D. Kim from the GENIE project, and K. Svoboda at the Janelia Farm Research Campus (HHMI) for distributing viral vectors.

This project has received funding from (to FG): the European Research Council (ERC) under the European Union's Horizon 2020 research and innovation program (NEUROGOAL, grant agreement $n^{\circ}$ 677878), the FP7 Marie-Curie Career Integration program (grant agreement $n^{\circ} 631044$ ); the ANR JCJC (grant agreement $n^{\circ} 14$-CE13-001201), the University of Bordeaux (Initiative of Excellence senior chair 2014), the Laboratory of Excellence (LabEx) Brain grant 2015, and from the Region Nouvelle Aquitaine. NC is supported by a Marie Skłodowska-Curie individual fellowship under the European Union's Horizon 2020 research and innovation program (AXO-MATH; grant agreement $\left.\mathrm{n}^{\circ} 798326\right)$.

\section{AUTHOR CONTRIBUTIONS}

MA, EA, VK, NC performed the experiments. MA and FG conceived the studies and analyzed the data with the help of NC and EA. FG supervised the research and wrote the manuscript with help from MA, EA and NC.

\section{DECLARATION OF INTERESTS}

The authors declare no competing financial interests. 


\section{REFERENCES}

1. Antic, S.D., Zhou, W.-L., Moore, A.R., Short, S.M., and Ikonomu, K.D. (2010a). The decade of the dendritic NMDA spike. J. Neurosci. Res. 88, 2991-3001.

2. Antic, S.D., Zhou, W.-L., Moore, A.R., Short, S.M., and Ikonomu, K.D. (2010b). The decade of the dendritic NMDA spike. J. Neurosci. Res. 88, 2991-3001.

3. Basu, J., Zaremba, J.D., Cheung, S.K., Hitti, F.L., Zemelman, B. V., Losonczy, A., and Siegelbaum, S.A. (2016). Gating of hippocampal activity, plasticity, and memory by entorhinal cortex long-range inhibition. Science (80-. ). 351, aaa5694aaa5694.

4. Brandalise, F., Carta, S., Helmchen, F., Lisman, J., and Gerber, U. (2016). Dendritic NMDA spikes are necessary for timing-dependent associative LTP in CA3 pyramidal cells. Nat. Commun. 7, 13480.

5. Chen, X., Leischner, U., Rochefort, N.L., Nelken, I., and Konnerth, A. (2011). Functional mapping of single spines in cortical neurons in vivo. Nature 475, 501505.

6. Cichon, J., and Gan, W.-B. (2015). Branch-specific dendritic Ca(2+) spikes cause persistent synaptic plasticity. Nature 520, 180-185.

7. Collins, D.R., and Paré, D. (2000). Differential fear conditioning induces reciprocal changes in the sensory responses of lateral amygdala neurons to the $\mathrm{CS}(+)$ and CS(-). Learn. Mem. 7, 97-103.

8. Dejean, C., Courtin, J., Karalis, N., Chaudun, F., Wurtz, H.H., Bienvenu, T.C.M., and Herry, C. (2016). Prefrontal neuronal assemblies temporally control fear behaviour. Nature 535, 420-424.

9. Deneux, T., Kempf, A., Daret, A., Ponsot, E., and Bathellier, B. (2016). Temporal asymmetries in auditory coding and perception reflect multi-layered nonlinearities. Nat. Commun. 7, 12682.

10. Doron, M., Chindemi, G., Muller, E., Markram, H., and Segev, I. (2017). Timed Synaptic Inhibition Shapes NMDA Spikes, Influencing Local Dendritic Processing and Global I/O Properties of Cortical Neurons. Cell Rep. 21, 1550-1561.

11. Du, K., Wu, Y.-W., Lindroos, R., Liu, Y., Rózsa, B., Katona, G., Ding, J.B., and Kotaleski, J.H. (2017). Cell-type-specific inhibition of the dendritic plateau potential in striatal spiny projection neurons. Proc. Natl. Acad. Sci. U. S. A. 114.

12. Dudman, J.T., Tsay, D., and Siegelbaum, S.A. (2007). A role for synaptic inputs at distal dendrites: instructive signals for hippocampal long-term plasticity. Neuron $56,866-879$.

13. Gambino, F., Pagès, S., Kehayas, V., Baptista, D., Tatti, R., Carleton, A., and Holtmaat, A. (2014). Sensory-evoked LTP driven by dendritic plateau potentials in vivo. Nature 515.

14. Grosso, A., Cambiaghi, M., Concina, G., Sacco, T., and Sacchetti, B. (2015). Auditory cortex involvement in emotional learning and memory. Neuroscience 299, 45-55.

15. Hall, G. (2002). Associative Structures in Pavlovian and Instrumental Conditioning. In Stevens' Handbook of Experimental Psychology, (Hoboken, NJ, USA: John Wiley \& Sons, Inc.), p.

16. Hnasko, T.S., Perez, F.A., Scouras, A.D., Stoll, E.A., Gale, S.D., Luquet, S., Phillips, P.E.M., Kremer, E.J., and Palmiter, R.D. (2006). Cre recombinase-mediated restoration of nigrostriatal dopamine in dopamine-deficient mice reverses hypophagia and bradykinesia. Proc. Natl. Acad. Sci. U. S. A. 103, 8858-8863.

17. Holtmaat, A., and Caroni, P. (2016). Functional and structural underpinnings of neuronal assembly formation in learning. Nat. Neurosci. 19, 1553-1562. 
18. Hoover, W.B., and Vertes, R.P. (2007). Anatomical analysis of afferent projections to the medial prefrontal cortex in the rat. Brain Struct. Funct.

19. Humeau, Y., and Lüthi, A. (2007). Dendritic calcium spikes induce bi-directional synaptic plasticity in the lateral amygdala. Neuropharmacology 52, 234-243.

20. Jarsky, T., Roxin, A., Kath, W.L., and Spruston, N. (2005). Conditional dendritic spike propagation following distal synaptic activation of hippocampal CA1 pyramidal neurons. Nat. Neurosci. 8, 1667-1676.

21. Johansen, J.P., Hamanaka, H., Monfils, M.H., Behnia, R., Deisseroth, K., Blair, H.T., and LeDoux, J.E. (2010). Optical activation of lateral amygdala pyramidal cells instructs associative fear learning. Proc. Natl. Acad. Sci. 107, 12692-12697.

22. Johansen, J.P., Diaz-Mataix, L., Hamanaka, H., Ozawa, T., Ycu, E., Koivumaa, J., Kumar, A., Hou, M., Deisseroth, K., Boyden, E.S., et al. (2014). Hebbian and neuromodulatory mechanisms interact to trigger associative memory formation. Proc. Natl. Acad. Sci. 111, E5584-E5592.

23. Karalis, N., Dejean, C., Chaudun, F., Khoder, S., Rozeske, R.R., Wurtz, H., Bagur, S., Benchenane, K., Sirota, A., Courtin, J., et al. (2016). 4-Hz oscillations synchronize prefrontal?amygdala circuits during fear behavior. Nat. Neurosci. 19, 605-612.

24. Klavir, O., Genud-Gabai, R., and Paz, R. (2013). Functional connectivity between amygdala and cingulate cortex for adaptive aversive learning. Neuron 80, 12901300.

25. Klavir, O., Prigge, M., Sarel, A., Paz, R., and Yizhar, O. (2017). Manipulating fear associations via optogenetic modulation of amygdala inputs to prefrontal cortex. Nat. Neurosci. 20, 836-844.

26. Lai, C.S.W., Franke, T.F., and Gan, W.-B. (2012). Opposite effects of fear conditioning and extinction on dendritic spine remodelling. Nature 483, 87-91.

27. Larkum, M. (2013). A cellular mechanism for cortical associations: an organizing principle for the cerebral cortex. Trends Neurosci. 36, 141-151.

28. Laviolette, S.R., Lipski, W.J., and Grace, A.A. (2005). A subpopulation of neurons in the medial prefrontal cortex encodes emotional learning with burst and frequency codes through a dopamine $\mathrm{D} 4$ receptor-dependent basolateral amygdala input. J. Neurosci. 25, 6066-6075.

29. LeDoux, J.E. (2000). Emotion circuits in the brain. Annu. Rev. Neurosci. 23, 155184.

30. Li, W., Ma, L., Yang, G., and Gan, W.-B. (2017). REM sleep selectively prunes and maintains new synapses in development and learning. Nat. Neurosci. 20, 427-

615437.

31. Likhtik, E., and Paz, R. (2015). Amygdala-prefrontal interactions in (mal)adaptive learning. Trends Neurosci. 38, 158-166.

32. Likhtik, E., Stujenske, J.M., Topiwala, M.A., Harris, A.Z., and Gordon, J.A. (2014). Prefrontal entrainment of amygdala activity signals safety in learned fear and innate anxiety. Nat. Neurosci. 17, 106-113.

33. Major, G., Larkum, M.E., and Schiller, J. (2013). Active Properties of Neocortical Pyramidal Neuron Dendrites. Annu. Rev. Neurosci. 36, 1-24.

34. Martin-Cortecero, J., and Nuñez, A. (2016). Sensory responses in the medial prefrontal cortex of anesthetized rats. Implications for sensory processing. Neuroscience 339, 109-123.

35. Mátyás, F., Lee, J., Shin, H.-S.S., and Acsády, L. (2014). The fear circuit of the mouse forebrain: connections between the mediodorsal thalamus, frontal cortices and basolateral amygdala. Eur. J. Neurosci. 39, 1810-1823. 
36. McDonnell, M.D., and Abbott, D. (2009). What is stochastic resonance? Definitions, misconceptions, debates, and its relevance to biology. PLoS Comput. Biol. 5, e1000348.

37. McDonnell, M.D., and Ward, L.M. (2011). The benefits of noise in neural systems: bridging theory and experiment. Nat. Rev. Neurosci. 12, 415-425.

38. McGaugh, J.L. (2013). Making lasting memories: remembering the significant. Proc. Natl. Acad. Sci. U. S. A. 110 Suppl 2, 10402-10407.

39. Nakayama, D., Baraki, Z., Onoue, K., Ikegaya, Y., Matsuki, N., and Nomura, H. (2015). Frontal Association Cortex Is Engaged in Stimulus Integration during Associative Learning. Curr. Biol. 25, 117-123.

40. Palmer, L.M., Shai, A.S., Reeve, J.E., Anderson, H.L., Paulsen, O., and Larkum, M.E. (2014). NMDA spikes enhance action potential generation during sensory input.

41. Park, S., Lee, J., Park, K., Kim, J., Song, B., Hong, I., Kim, J., Lee, S., and Choi, S. (2016). Sound tuning of amygdala plasticity in auditory fear conditioning. Sci. Rep. 6, 31069.

42. Paxinos, G., and Watson, C. (2007). The rat brain in stereotaxic coordinates (Elsevier).

43. Price, J.L. (2003). Comparative aspects of amygdala connectivity. Ann. N. Y. Acad. Sci. $985,50-58$.

44. Rioult-Pedotti, M.S., Friedman, D., and Donoghue, J.P. (2000). Learning-induced LTP in neocortex. Science 290, 533-536.

45. Roesch, M.R., Calu, D.J., Esber, G.R., and Schoenbaum, G. (2010). Neural correlates of variations in event processing during learning in basolateral amygdala. J. Neurosci. 30, 2464-2471.

46. Rogan, M.T., Leon, K.S., Perez, D.L., and Kandel, E.R. (2005). Distinct neural signatures for safety and danger in the amygdala and striatum of the mouse. Neuron 46, 309-320.

47. Sacchetti, B., Baldi, E., Lorenzini, C.A., and Bucherelli, C. (2002). Differential contribution of some cortical sites to the formation of memory traces supporting fear conditioning. Exp. Brain Res. 146, 223-232.

48. Sangha, S., Chadick, J.Z., and Janak, P.H. (2013). Safety Encoding in the Basal Amygdala. J. Neurosci. 33, 3744-3751.

49. Senn, V., Wolff, S.B.E., Herry, C., Grenier, F., Ehrlich, I., Gründemann, J., Fadok, J.P., Müller, C., Letzkus, J.J., and Lüthi, A. (2014). Long-range connectivity defines behavioral specificity of amygdala neurons. Neuron 81, 428-437.

50. Sierra-Mercado, D., Padilla-Coreano, N., and Quirk, G.J. (2011). Dissociable roles of prelimbic and infralimbic cortices, ventral hippocampus, and basolateral amygdala in the expression and extinction of conditioned fear. Neuropsychopharmacology 36, 529-538.

51. Spruston, N., and Kath, W.L. (2004). Dendritic arithmetic. Nat. Neurosci. 7, 567569.

52. Stein, R.B., Gossen, E.R., and Jones, K.E. (2005). Neuronal variability: noise or part of the signal? Nat. Rev. Neurosci. 6, 389-397.

53. Stujenske, J.M., Likhtik, E., Topiwala, M.A., and Gordon, J.A. (2014). Fear and Safety Engage Competing Patterns of Theta-Gamma Coupling in the Basolateral Amygdala. Neuron 83, 919-933.

54. Tran-Van-Minh, A., Cazé, R.D., Abrahamsson, T., Cathala, L., Gutkin, B.S., and DiGregorio, D.A. (2015). Contribution of sublinear and supralinear dendritic integration to neuronal computations. Front. Cell. Neurosci. 9, 67. 
55. Uylings, H.B.M., Groenewegen, H.J., and Kolb, B. (2003). Do rats have a prefrontal cortex? Behav. Brain Res. 146, 3-17.

56. Vidal-Gonzalez, I., Vidal-Gonzalez, B., Rauch, S.L., and Quirk, G.J. (2006). Microstimulation reveals opposing influences of prelimbic and infralimbic cortex on the expression of conditioned fear. Learn. Mem. 13, 728-733.

57. Xu, N., Harnett, M.T., Williams, S.R., Huber, D., O'connor, D.H., Svoboda, K., and Magee, J.C. (2012). Nonlinear dendritic integration of sensory and motor input during an active sensing task. Nature 492, 247-251.

58. Zhang, S., Xu, M., Chang, W.-C., Ma, C., Hoang Do, J.P., Jeong, D., Lei, T., Fan, J.L., and Dan, Y. (2016). Organization of long-range inputs and outputs of frontal cortex for top-down control. Nat. Neurosci. 19, 1733-1742. 


\section{FIGURES AND LEGENDS}

Figure 1. Gaussian auditory stimulation depolarizes FrA pyramidal neurons

Figure 2. Gaussian auditory stimulation evokes more local dendritic events than pure frequency tones in anesthesia and awake

Figure 3. The FrA is engaged during fear learning when WGN is used as a CS-

Figure 4. The activation of BLA-to-FrA axons between conditioning trials predicts the level of auditory fear learning

Figure 5. The activation of BLA-to-FrA axons supports the non-linear integration of auditory tones

Figure 6. BLA-to-FrA projecting neurons are required for auditory fear learning when WGN is used as a CS-

Fig. S1. dAP5 application impacts spontaneous and auditory-evoked FrA membrane potential changes

705 Fig. S2. Auditory-evoked calcium events in dendritic tufts are similar between brain states

Fig. S3. Auditory tones do not affect locomotor activity.

Fig. S4. Effect of FrA opto-inhibition during conditioning on freezing responses and learning during recall

710 Fig. S5. Fear conditioning (protocol 1) specifically decreases WGN-induced local dendritic events

Fig. S6. Fear conditioning (protocol 1) occludes WGN-evoked somatic plateau potentials

Fig. S7. BLA-to-FrA axons do not encode the nature nor the valence of auditory 715 tones

Fig. S8. BLA-mediated synaptic inputs onto FrA L2/3 pyramidal neurons

Fig. S9. The activation of BLA-to-FrA axons supports the non-linear integration of auditory tones

Fig. S10. Statistical comparisons between all behavioral optogenetic experiments 


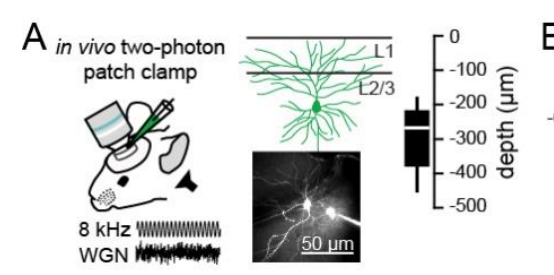

C
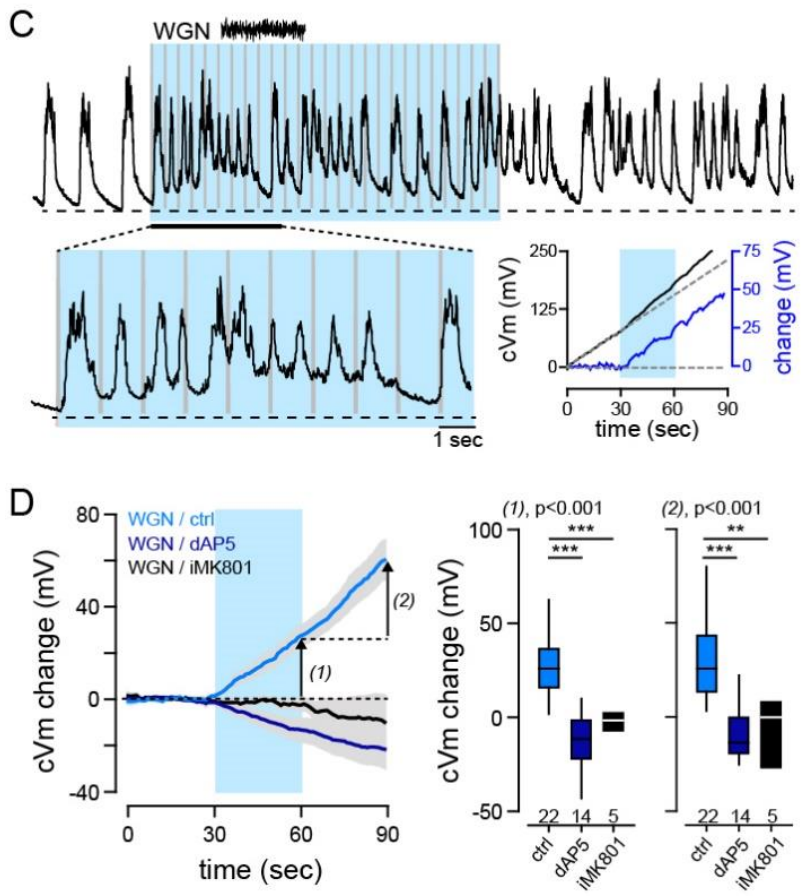
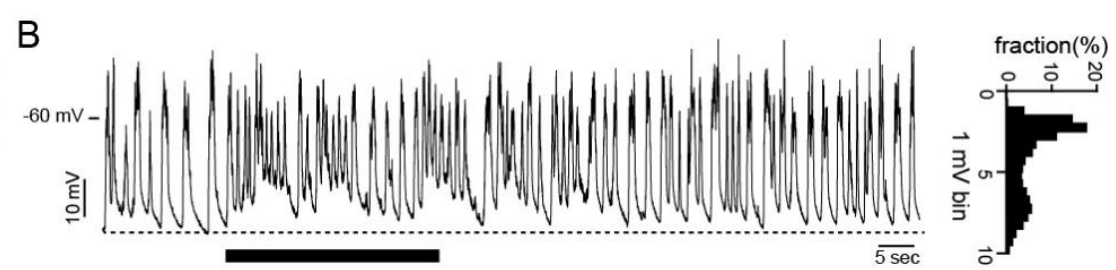

E $\quad 8 \mathrm{kHz}$ mumwmm
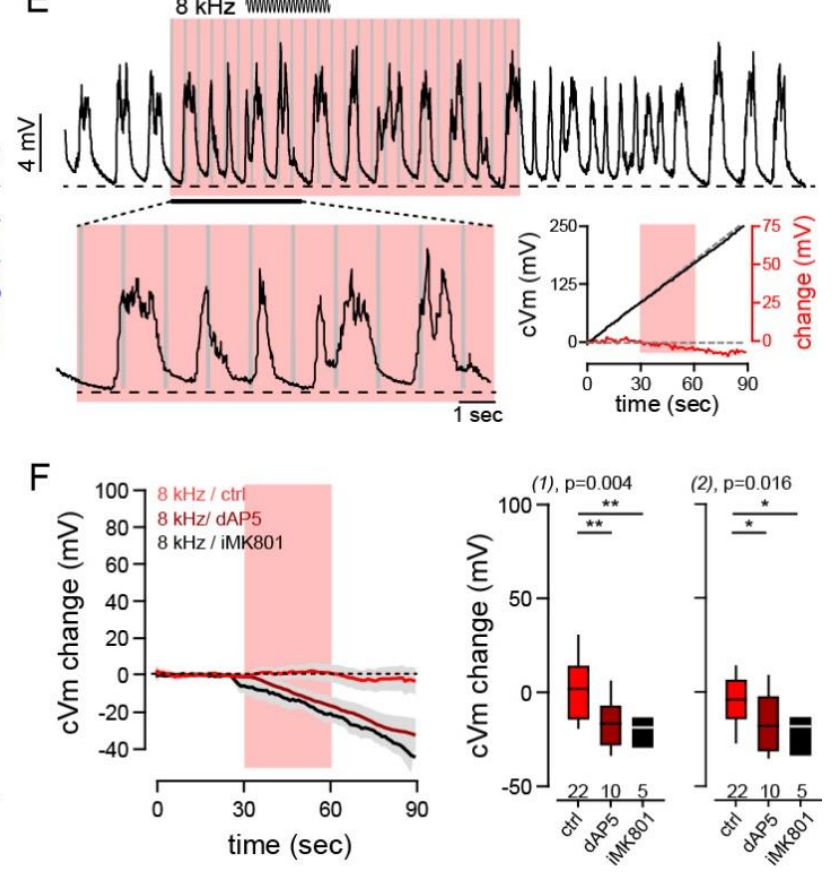

A) FrA pyramidal neuron membrane potential (Vm) was recorded from L2/3 FrA pyramidal under urethane anesthesia with $2 \mathrm{P}$ visual guidance. Depth of recorded cells is indicated.

B) Example of recorded FrA neuron showing typical spontaneous slow wave fluctuations.

Black bar indicates timing of Gaussian stimulation. Right, membrane potential probability histogram.

C) Example traces of postsynaptic membrane potential recorded from an individual FrA L2/3 pyramidal neuron upon white Gaussian noise (WGN) auditory stimulation (gray bars: 27 pips, $50 \mathrm{~ms}$ in duration, $0.9 \mathrm{~Hz}, 30 \mathrm{~s}$ ). Bottom right panels, auditory-evoked cVm changes that were calculated by subtracting the cumulative Vm (cVm, solid black line) by its linear regression during the baseline period (solid black line).

D) Left, averaged cVm change ( \pm sem) evoked by $W G N$, with or without the blockade of NMDARs (dAP5 or iMK801). Auditory stimulation is depicted by the blue bar; arrows, analysis time points 1 (end of stimulation) and 2 (30 sec later). Right, effect of dAP5 and iMK801 on cVm change at time points 1 (left, $\mathrm{p}<0.001$, one way anova) and 2 (right, $\mathrm{p}<0.001$, one way anova). Boxplots represent median and interquartile range (Ctrl, $\mathrm{n}=22$, dAP5, $\mathrm{n}=14$, iMK801, $\mathrm{n}=5$; ${ }^{* * *}, \mathrm{p}<0.001,{ }^{* *}, \mathrm{p}<0.01$, Holm-Sidak multiple comparisons).

E, F) Same representation as (C, D) but for pure $8 \mathrm{kHz}$-evoked cVm change (Ctrl, n=22, dAP5, n=10, iMK801, n=5; p=0.04 and 0.016, one way anova; ${ }^{* *}, \mathrm{p}<0.01,{ }^{*}, \mathrm{p}<0.05$, HolmSidak comparisons). The effect of both stimuli was tested on the same cell. 
A

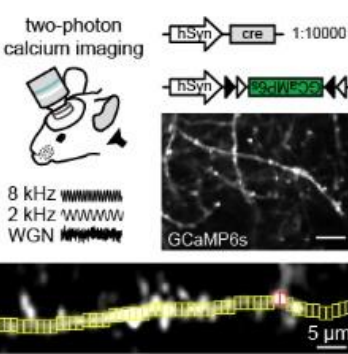

B
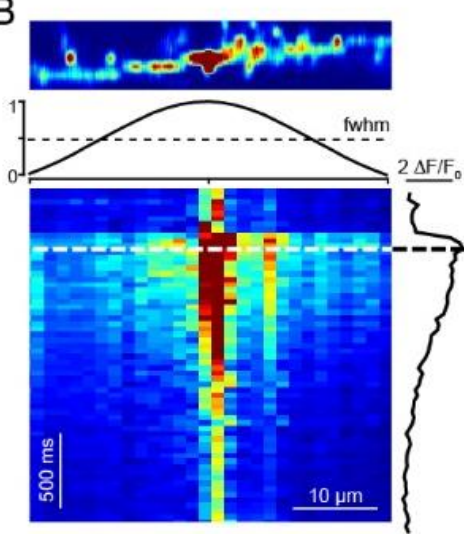

G

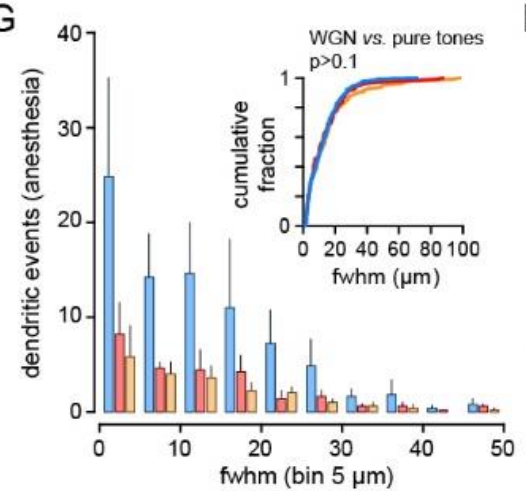

C
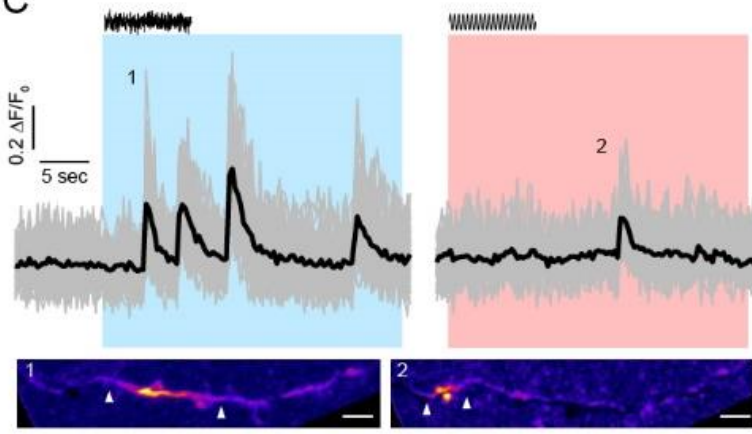

E
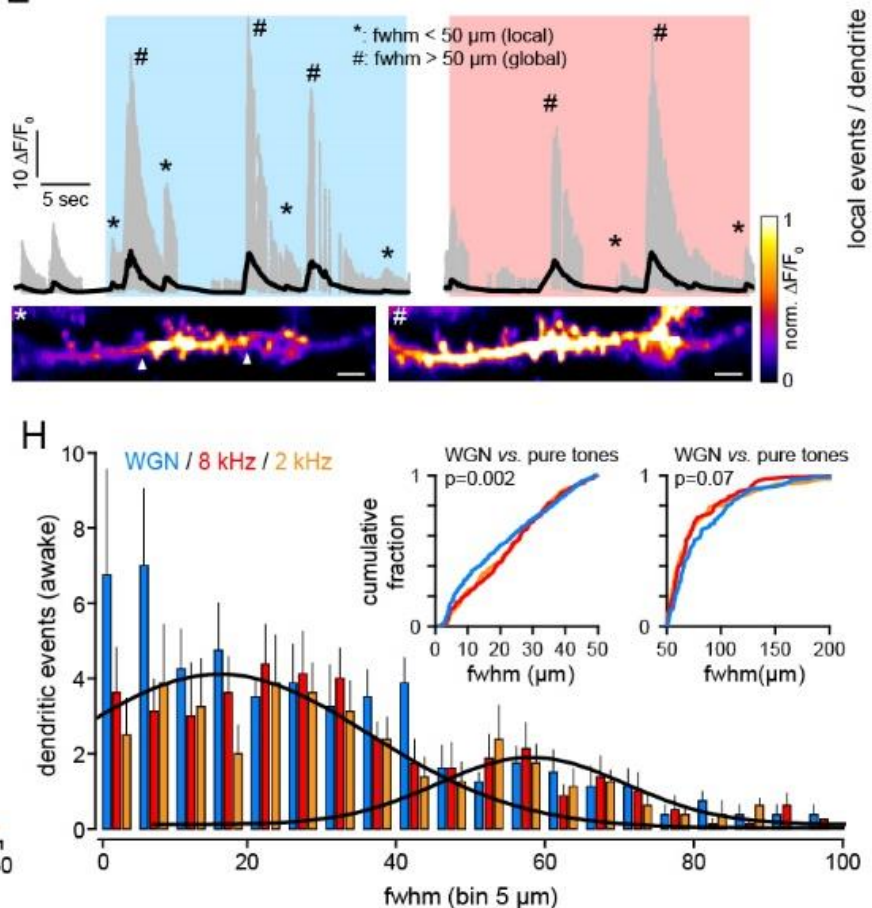

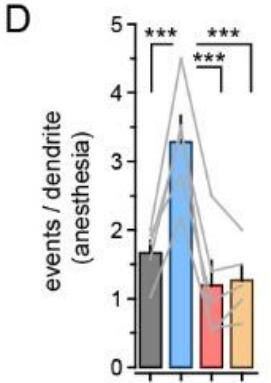

F
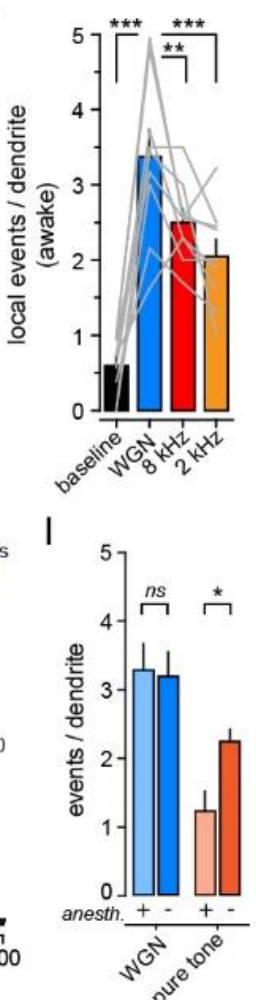

Figure 2. Gaussian auditory stimulation evokes more local dendritic events than pure frequency tones in anesthesia and awake

A) Top, experimental strategy. Mice were double-infected with AAV9.Flex.GcaMP6s and 1/10000 diluted AAV1.hSyn.Cre viruses resulting in sparse expression of GCaMP6s. Scale bar, $10 \mu \mathrm{m} . \mathrm{Ca}^{2+}$ events in individual dendrites were then imaged in L1 with 2P upon auditory stimulation ( $W G N, 8 \mathrm{kHz}$ and $2 \mathrm{kHz}$ ) in awake or anesthetized mice. Bottom, example of GCaMP6s fluorescence standard deviation image with ROIs in yellow. Red ROIs overlapped with another dendrite, and were hence excluded from the analysis.

B) The spread of $\mathrm{Ca}^{2+}$ events was quantified by calculating the full-width at half-max (fwhm, dashed line) of the normalized Gaussian fit at the time when the averaged $\Delta \mathrm{F} / \mathrm{F}_{0}$ was maximal.

C) Top, example of multiple $\mathrm{Ca}^{2+}$ transients $\left(\Delta \mathrm{F} / \mathrm{F}_{0}\right)$ recorded in a single dendrite in anesthetized mouse, during baseline and upon $W G N$ (blue) and $8 \mathrm{kHz}$ (red). Grey lines, $\Delta \mathrm{F} / \mathrm{F}_{0}$ measured from small ROIs (all ROIs superimposed). Black line, mean $\Delta \mathrm{F} / \mathrm{F}_{0}$ averaged over all ROIs, respectively. Bottom, examples of local dendritic $\mathrm{Ca}^{2+}$ events upon $W G N(1)$ and $8 \mathrm{kHz}(2)$. Scale bar, $5 \mu \mathrm{m}$.

D) The number of all $\mathrm{Ca}^{2+}$ events observed during baseline and upon auditory stimulations was averaged over 5 anesthetized mice $(\mathrm{p}<0.001$, one way repeated measures anova; *, $\mathrm{p}<0.05,{ }^{* * *}, \mathrm{p}<0.001$, Holm-Sidak multiple comparisons). Grey lines represent individual mice. 
E) Top, same representation as (C) but in awake mouse. Bottom, example of local $\left({ }^{*}\right)$ and global (\#) dendritic $\mathrm{Ca}^{2+}$ events. Scale bar, $5 \mu \mathrm{m}$.

F) Same presentation as in (D) but for local events from 9 awake mice $(\mathrm{p}<0.001$, one way repeated measures anova; $*, \mathrm{p}<0.05, * *, \mathrm{p}<0.01, * * *, \mathrm{p}<0.001$, Holm-Sidak multiple comparisons). Gray lines represent individual mice.

G, H) Distribution histogram of dendritic events fwhm upon WGN (blue), $8 \mathrm{kHz}$ (red) and $2 \mathrm{kHz}$ (orange) tone presentation, from anesthetized (G) and awake (H) mice. Inset, cumulative probability histograms of auditory-evoked events fwhm.

I) Dendritic events evoked by $W G N$ and pure tones $(8 \mathrm{kHz}$ and $2 \mathrm{kHz}$ tones pooled together) in anesthetized (+) and awake (-) mice. 
A

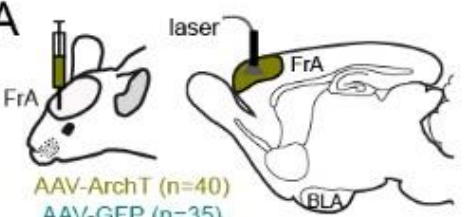
AAV-GFP $(n=35)$

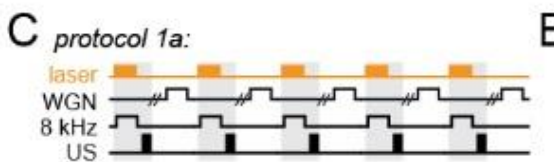

US

protocol 2a:

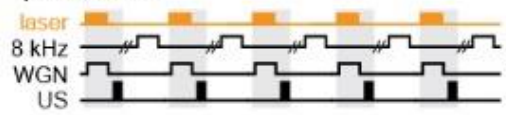

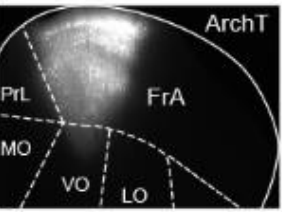

E

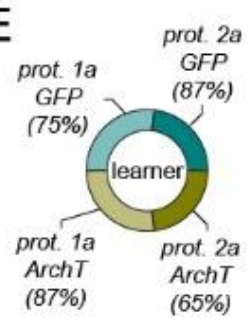

D

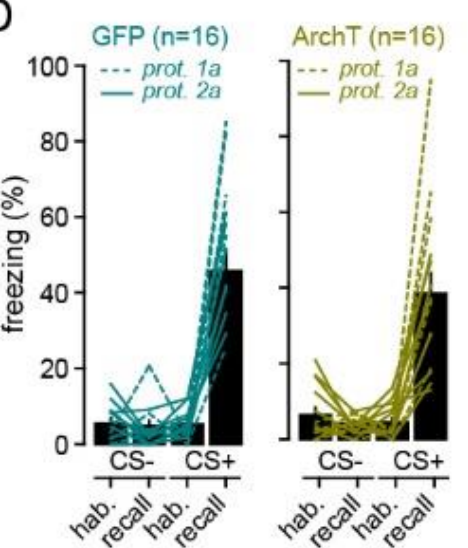

$\mathrm{F}$

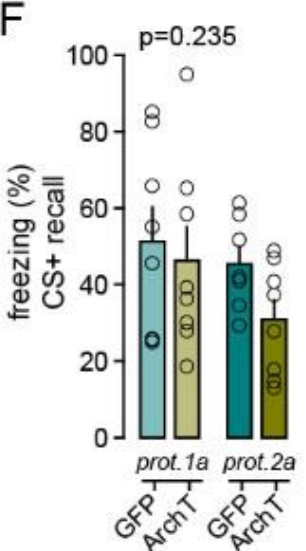

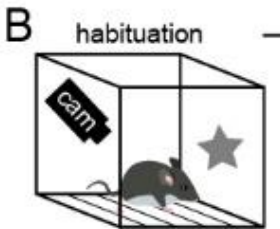
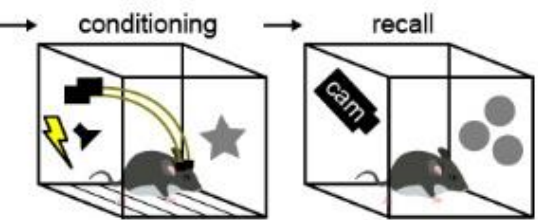

G
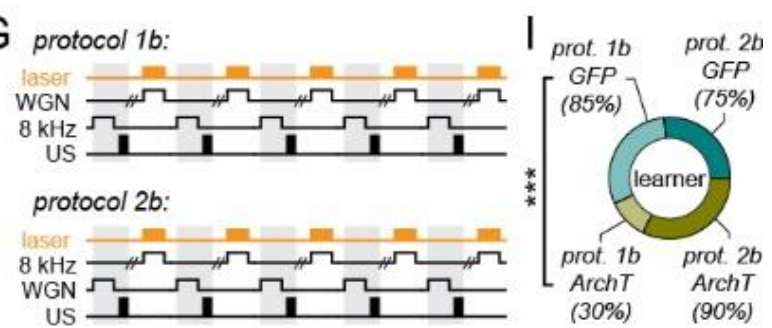

$\mathrm{H}$

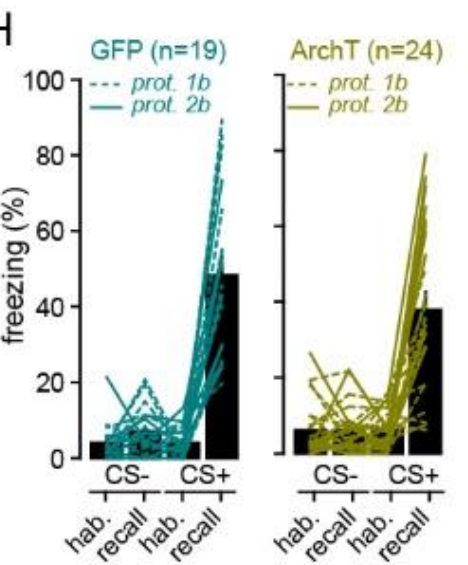

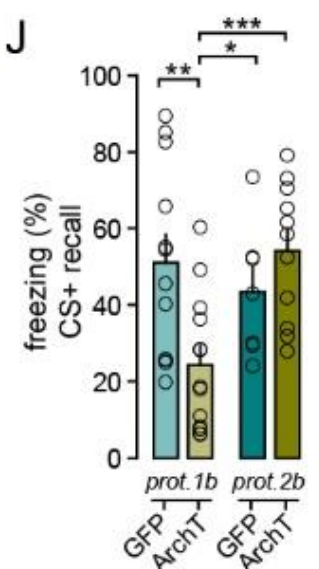

Figure 3. The FrA is engaged during fear learning when WGN is used as a CS-

A) Left, experimental strategy. Mice were infected with AAV9.CaMKII.ArchT $(n=40)$ or AAV9.CaMKII.eGFP for controls $(n=35)$ in FrA, and implanted bilaterally with optical fibers in FrA. Right, expression profiles of ArchT in the FrA (scale bar, $50 \mu \mathrm{m}$ ). PrL, prelimbic cortex; MO, VO, LO, medial, ventral and lateral orbitofrotal cortex respectively .

B) Timeline of auditory fear conditioning behavioral protocol, with habituation and fear conditioning done in one context and fear learning quantified $24 \mathrm{~h}$ later in a new context. C) Experimental fear conditioning protocols. FrA neurons expressing ArchT (or GFP) were photo-stimulated during the presentation of $\mathrm{CS}+(8 \mathrm{kHz}$ for protocol $1 \mathrm{a}$ or $W G N$ for protocol 1b). US, unconditional stimulus (footshock).

D) Effect of light during learning on freezing behaviors during recall as compared to habituation (hab.) in GFP-expressing mice (left; $n=16$ ) and ArchT-expressing mice (right, $\mathrm{n}=16$ ). Turquoise and khaki lines represent individual mice.

E) Fraction of mice that learned the cue-shocked association for each protocol.

F) Freezing behaviors quantified during CS+ in GFP- and ArchT-expressing mice ( $p=0.235$, anova). Circles, individual mice.

G-J) Same representation as (C-F) but for experiments with photo-stimulations delivered during CS-. 

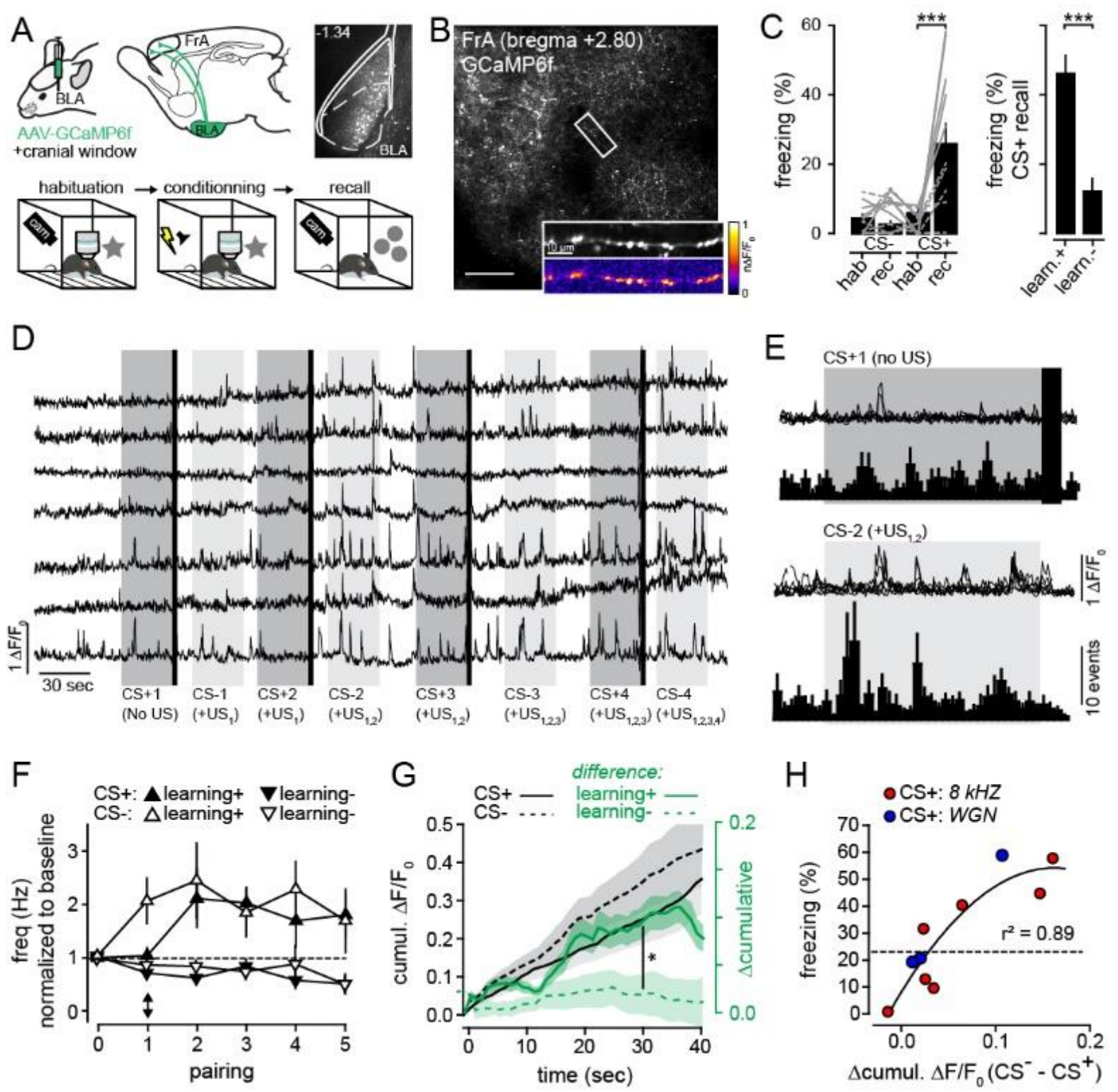

Figure 4. The activation of BLA-to-FrA axons between conditioning trials predicts the level of auditory fear learning

A) Top left, experimental protocol. BLA neurons were transfected with AAV1.Syn.GCaMP6f and their boutons were imaged in the superficial layer of the FrA. Top Right, expression profiles of GCaMP6f in the BLA. Bottom, GCaMP6f-expressing mice were fear conditioned under the 2P microscope (both protocols pooled together), and fear learning was quantified $24 \mathrm{~h}$ later in a new context.

B) GCaMP6f-expressing axons were imaged in the FrA of awake mice through a cranial window (scale bar, $200 \mu \mathrm{m}$ ). Inset, Example of $\mathrm{Ca}^{2+}$ transients in individual BLA-to-FrA axon.

815 C) Left, conditioning under the microscope induced robust fear behaviors ( $\mathrm{n}=10 ; \mathrm{p}=0.007$, two-way repeated measures anova): in contrast to CS-, CS+ increased fear freezing responses during recall as compared to habituation (hab.) (***, p $<0.001$, Holm-Sidak multiple comparisons). Gray solid lines, $\%$ of freezing of mice with learning index $>20 \%$ (learning+, $n=5$ ); gray dashed lines, $\%$ of freezing of mice with learning index $<20 \%$ 820 (learning-, $\mathrm{n}=5$ ). Right, freezing responses induced by $\mathrm{CS}+$ during recall for learners (learn. + ) and non-learners (learn. -) mice $(\mathrm{p}<0.001, t$-test $)$. 
D) Examples of $\mathrm{Ca}^{2+}$ transients $\left(\Delta \mathrm{F} / \mathrm{F}_{0}\right)$ from individual boutons recorded from one mouse upon consecutive CS+ / US pairings. Dark grey bars, CS+; light grey bars, CS-; black bars, footshock (US).

E) Examples of axonal $\mathrm{Ca}^{2+}$ transients $(t o p)$ and averaged histograms of distribution $( \pm$ sem) (bottom), for the first CS+ presentation (CS+1) and the second CS-presentation (CS2).

F) Frequency of axonal $\mathrm{Ca}^{2+}$ transients recorded during successive $\mathrm{CS}$. For the first $\mathrm{CS}+$, the activity of boutons was monitored in absence of footshock. Black arrow points to the difference between the first CS+ (before the first footshock) and the first CS- (after the first footshock). 0 corresponds to the baseline period before conditioning.

G) Cumulative $\Delta \mathrm{F} / \mathrm{F}_{0}$ averaged $( \pm$ sem) across all $\mathrm{CS}+$ (solid black lines, $\mathrm{n}=10$ ) and CS(dotted black lines, $\mathrm{n}=10$ ). Green lines, difference between $\mathrm{CS}+$ and $\mathrm{CS}$ - related axonal activity ( $\Delta$ cumulative: $\Delta \mathrm{F} / \mathrm{F}_{0 \mathrm{cs}-}-\Delta \mathrm{F} / \mathrm{F}_{0 \mathrm{cs}+}$ ) in mice that learned (learning+, solid green line, $\mathrm{n}=5$ ) or not (learning-, dotted green line, $\mathrm{n}=5$ ) the association. ${ }^{*}, \mathrm{p}=0.013, t$-test.

H) Relation between the $\Delta$ cumulative during conditioning and the $\%$ of freezing during recall for protocol 1 (blue, $\mathrm{CS}+: 8 \mathrm{kHz}$ ) and protocol 2 (red, CS+: $W G N$ ). Circles, mice. 

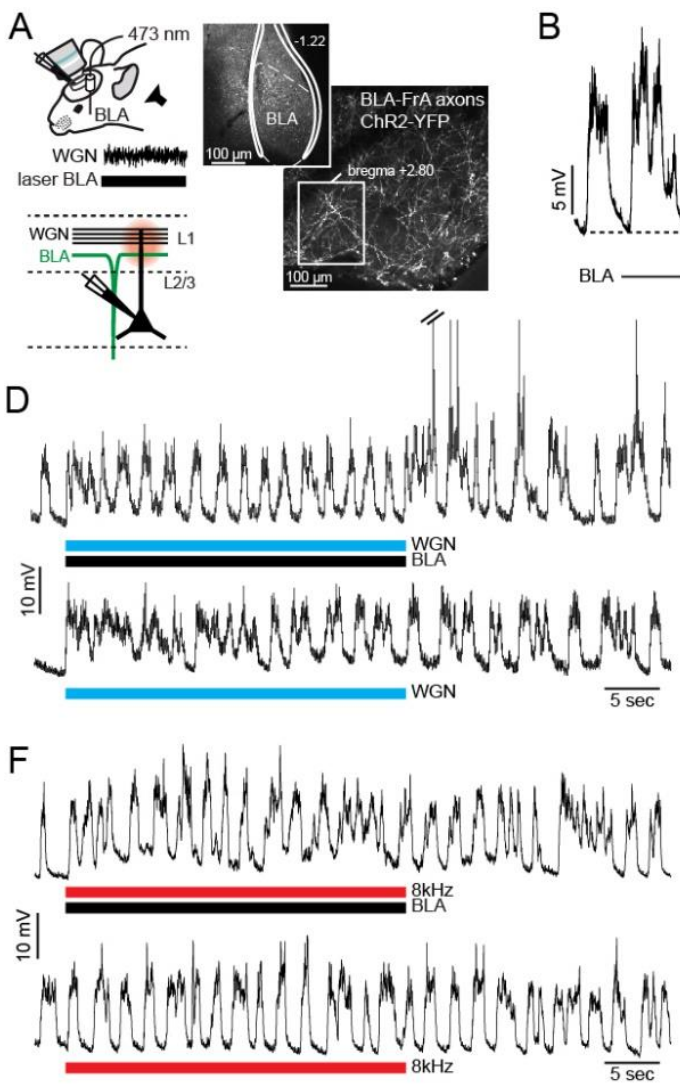

$\mathrm{E}$
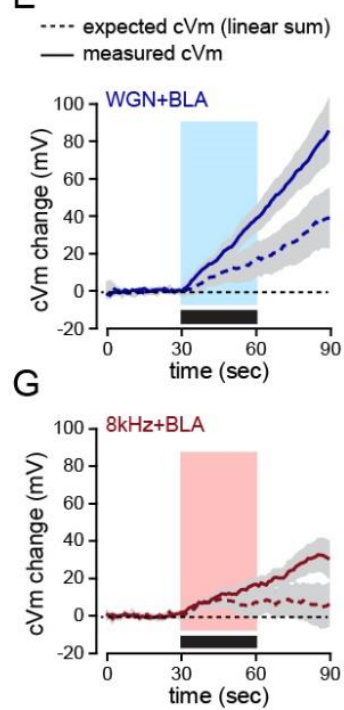

C

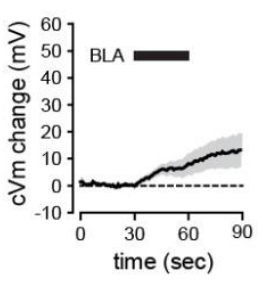

Figure 5. The activation of BLA-to-FrA axons supports the non-linear integration of auditory tones

A) Left, co-activation protocol. ChR2-expressing BLA neurons were photo-stimulated during auditory stimulation. Right, representative example of the ChR2-GFP expression profile in the mouse BLA and FrA (ChR2-expressing axons were imaged in superficial layer 1 with 2P microscopy before recording).

B) Single-cell example of depolarizations in FrA by the rhythmic photostimulation of BLA neurons (27 square stimulations, $50 \mathrm{~ms}, 0.9 \mathrm{~Hz}$ for $30 \mathrm{~s}$ ).

850 C) Averaged $\mathrm{cVm}$ change ( \pm sem) evoked by the photostimulation of BLA neurons. Photostimulation is depicted by the black bar.

D) Example of traces of postsynaptic membrane potential recorded from individual FrA L2/3 pyramidal neurons upon WGN paired (top) or not (bottom) with the photostimulation of ChR2-expressing BLA neurons. Black and blue bars below the traces indicate the duration of the stimulation.

E) Averaged $\mathrm{cVm}$ change ( \pm sem) observed upon paired stimulation (solid line) or expected from the arithmetic sum of individual depolarizations evoked by the stimulation of BLA or auditory tones alone (dotted line). Blue and black bars, auditory and BLA stimulations, respectively.

860 F, G) Same representation as in (D, E) but for $8 \mathrm{kHz}$.

H) Left, relation between observed and expected cVm. Circles, individual cells, squares, mean \pm sem. Right, Averaged cVm ( \pm sem). ${ }^{* *}, \mathrm{p}<0.01 ;{ }^{* * *}, \mathrm{p}<0.001 ;$ paired t-test. Gray lines indicate paired experiments.

I) Left, averaged observed cVm change ( \pm sem). Arrows, analysis time points 1 (end of stimulation) and 2 (30 sec later). Right, effect of photostimulation (+) on $W G N$ (dark blue) and $8 \mathrm{kHz}$ (black red)-evoked $\mathrm{cVm}$ at time points 1 and 2 . ${ }^{* *}, \mathrm{p}=0.003 ;{ }^{*}, \mathrm{p}=0.035 ;$ t-test. Circles, individual cells. 

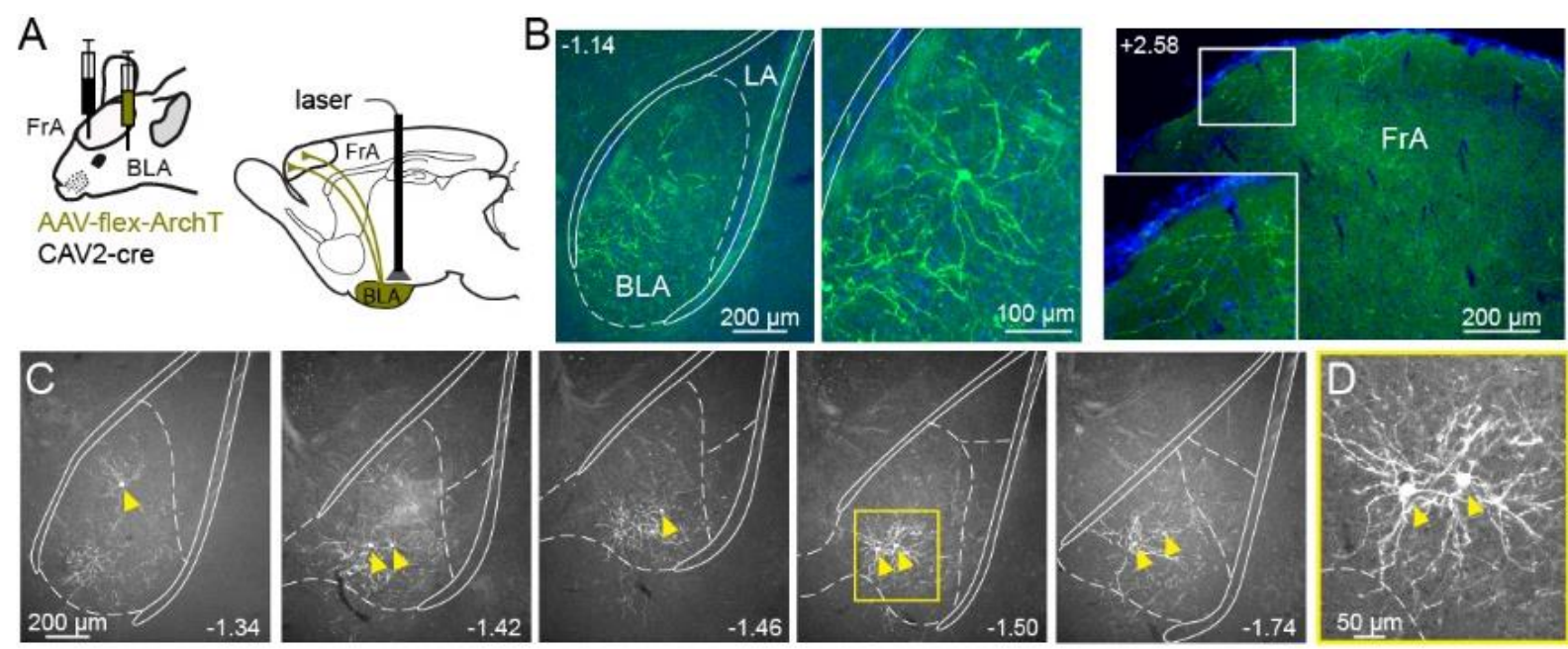

$E_{\text {protocol 1b: }}$

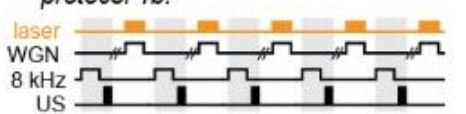

protocol $2 b$.

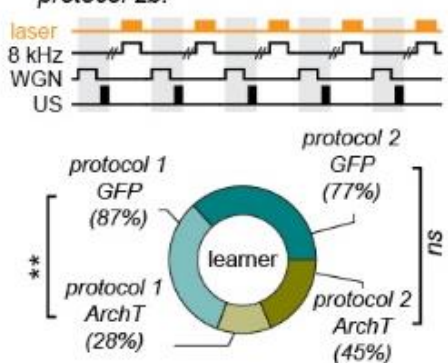

$\mathrm{F}$

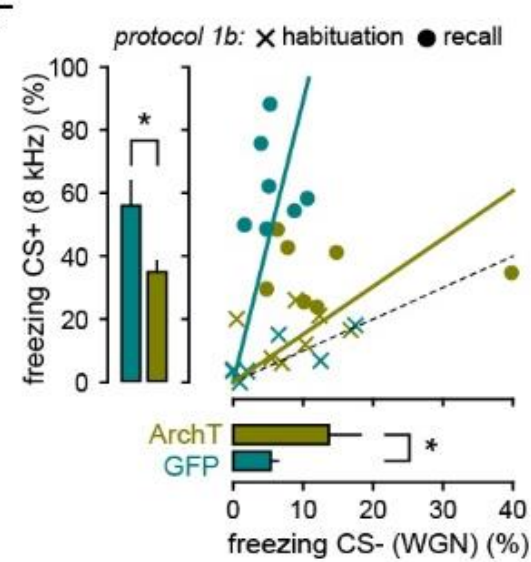

G

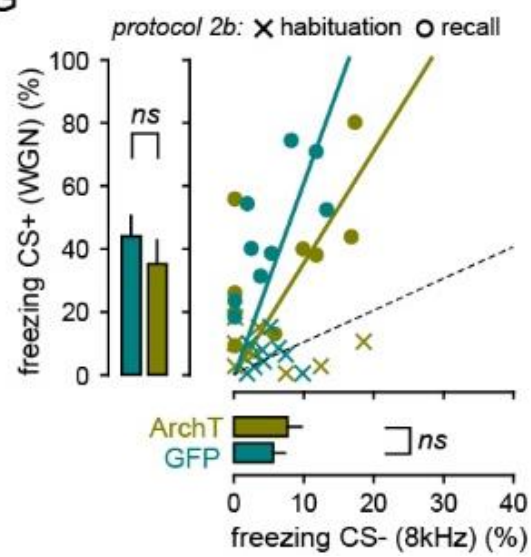

Figure 6. BLA-to-FrA projecting neurons are required for auditory fear learning when WGN is used as a CS-

A) Experimental strategy. Mice were infected with AAV9.CBA.Flex.ArchT-GFP (or AAV9.CAG.Flex.eGFP for controls) in BLA and CAV2.CMV.CRE in FrA, and chronically implanted bilaterally with optical fibers in BLA.

B) Example of expression profile of ArchT in BLA (left) and FrA (right). BLA, basolateral amygdala; LA, lateral amygdala.

C, D) Few neurons expressing ArchT were found throughout the entire BLA, but not the

LA.

E) Top, experimental fear conditioning protocols. FrA neurons expressing ArchT (or GFP) were photo-stimulated during the presentation of CS- (WGN for protocol 1b or $8 \mathrm{kHz}$ for protocol $2 \mathrm{~b}$ ). US, unconditional stimulus (footshock). Bottom, fraction of mice that learned the cue-shocked association for each protocol. ${ }^{* *}, \mathrm{p}<0.01$; ns, non-significant; Pearson $\chi^{2}$ test.

F) Effect of light on freezing responses during recall upon $8 \mathrm{kHz}$ (CS+ protocol 1b; GFP vs ArchT, p=0.037, t-test) and WGN (CS- protocol 1; GFP vs ArchT, p=0.04, t-test) presentation. The relation between $8 \mathrm{kHz}$ and WGN-evoked freezing during habituation (crosses) and recall (dotes) is also indicated.

890 G) Same presentation as in (F) but for WGN (CS+, protocol 2b) and $8 \mathrm{kHz}$ (CS-, protocol 2b). ns, p>0.05, t-test. 

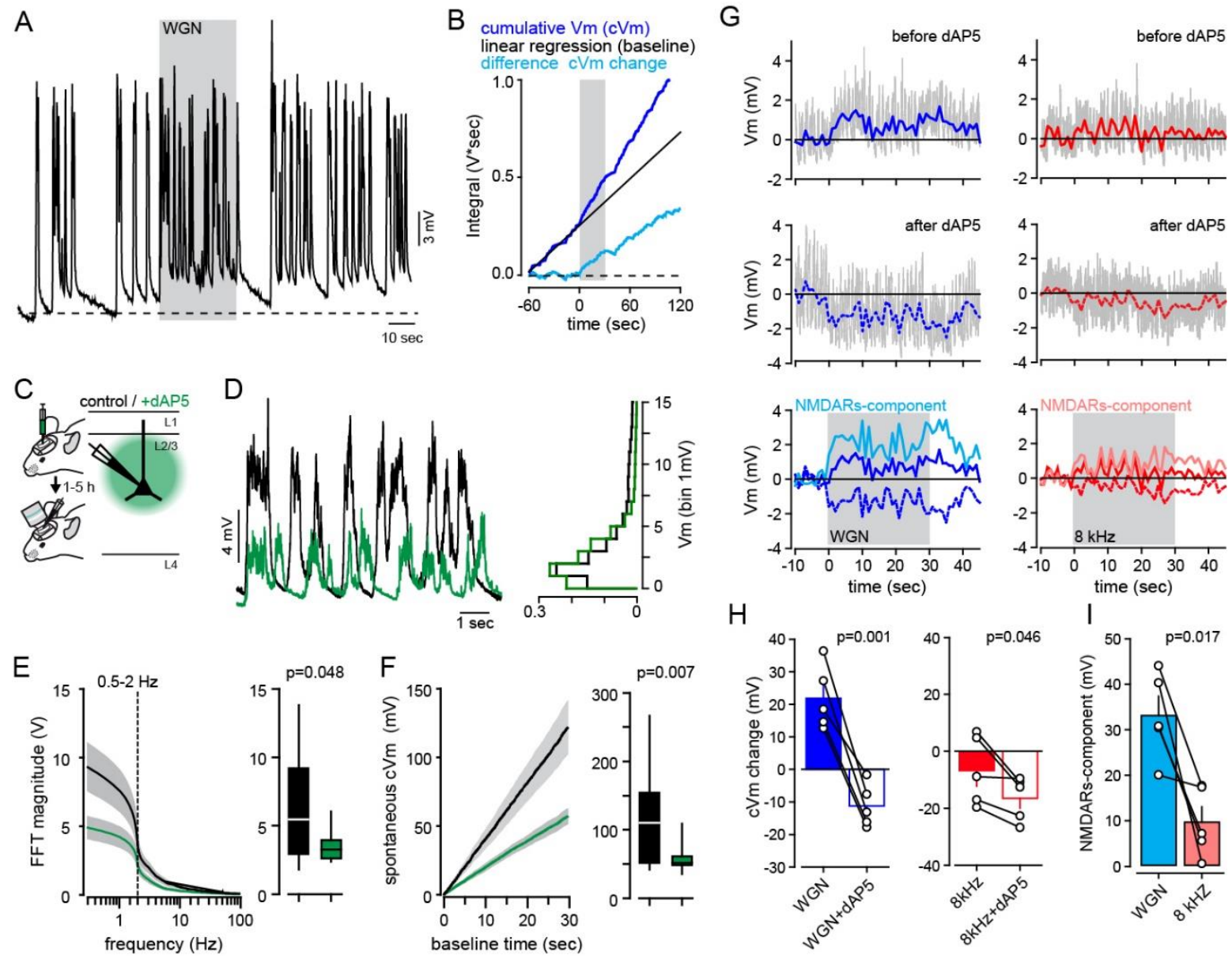

Fig. S1. dAP5 application impacts spontaneous and auditory-evoked FrA membrane potential changes

A) Single-cell example of typical spontaneous slow wave fluctuations.

B) WGN-evoked cVm change (light blue) is calculated by subtracting the cumulative Vm (cPSP, blue) by its linear regression during the baseline period (black line).

C) Experimental protocol: dAP5 $(1 \mu \mathrm{m})$ was applied through the dura and L2/3 pyramidal neurons were patched 1 to 5 hours later.

D) Left, examples of single-cell spontaneous membrane potential (Vm) fluctuations during anesthesia in controls (black) and upon dAP5 injection (green). Right, corresponding membrane potential probability histograms.

E) Left, average ( \pm sem) fast Fourier transform (FFT) of spontaneous membrane potentials in controls (black) and upon dAP5 injection (green). Right, blocking NMDARs significantly decreases the $0.5-2 \mathrm{~Hz}$ range ( $\mathrm{p}=0.048$, Mann-Whitney rank sum test). Box plot represents median and interquartile range (control, $n=22$; dAP5, $n=19$ ).

F) Left, average ( \pm sem) cumulative PSP (cPSP) of spontaneous membrane potentials in controls (black) and upon dAP5 injection (green). Right, blocking NMDARs significantly decreases $\mathrm{cVm}$ amplitude after $30 \mathrm{sec}(\mathrm{p}=0.007$, Mann-Whitney rank sum test). Box plot represents median and interquartile range (control, $n=22$; dAP5, $n=19$ ).

G) Single cell example of Vm fluctuation upon $W G N$ (left) and $8 \mathrm{kHz}$ (right) before and after dAP5 application. Colored bars represent averaged Vm over 1 sec time window. The NMDARs-mediated component is calculated by subtracting the averaged Vm before and after dAP5 application.

H, I) Average $\mathrm{cVm}( \pm$ sem $)$ change before and after dAP5 application. Circles and lines, paired cells. 
A

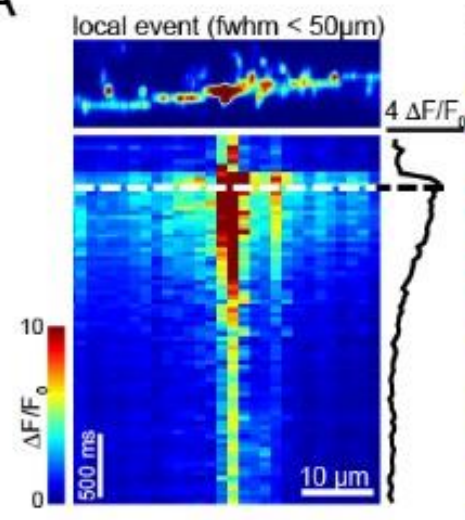

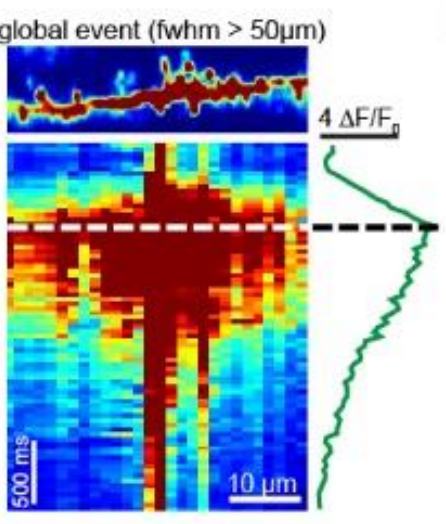

B

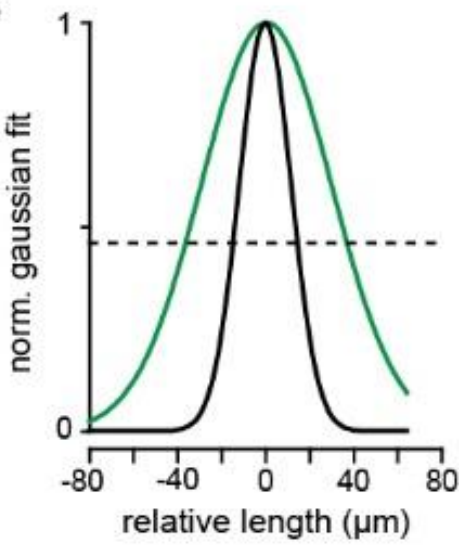

C

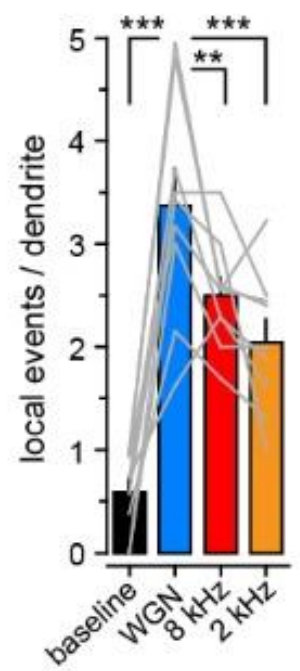

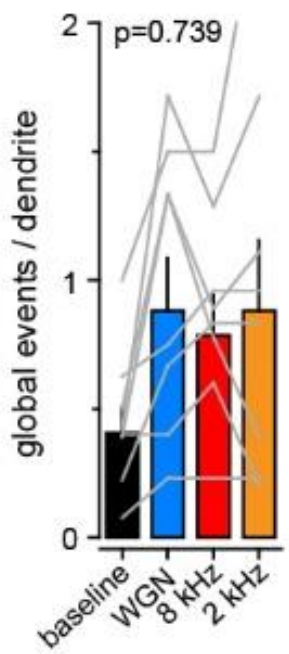

D anesthesia
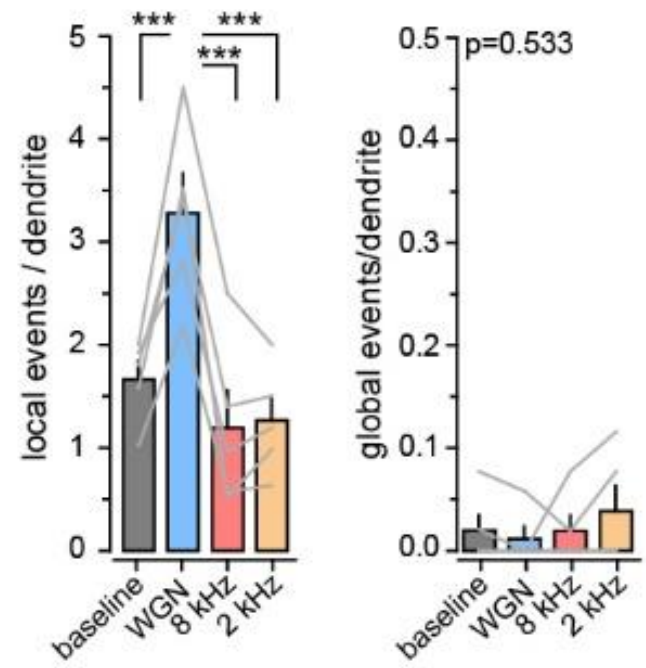

Fig. S2. Auditory-evoked calcium events in dendritic tufts are similar between brain states

A) Top, example of single-dendrite local (left) and global (right) $\mathrm{Ca}^{2+}$ events. Bottom, raster plot of $\Delta \mathrm{F} / \mathrm{F}_{0}$ for multiple ROIs along the dendrite represented above. Right, average $\Delta \mathrm{F} / \mathrm{F}_{0}$. Dashed line, time point of the maximum peak amplitude of averaged $\Delta \mathrm{F} / \mathrm{F}_{0}$.

B) Gaussian fits (normalized to max) of the local (black) and global (green) $\mathrm{Ca}^{2}$ events shown in (A).

C, D) number of local (left) and global (right) $\mathrm{Ca}^{2+}$ events observed during baseline and upon auditory stimulations in awake (E) and anesthetized (F) mice $(\mathrm{n}=9$ and 5, respectively). $\mathrm{p}<0.001$, one way repeated measures anova; ${ }^{* *}, \mathrm{p}<0.01,{ }^{* * *}, \mathrm{p}<0.001$, HolmSidak multiple comparisons). Grey lines represent individual mice. 
A

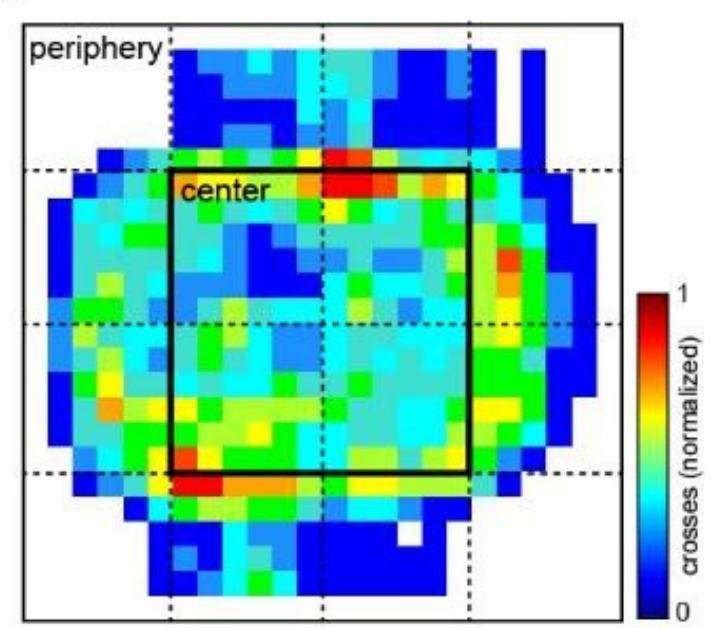

B

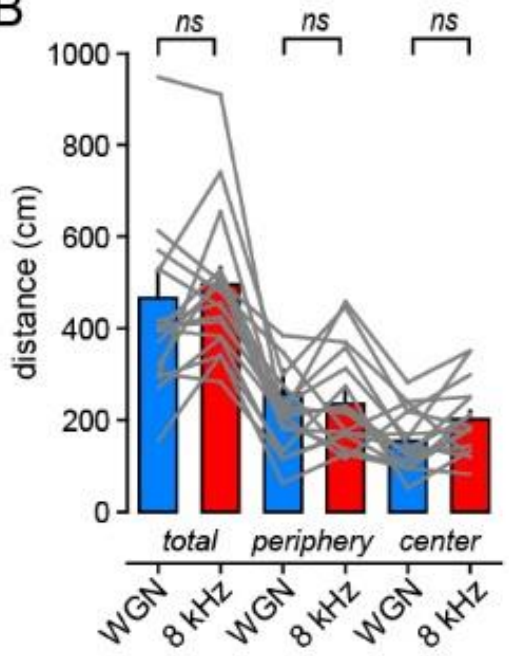

C

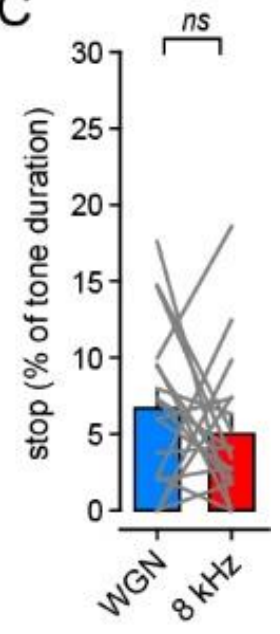

Fig. S3. Auditory tones do not affect locomotor activity.

A) Heatmap representation of mouse activity over 12 minutes. Each auditory tone was presented 4 times for 30 sec, every 10 to 60 seconds.

940 B) Averaged distance travelled (total, in the periphery, and in the center of the open field) upon $W G N$ and $8 \mathrm{kHz}$ tone presentation. Gray lines, individual mouse (n=17). ns, nonsignificant, post-hoc comparison after repeated measures anova.

C) Averaged stop duration upon tone presentation. Gray lines, individual mouse ( $\mathrm{n}=17)$. ns, paired t-test. 
A habituation 1-3

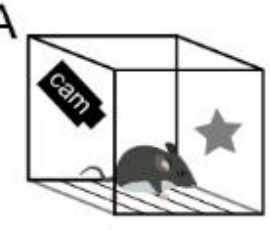

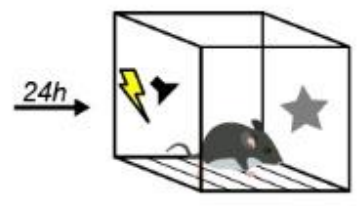

FC: fear conditioning pseudo-random pairings
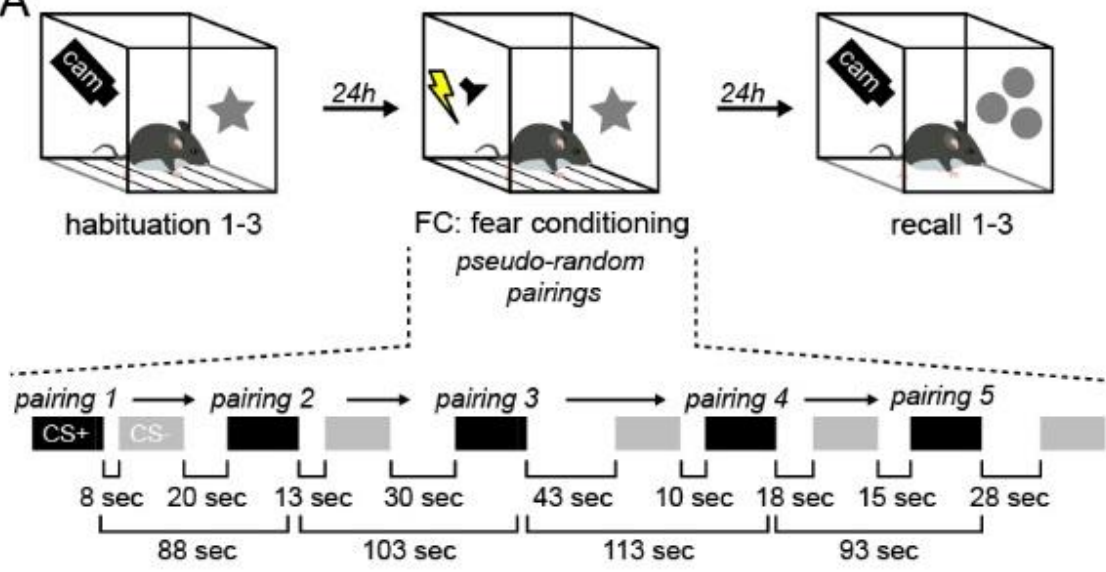

recall 1-3

B

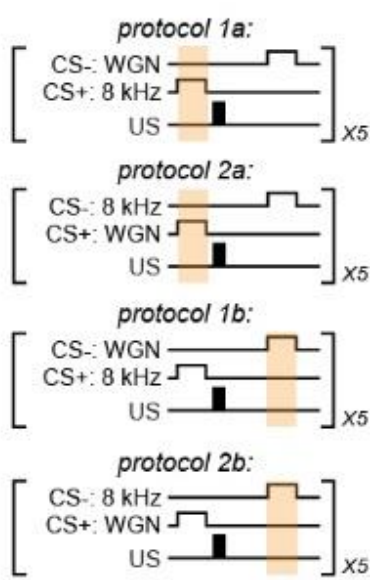

C
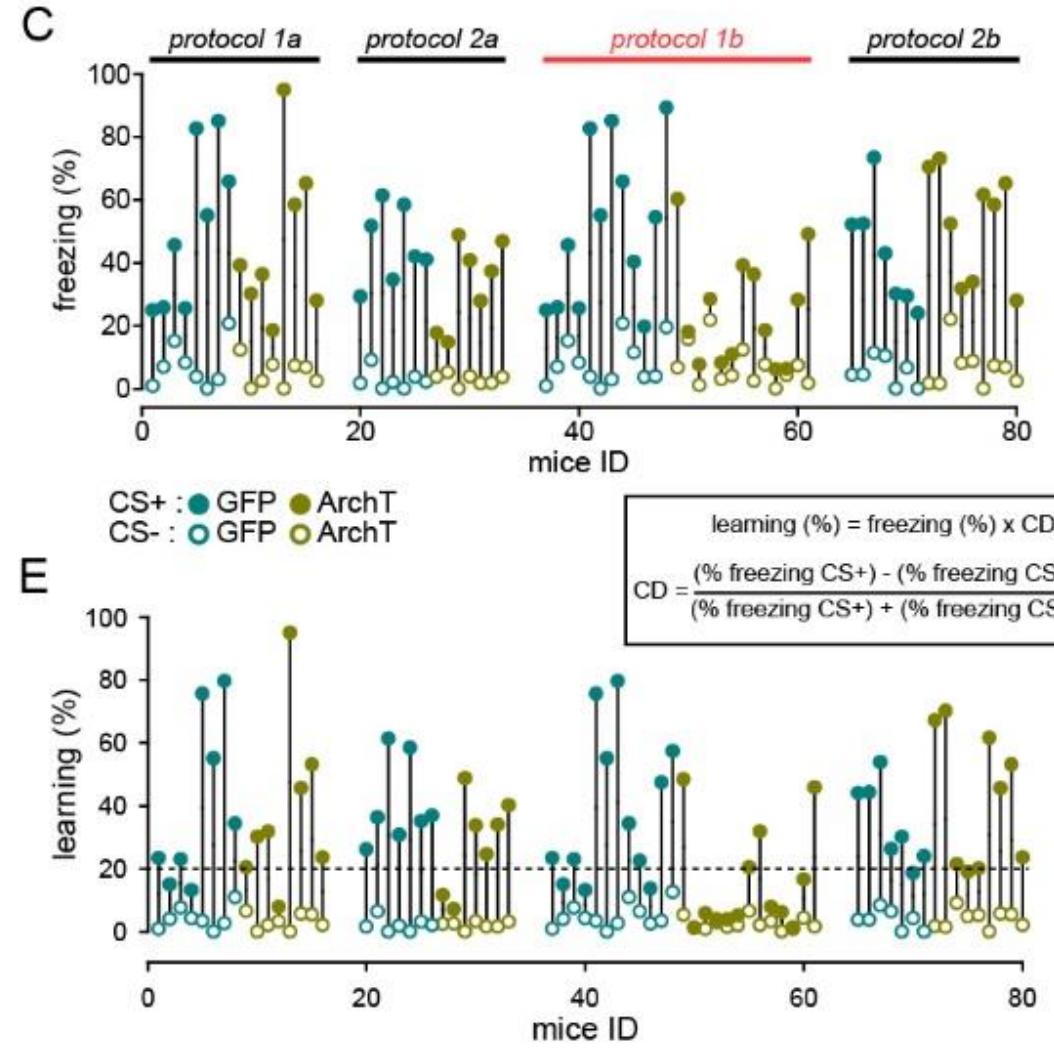

D

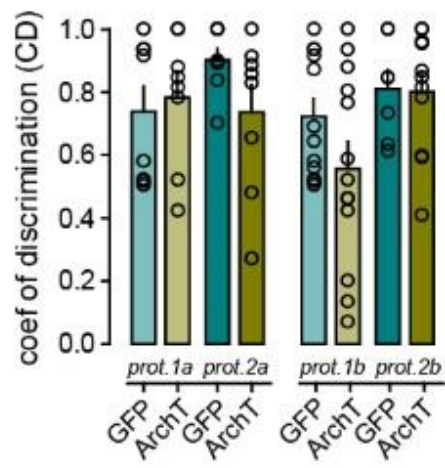

$\mathrm{F}$

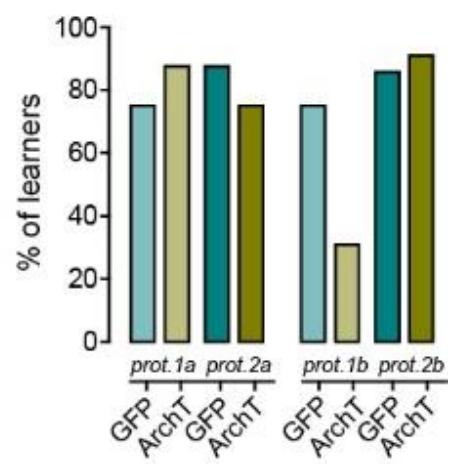

Fig. S4. Effect of FrA opto-inhibition during conditioning on freezing responses and learning during recall

A) Timeline of behavioral experiments. Random delays (between 10 and 60 seconds) between CS were used and one example is indicated below.

B) Fear conditioning protocols. FrA neurons expressing ArchT (or GFP) were photostimulated during the presentation of $\mathrm{CS}+(8 \mathrm{kHz}$ for protocol 1a or $W G N$ for protocol 2a) or CS- (WGN for protocol $1 \mathrm{~b}$ or $8 \mathrm{kHz}$ for protocol 2b). US, footshock. Photo-simulations (orange box) were given during CS+ (protocols $1 \mathrm{a}$ and $2 \mathrm{a}$ ) or CS - (protocols $1 \mathrm{~b}$ and $2 \mathrm{~b}$ ). C) Freezing responses during recall upon CS+ (filled circles) and CS- (open circles) in GFP (turquoise) and ArchT (khaki) expressing mice. Significant differences between GFP and ArchT-mice were seen only for protocol 1b (red).

D) Coefficient of discrimination (CD) was calculated as follows: $\mathrm{CD}=(\mathrm{CS}+\%$ freezing- $\mathrm{CS}$ $\%$ freezing) / (CS+ \% freezing+ CS- \% freezing). Circles, individual mice. 
E) Learning index was quantified for each CS by multiplying the \% of freezing in each condition by the corresponding coefficient of discrimination. Mice with a learning index below $20 \%$ (dotted line) were considered as non-learners.

F) fraction of mice that learned the cue-shock association (learning $>20 \%$ ). 

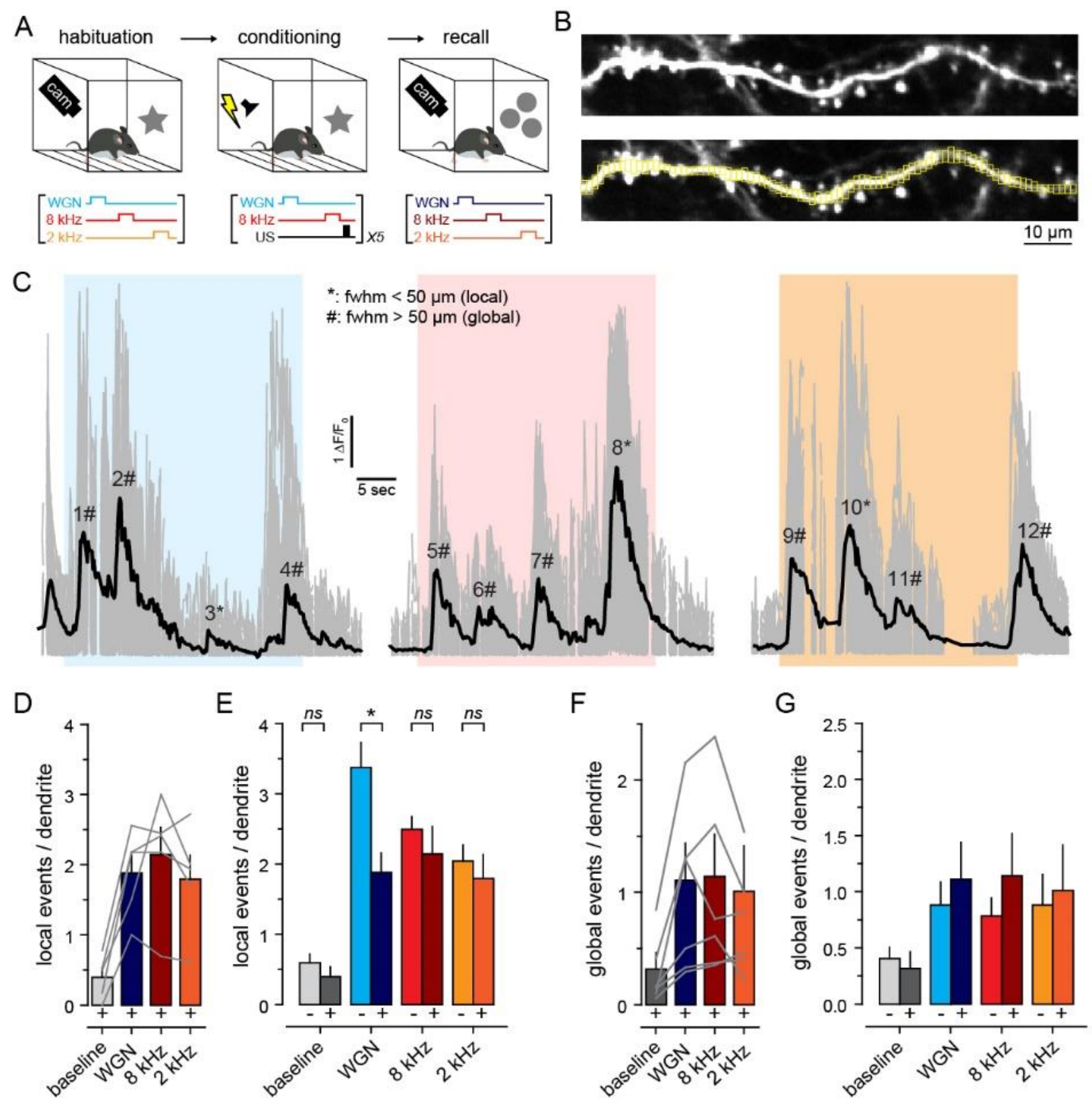

Fig. S5. Fear conditioning (protocol 1) specifically decreases WGN-induced local dendritic events

A) Top, Experimental protocol. GCAMP6s-expressing dendrites were imaged in awake mice before and after fear conditioning during presentation of $W G N, 8 \mathrm{kHz}$ and $2 \mathrm{kHz}$ tones. Bottom, fear conditioning protocol. Only $W G N$ and $8 \mathrm{kHz}$ were used during conditioning, while $2 \mathrm{kHz}$ served as a neutral tone that was only presented before and after conditioning.

B) Example of GCaMP6s fluorescence standard deviation projection (top) with ROIs in yellow (bottom).

C) Example of multiple $\mathrm{Ca}^{2+}$ transients $\left(\Delta \mathrm{F} / \mathrm{F}_{0}\right)$ recorded in a single dendrite, upon $W G N$ (blue), $8 \mathrm{kHz}$ (red) and $2 \mathrm{kHz}$ (orange). Grey lines, $\Delta \mathrm{F} / \mathrm{F}_{0}$ measured from small ROIs (all ROIs superimposed). Black line, mean $\Delta \mathrm{F} / \mathrm{F}_{0}$ averaged over all ROIs, respectively.

D) Examples of dendritic $\mathrm{Ca}^{2+}$ events upon $W G N$ (left, blue), $8 \mathrm{kHz}$ (middle, red), and $2 \mathrm{kHz}$ (right, orange). The spread of $\mathrm{Ca}^{2+}$ events was quantified by calculating the full-width at 
half-max (fwhm) of the normalized Gaussian fit (colored line) at the time when the averaged $\Delta \mathrm{F} / \mathrm{F}_{0}$ was maximal.

E, F) Number of local $\mathrm{Ca}^{2+}$ events per dendrite observed during baseline and upon auditory stimulations in awake mice before $(-, n=9)$ and after $(+, n=5)$ fear conditioning. The averaged number of local dendritic events per dendrite observed upon $W G N$ presentation in awake mice was significantly lower after fear learning ( $W G N-: 3.37 \pm 0.3$, $\mathrm{n}=9$ naive mice; $W G N+: 1.88 \pm 0.3, \mathrm{n}=5$ conditioned mice; $\mathrm{p}=0.025, t$-test).

G, H) Same presentation as in (E, F) but for global events. 

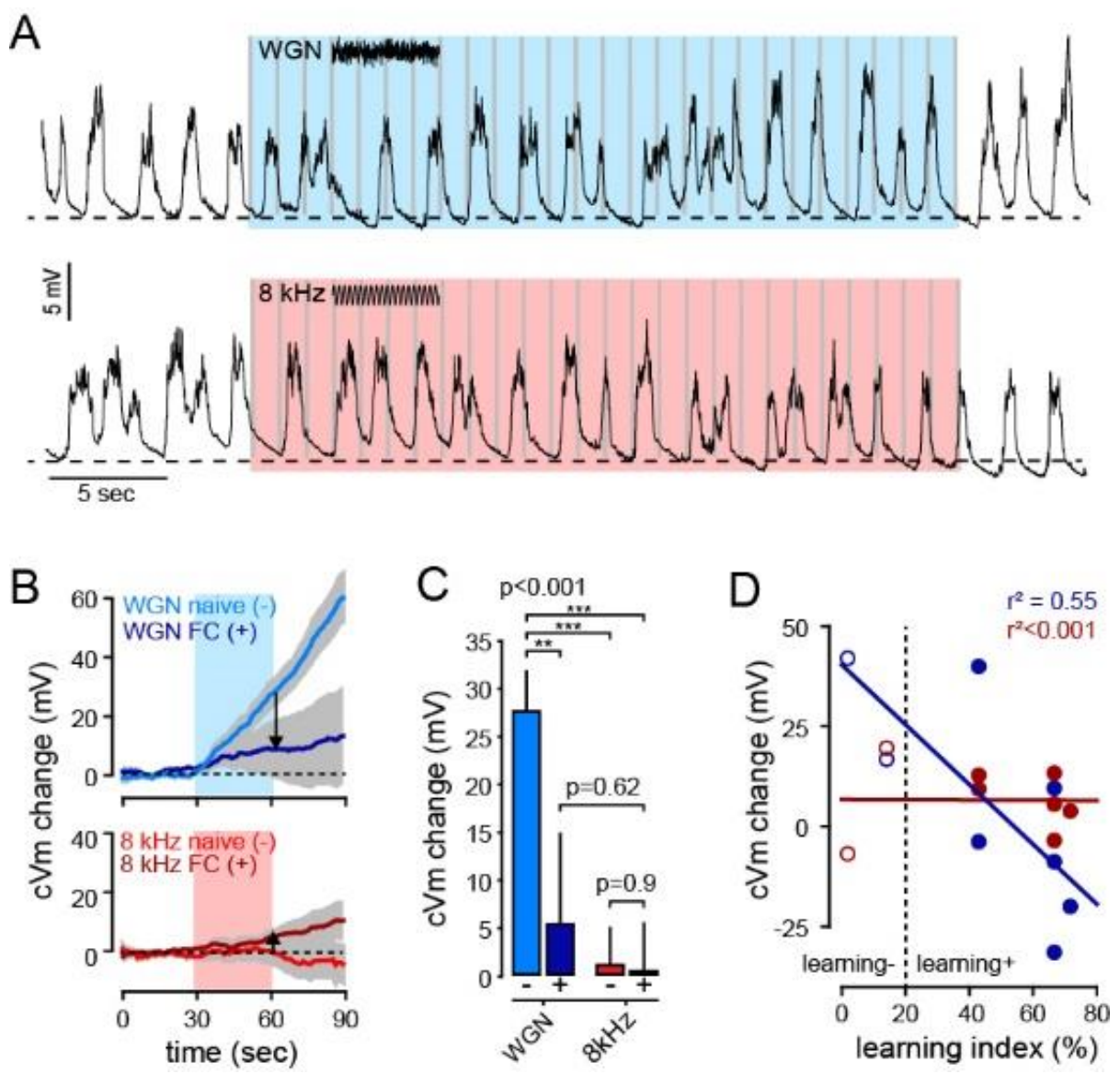

\section{Fig. S6. Fear conditioning (protocol 1) occludes WGN-evoked somatic plateau potentials}

995 A) Example traces of postsynaptic membrane potential recorded from an individual FrA pyramidal neuron upon $W G N$ (CS-, top) and $8 \mathrm{kHz}(\mathrm{CS}+$, bottom) auditory stimulation after fear conditioning. The effect of both stimuli was tested on the same cell.

B) Averaged cVm change ( \pm sem) evoked by $W G N$ (top) and $8 \mathrm{kHz}$ tones (bottom) before (naïve $(-)$ ) and after fear conditioning (FC (+)).

1000 C) Effect of WGN and pure 8kHz tone on cVm change before (-, n=22) and after learning $(+; \mathrm{n}=8)$. As compared to naive mice, $W G N$ failed to activate FrA pyramidal neurons in conditioned mice ( $W G N+: 5.4 \pm 9 \mathrm{mV}, \mathrm{n}=8 ; W G N-: 27.6 \pm 4 \mathrm{mV}, \mathrm{n}=22 ; \mathrm{p}=0.008)$. Thus, the difference between $W G N$ and $8 \mathrm{kHz}$-induced $\mathrm{cVm}$ change observed in naive mice (14 naive mice; $W G N$-: $27.6 \pm 4 \mathrm{mV}$; $8 \mathrm{kHz}$-: $1.1 \pm 4 \mathrm{mV}$; $\mathrm{n}=22$; $\mathrm{p}<0.001$; paired t-test) disappeared after fear conditioning ( 5 conditioned mice; $W G N+: 5.4 \pm 9 \mathrm{mV} ; 8 \mathrm{kHz}+: 0.5 \pm 5 \mathrm{mV}$; $\mathrm{n}=8$; $\mathrm{p}=0.698$; paired t-test). $\mathrm{p}<0.001$, one-way anova; ${ }^{* * *}, \mathrm{p}<0.001,{ }^{* *}, \mathrm{p}<0.01$, Holm-Sidak comparisons.

D) Relation between the learning index and $W G N$ (blue)- and $8 \mathrm{kHz}$ (red)-induced cVm change after conditioning. As opposed to $8 \mathrm{kHz}-\mathrm{cVm}$ change that was not different between behavioral performance $\left(\mathrm{r}^{2}<0.001\right), W G N$ induced stronger cVm change in low freezing mice indicating that WGN- somatic plateaus are negatively correlated with behavioral performance ( $\left.W G N: \mathrm{r}^{2}=0.55 ; 8 \mathrm{kHz}: \mathrm{r}^{2}<0.001\right)$. Open / filled circles, individual cells from non-learning / learning mice, respectively. 
A

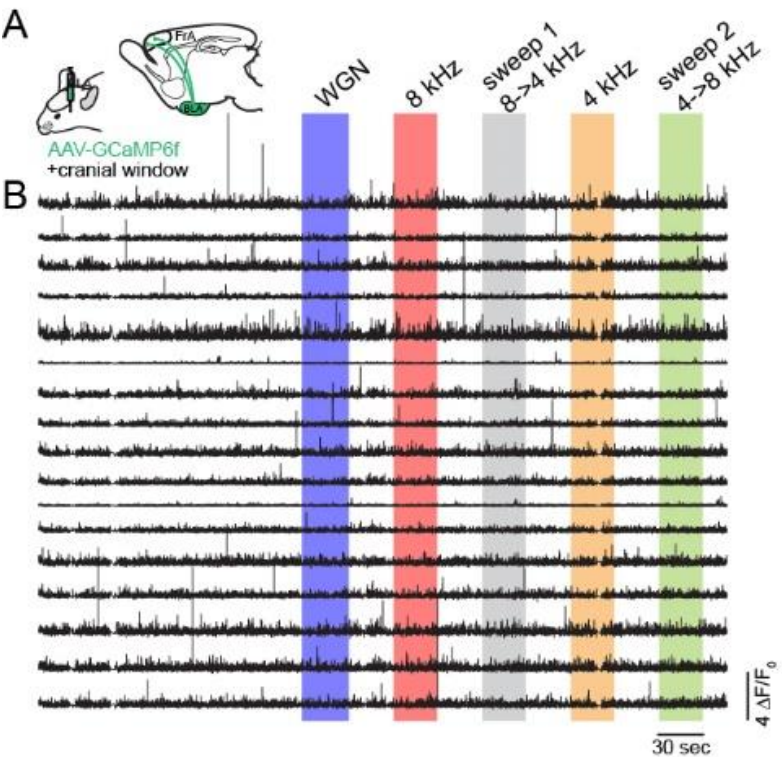

C

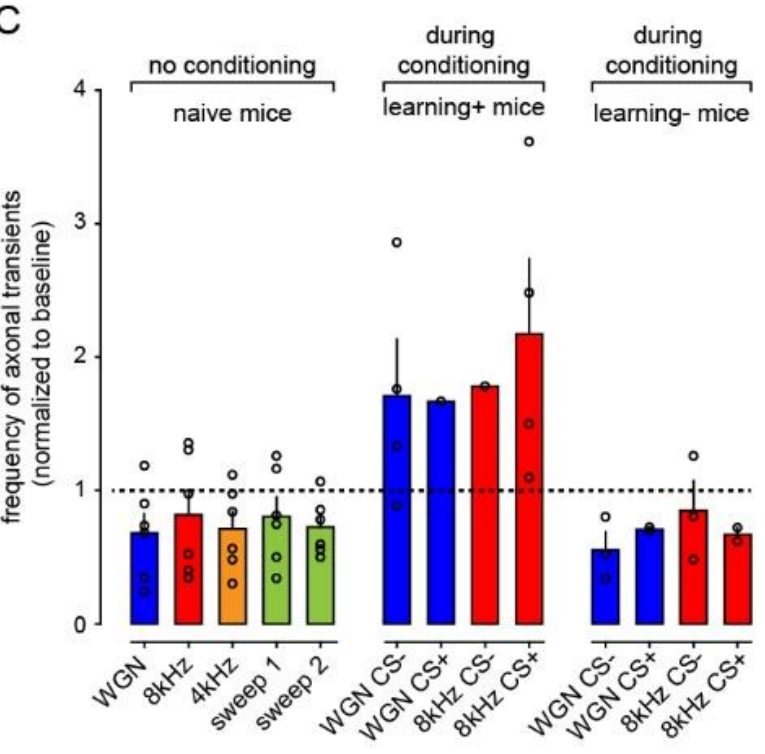

Fig. S7. BLA-to-FrA axons do not encode the nature nor the valence of auditory tones

A) Experimental protocol. BLA neurons were transfected with AAV1.Syn.GCaMP6f. GCaMP6f-expressing axons were imaged in the FrA of awake mice through a cranial window.

B) Examples of $\mathrm{Ca}^{2+}$ transients $\left(\Delta \mathrm{F} / \mathrm{F}_{0}\right)$ from individual boutons recorded from one mouse upon different auditory tones.

C) Frequency of axonal $\mathrm{Ca}^{2+}$ transients (normalized to baseline) recorded in naïve mice (no conditioning, $\mathrm{n}=6$ mice) and during auditory fear conditioning ( $\mathrm{n}=10$ mice) with protocol 1 (CS+: $8 \mathrm{kHz}, \mathrm{CS}$-: $W G N, \mathrm{n}=7$ ) and protocol 2 (CS+: $W G N, \mathrm{CS}$-: $8 \mathrm{kHz}, \mathrm{n}=3)$. 
A

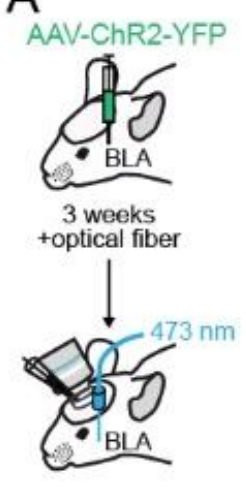

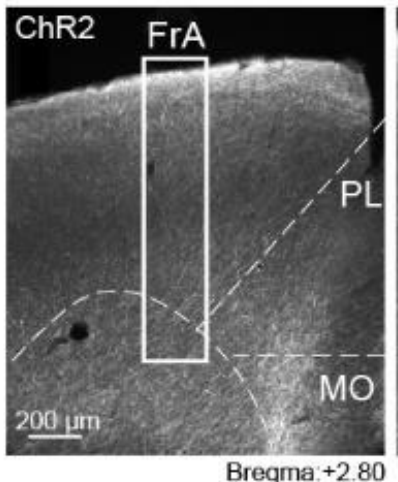

normalized intensity

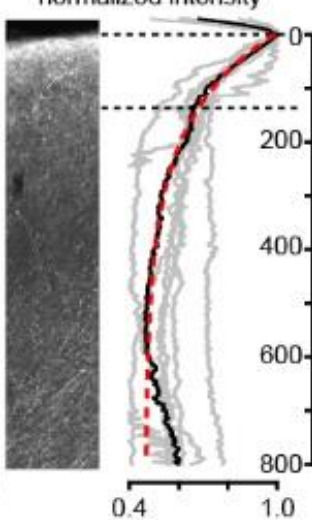

B

BLA-FrA axons

$\rightarrow$ ChR2-YFP.
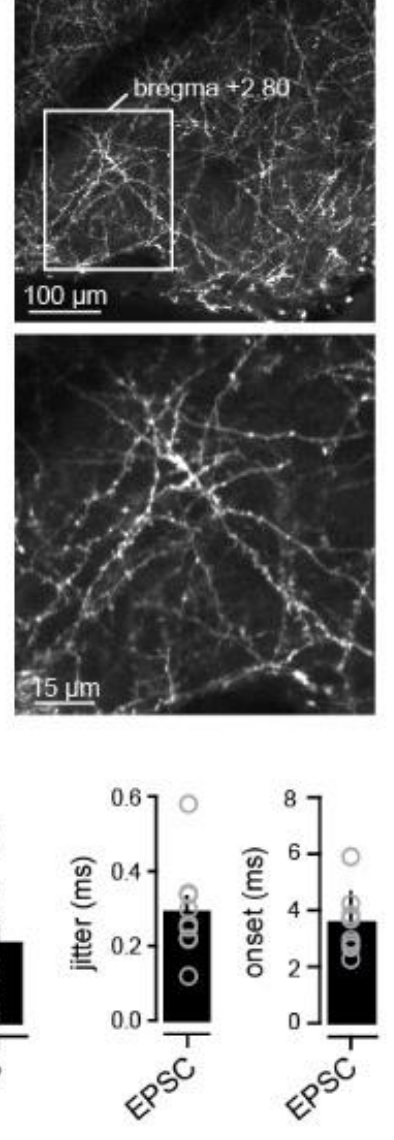

$G$

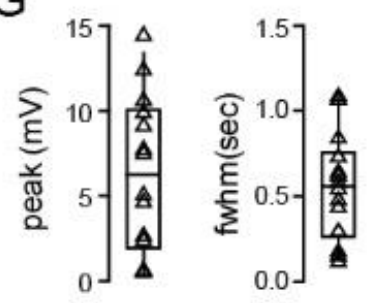

$\varepsilon^{S^{\circ}}$
F

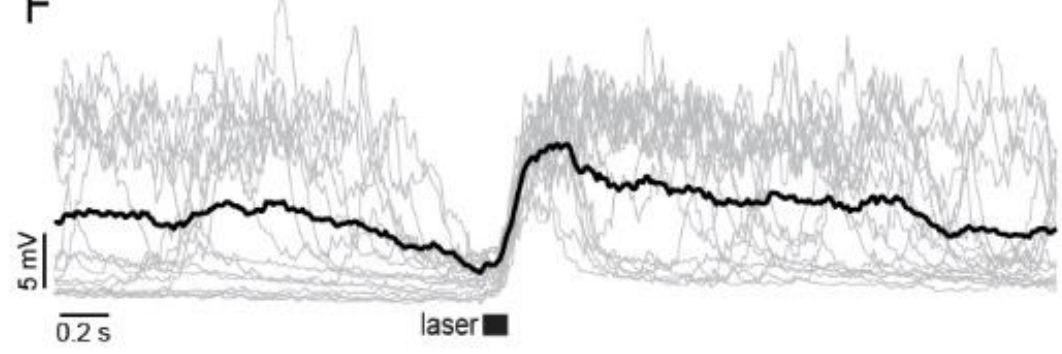

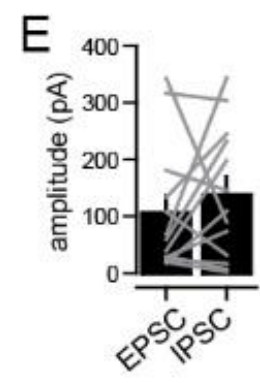

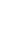

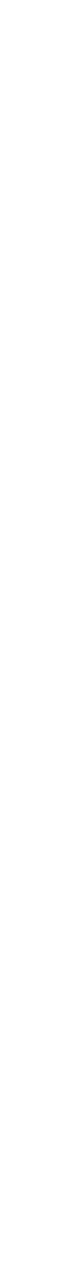

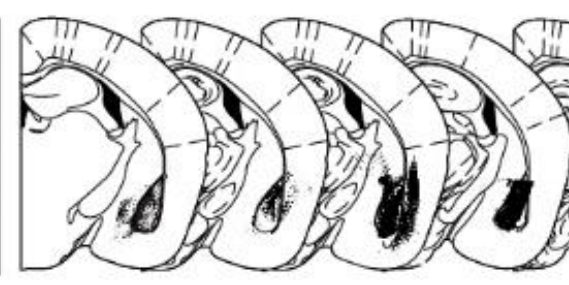
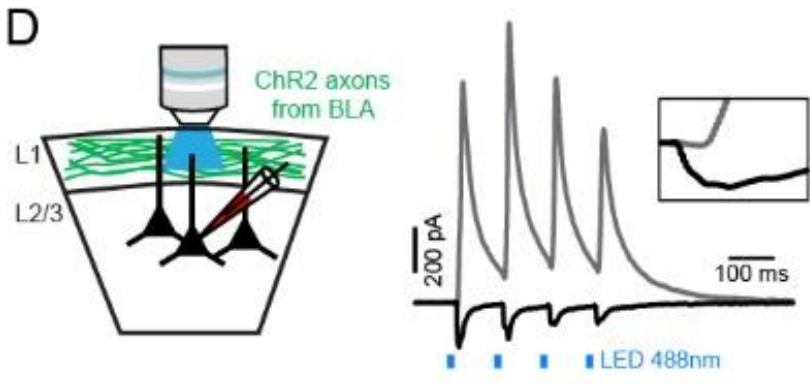

\section{Fig. S8. BLA-mediated synaptic inputs onto FrA L2/3 pyramidal neurons}

A) Experimental strategy. Left, BLA neurons were transfected with AAV9-CamKIIahChR2-eYFP, and ChR2-expressing BLA neurons were then photo-stimulated in vivo with a DPSSL $(\lambda=473 \mathrm{~nm})$ through an implanted optical fiber. Middle, example of a cortical coronal slice with ChR2-YFP fluorescence in the FrA. PL, prelimbic cortex; MO, medial orbital cortex. Right, fluorescence intensity profiles measured in the FrA (white box) of 6 different animals in which injections targeted BLA.

B) Examples of ChR2-expressing axons imaged in superficial layer 1 with 2P microscopy. C) Left, representative example of the ChR2-YFP expression profile in the mouse BLA. Right, Coronal diagrams of the brains from 6 mice showing the expression profiles (in black) of ChR2-YFP. Diagrams were adapted from the Paxinos atlas.

D) Schematic of the slice experiment. BLA-to-FrA axons expressing ChR2 were photoactivated through the objective. Right, gray and black lines, representative examples of 
excitatory post-synaptic current (EPSCs) and feed-forward inhibitory post-synaptic currents (IPSCs) respectively evoked on L2/3 pyramidal neurons with an ultrahigh power $488 \mathrm{~nm}$ LED.

E) Amplitude (left) and jitter (right) of light-evoked EPSCs. Gray lines indicate pairs. Open circles indicate individual cells.

F) Single-cell examples of depolarizations in FrA neuron evoked by the photostimulation of BLA neurons. Gray lines, single trials; black line, average of 20 trials.

G) Peak amplitude (left) and fwhm (right) of evoked PSP. Triangles, cells.
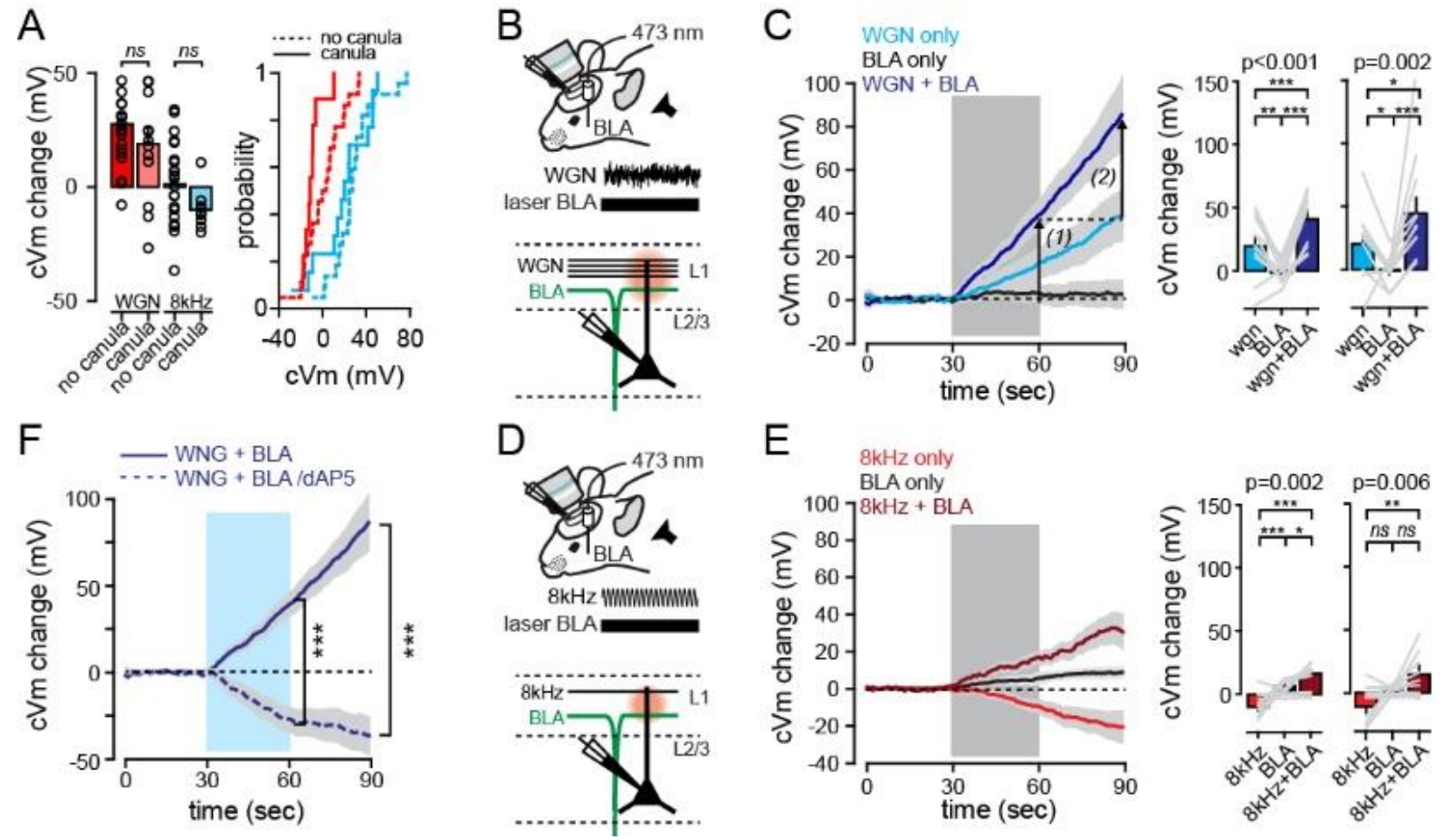

Fig. S9. The activation of BLA-to-FrA axons supports the non-linear integration of auditory tones

1055 A) Optical fibers implantation (canula) do not affect auditory-induced cVm changes as compared to non-implanted mice (no canula).

B) Co-activation protocol. ChR2-expressing BLA neurons were photo-stimulated during $W G N$ auditory stimulation.

C) Averaged cVm changes ( \pm sem) in response to BLA photo-stimulation alone (black), $W G N$ presentation alone (light blue) and WGN+BLA (dark blue). Arrows, analysis time points 1 (end of stimulation) and 2 (30 sec later). ${ }^{*}, \mathrm{p}<0.05 ;{ }^{* *}, \mathrm{p}<0.01 ;{ }^{* *}, \mathrm{p}<0.001 ;$ HolmSidak multiple comparisons after one-way anova $(\mathrm{p}<0.001$ for time point $1 ; \mathrm{p}=0.002$ for time point 2).

D, E) Same presentation as in (B, C) but for $8 \mathrm{kHz}$.

1065 F) Averaged cVm change ( \pm sem) during $W G N+B L A$ co-stimulation in controls (solid line) and after dAP5 application (dashed line). ${ }^{* * *}, \mathrm{p}<0.001 ; t$-test. 
A
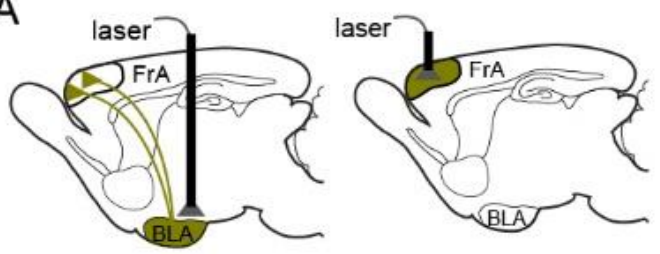

B
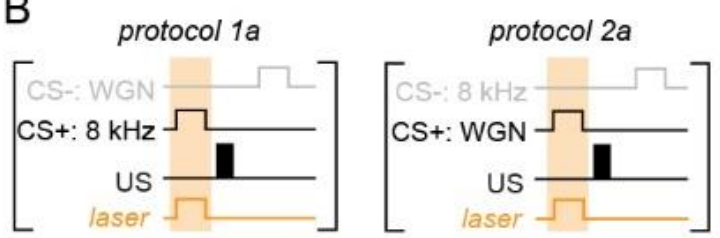

protocol $1 b$
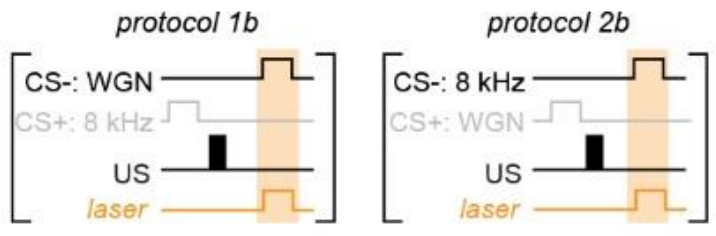

C

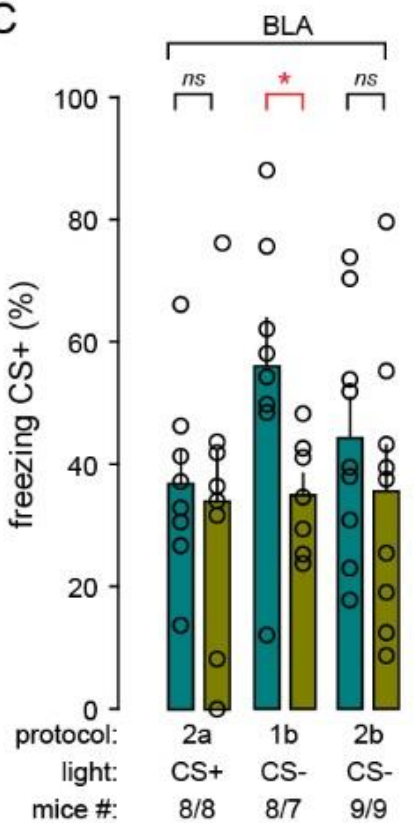

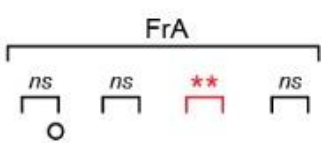

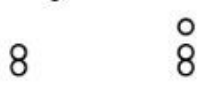

$\circ 8$

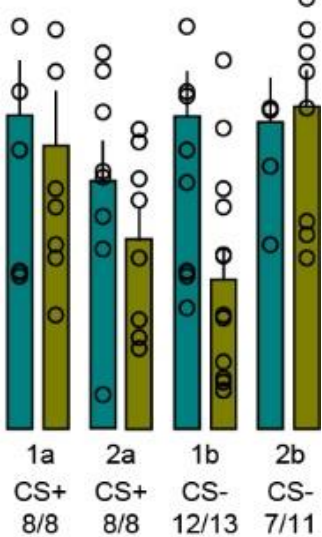

D FrA

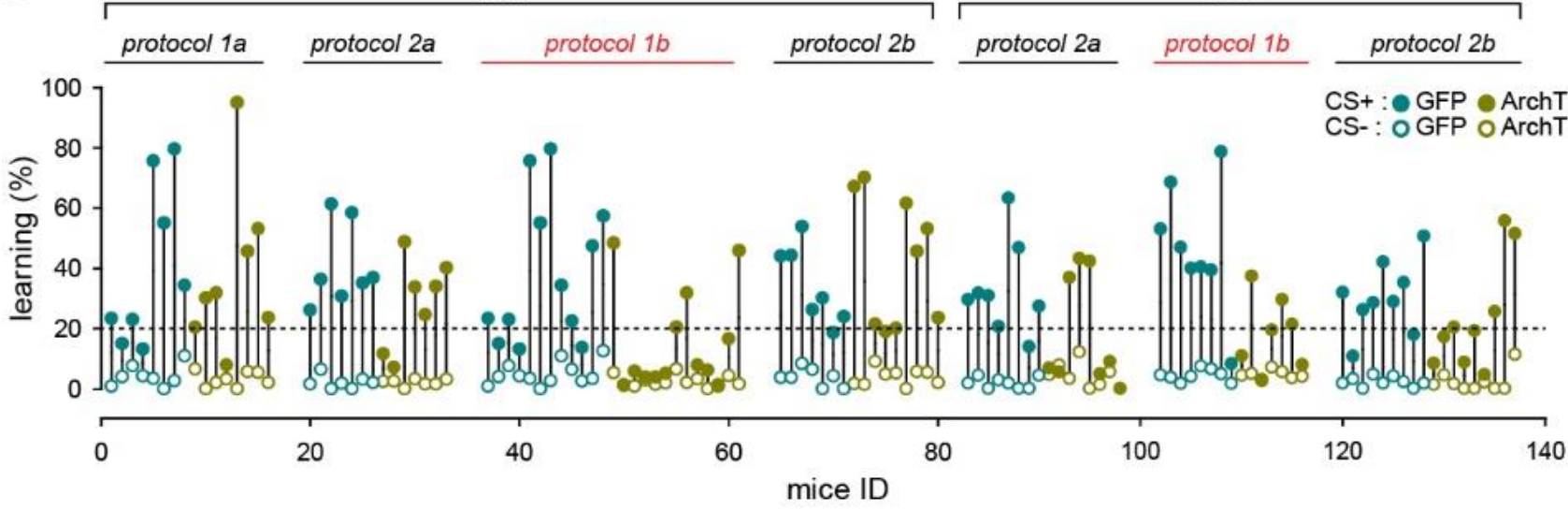

Fig. S10. Statistical comparisons between all behavioral optogenetic experiments

A) Experimental strategies. Left, mice were infected with AAV9.CBA.Flex.ArchT ( $\mathrm{n}=24)$ or AAV9.CAG.flex.eGFP (for controls, $n=25$ ) in BLA and CAV2.CMV.Cre in FrA, and chronically implanted with optical fibers bilaterally in BLA. Right, mice were infected with AAV9CAG-ArchT $(n=40)$ or AAV9-CaMKII-eGFP for controls $(n=35)$ in FrA, and chronically implanted with optical fibers bilaterally in FrA.

1075 B) Fear conditioning protocols. FrA neurons expressing ArchT (or GFP) were photostimulated during the presentation of $\mathrm{CS}+(8 \mathrm{kHz}$ for protocol 1a or $W G N$ for protocol 2a) or CS- (WGN for protocol $1 \mathrm{~b}$ or $8 \mathrm{kHz}$ for protocol 2b). US, footshock. Photo-simulations (orange box) were given during CS+ (protocols $1 \mathrm{a}$ and $2 \mathrm{a}$ ) or CS- (protocols $1 \mathrm{~b}$ and $2 \mathrm{~b}$ ). C) Freezing responses during recall upon CS+ for all behavioral conditions.

1080 C) Learning index during recall upon CS+ (filled circles) and CS- (open circles) in GFP (turquoise) and ArchT (khaki) expressing mice. Significant differences between GFP and ArchT-mice were seen only for protocol $1 \mathrm{~b}$ (red). 


\begin{tabular}{|c|c|c|c|c|c|c|c|c|c|c|}
\hline fig. & variable /units & group & $\mathbf{N}$ & mean & Std dev & median & $25 \%$ & $75 \%$ & test & p-value \\
\hline \multirow{6}{*}{ 1D } & WGN cVm change & control & 22 & 27.62 & 19.741 & & & & One way anova & $\mathrm{p}<0.001$ \\
\hline & (time point1) & dAP5 & 14 & -13.4 & 17.589 & & & & with multiple comparisons & $p<0.001$ (control vs dAP5) \\
\hline & & iMK801 & 5 & -2.24 & 5.368 & & & & (Holm-Sidak method) & $p=0.002($ control vs iMK801) \\
\hline & $\begin{array}{l}\text { WGN cVm change } \\
(\mathrm{mV})\end{array}$ & control & 22 & & & 25.653 & 14.3 & 41.2 & $\begin{array}{l}\text { Kruskal-Wallis one way } \\
\text { anova }\end{array}$ & $\mathrm{p}<0.001$ \\
\hline & (time point 2) & dAP5 & 14 & & & -13.74 & -19 & 0.93 & with multiple comparisons & $p<0.05$ (control vs dAP5) \\
\hline & & iMK801 & 5 & & & -0.263 & -16 & 4.81 & (Dunn's method) & $p<0.05$ (control vs iMK801) \\
\hline \multirow{4}{*}{$1 \mathrm{~F}$} & $\begin{array}{l}8 \mathrm{kHz} \text { cPSP change } \\
(\mathrm{mV})\end{array}$ & control & 22 & 1.16 & 18.145 & & & & One way anova & $\mathrm{p}=0.004$ \\
\hline & (end of stimulation) & dAP5 & 10 & -16.8 & 12.998 & & & & with multiple comparisons & $p=0.006$ (control vs dAP5) \\
\hline & (time point 2) & dAP5 & 10 & -15.4 & 15.447 & & & & with multiple comparisons & $p=0.012($ control vs dAP5) \\
\hline & & iMK801 & 5 & -22.7 & 12.508 & & & & (Holm-Sidak method) & $p=0.041($ control vs iMK801) \\
\hline \multirow{6}{*}{$2 \mathrm{D}$} & events per dendrites & baseline & 139 & 1.664 & 0.394 & & & & One way anova & $\mathrm{p}<0.001$ \\
\hline & during anesthesia & WGN & 139 & 3.282 & 0.864 & & & & repeated measures & $p<0.001$ (WGN vs baseline) \\
\hline & (number) & $8 \mathrm{kHz}$ & 139 & 1.19 & 0.813 & & & & with multiple comparisons & $p<0.001(W G N$ vs $8 k H z)$ \\
\hline & & $2 \mathrm{kHz}$ & 139 & 1.261 & 0.52 & & & & (Holm-Sidak method) & $p<0.001(W G N$ vs $2 \mathrm{kHz})$ \\
\hline & & & & & & & & & & $p=0.056(8 \mathrm{kHz}$ vs baseline $)$ \\
\hline & & & & & & & & & & $p=0.097(2 \mathrm{kHz}$ vs baseline $)$ \\
\hline \multirow{2}{*}{$2 \mathrm{~F}$} & & & & & & & & & & $p<0.001(2 \mathrm{kHz}$ vs baseline $)$ \\
\hline & & & & & & & & & & $p=0.128(8 \mathrm{kHz} v \mathrm{vs} 2 \mathrm{kHz})$ \\
\hline \multirow{4}{*}{ 2I } & events per dendrites & WGN+ & 139 & 3.282 & 0.864 & & & & Two way anova & $\mathrm{p}=0.026$ \\
\hline & $\begin{array}{l}\text { anesthesia(+) vs awake(- } \\
\text { ) }\end{array}$ & WGN- & 104 & 3.193 & 1.005 & & & & repeated measures & $p=0.844(W G N+v s W G N-)$ \\
\hline & (number) & pure+ & 139 & 1.226 & 0.659 & & & & with multiple comparisons & $p=0.037$ (pure + vs pure -$)$ \\
\hline & pure tones pooled & pure- & 104 & 2.241 & 0.5117 & & & & (Holm-Sidak method) & \\
\hline \multirow{4}{*}{$3 \mathrm{E}$} & Learning+ (total mice) & GFP+ & $6(8)$ & & & & & & pearson $\chi^{2}$ test $\left(\chi^{2}=0.4103\right)$ & $\mathrm{p}=0.522$ \\
\hline & protocol 1a & ArchT + & $7(8)$ & & & & & & & \\
\hline & Learning+ (total mice) & GFP+ & $7(8)$ & & & & & & pearson $\chi^{2}$ test $\left(\chi^{2}=1.3333\right)$ & $\mathrm{p}=0.248$ \\
\hline & protocol $2 \mathrm{a}$ & ArchT+ & $5(8)$ & & & & & & & \\
\hline \multirow{5}{*}{$3 \mathrm{D}$} & freezing $(\%)$ & CS- hab & 16 & 5.811 & 4.9544 & 5.236 & 1.58 & 8.58 & Two way anova & $\mathrm{p}<0.001$ (interaction) \\
\hline & GFP (all protocols) & CS- rec & 16 & 5.11 & 5.9977 & 2.833 & 1.04 & 7.83 & repeated measures & $p=0.8(C S+h a b$ vs CS $-h a b)$ \\
\hline & hab=habituation & $\mathrm{CS}+$ hab & 16 & 5.33 & 3.9664 & 5.015 & 1.91 & 7.34 & with multiple comparisons & $p<0.001(C S+$ rec vs $C S-r e c)$ \\
\hline & rec=recall & $\mathrm{CS}+\mathrm{rec}$ & 16 & 48.48 & 19.608 & 45.5 & 30.5 & 60.5 & (Holm-Sidak method) & $p=0.99(C S-$ hab vs CS-rec) \\
\hline & & & & & & & & & & $p<0.001(C S+h a b$ vs $C S+r e c)$ \\
\hline
\end{tabular}


bioRxiv preprint doi: https://doi.org/10.1101/569137; this version posted February 8, 2020. The copyright holder for this preprint (which was not certified by peer review) is the author/funder. All rights reserved. No reuse allowed without permission.

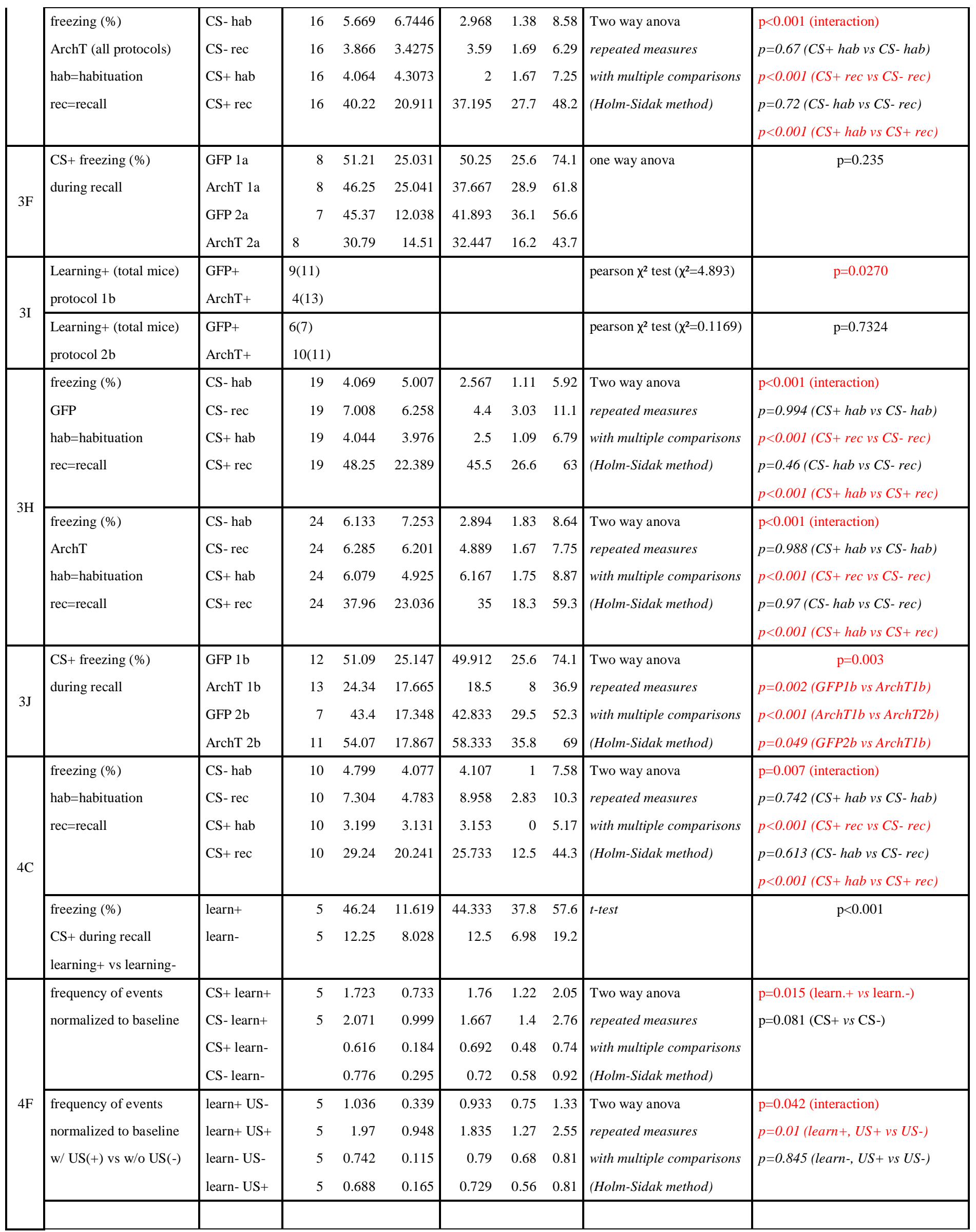


bioRxiv preprint doi: https://doi.org/10.1101/569137; this version posted February 8, 2020. The copyright holder for this preprint (which was not certified by peer review) is the author/funder. All rights reserved. No reuse allowed without permission.

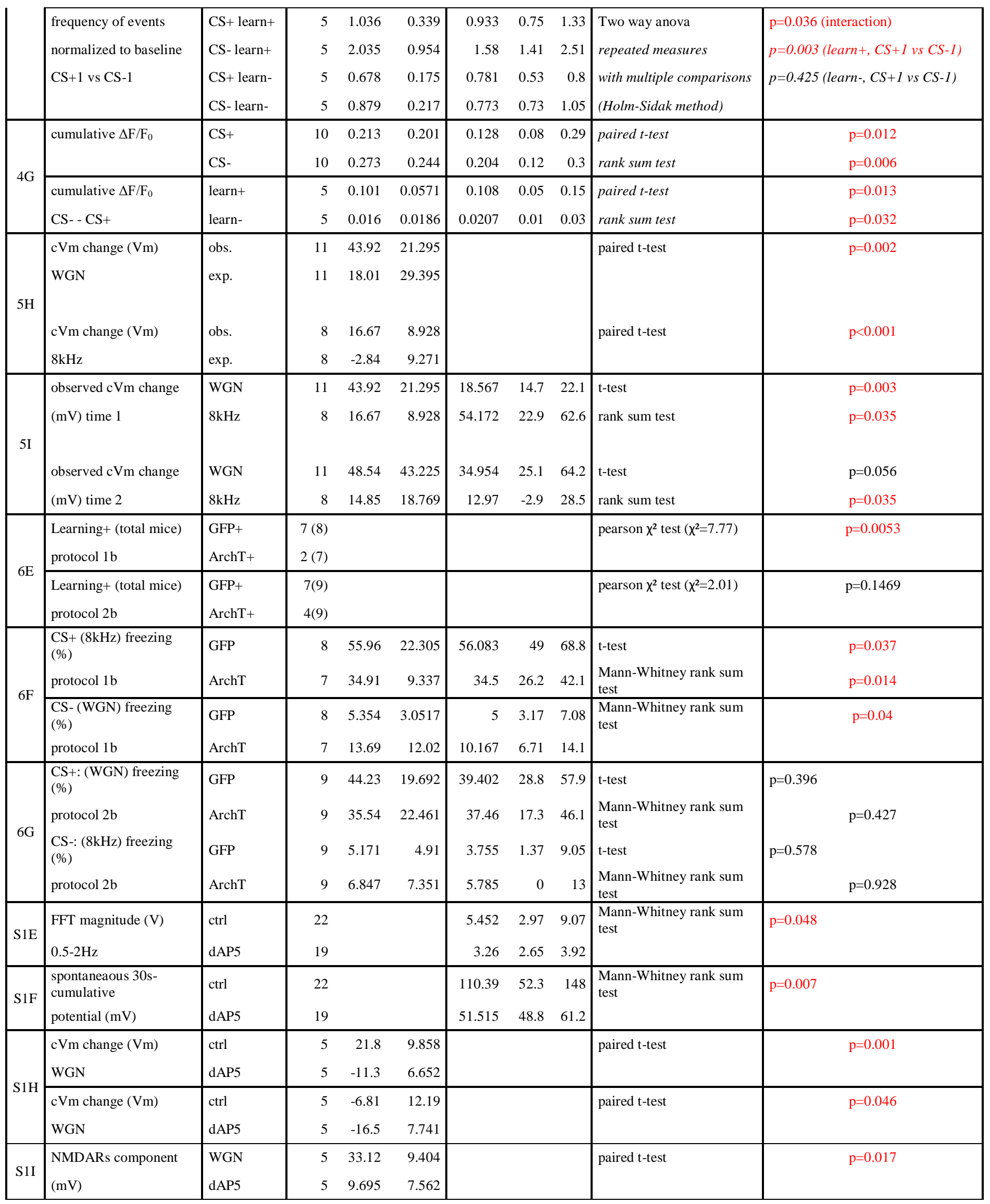


bioRxiv preprint doi: https://doi.org/10.1101/569137; this version posted February 8, 2020. The copyright holder for this preprint (which was not certified by peer review) is the author/funder. All rights reserved. No reuse allowed without permission.

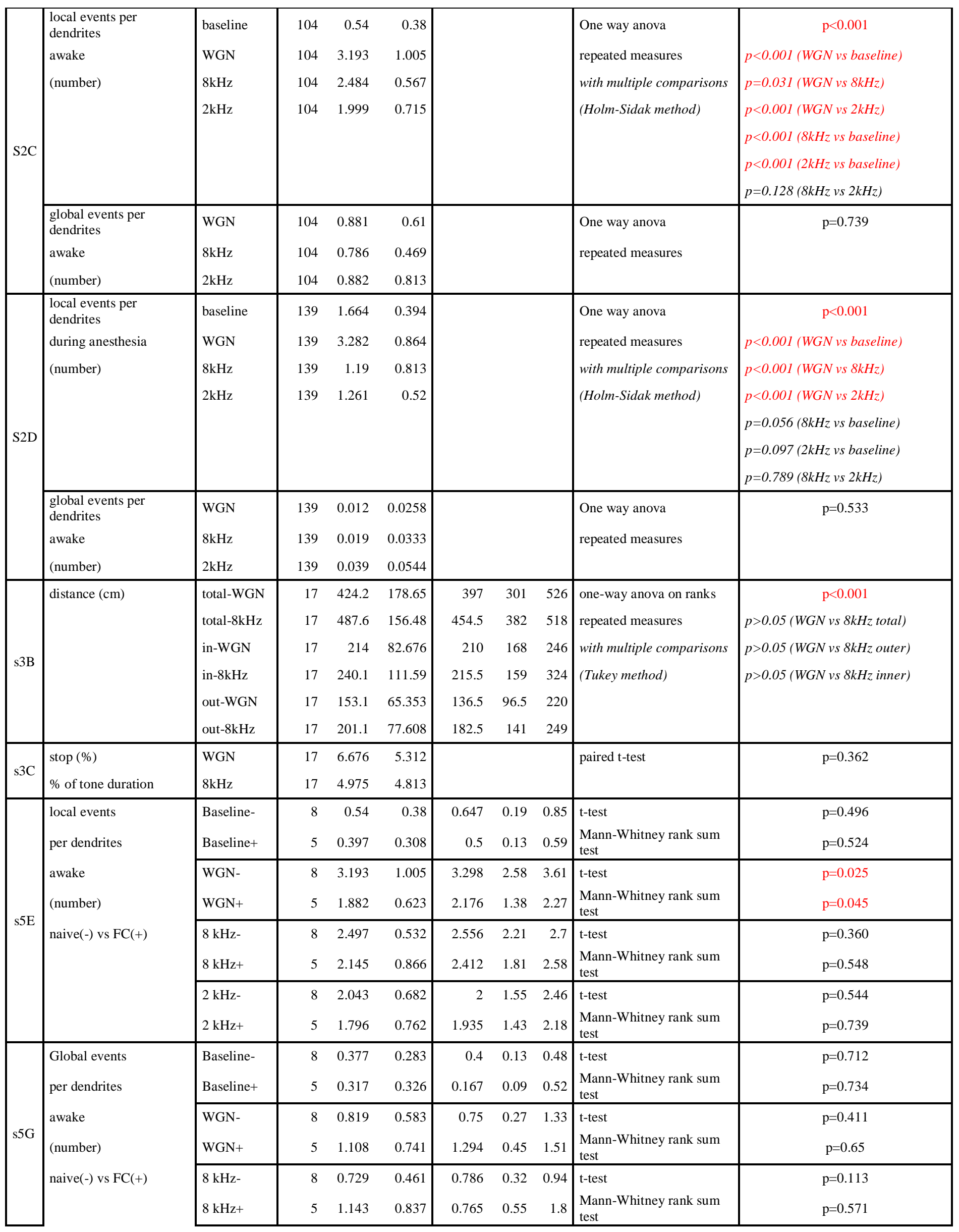




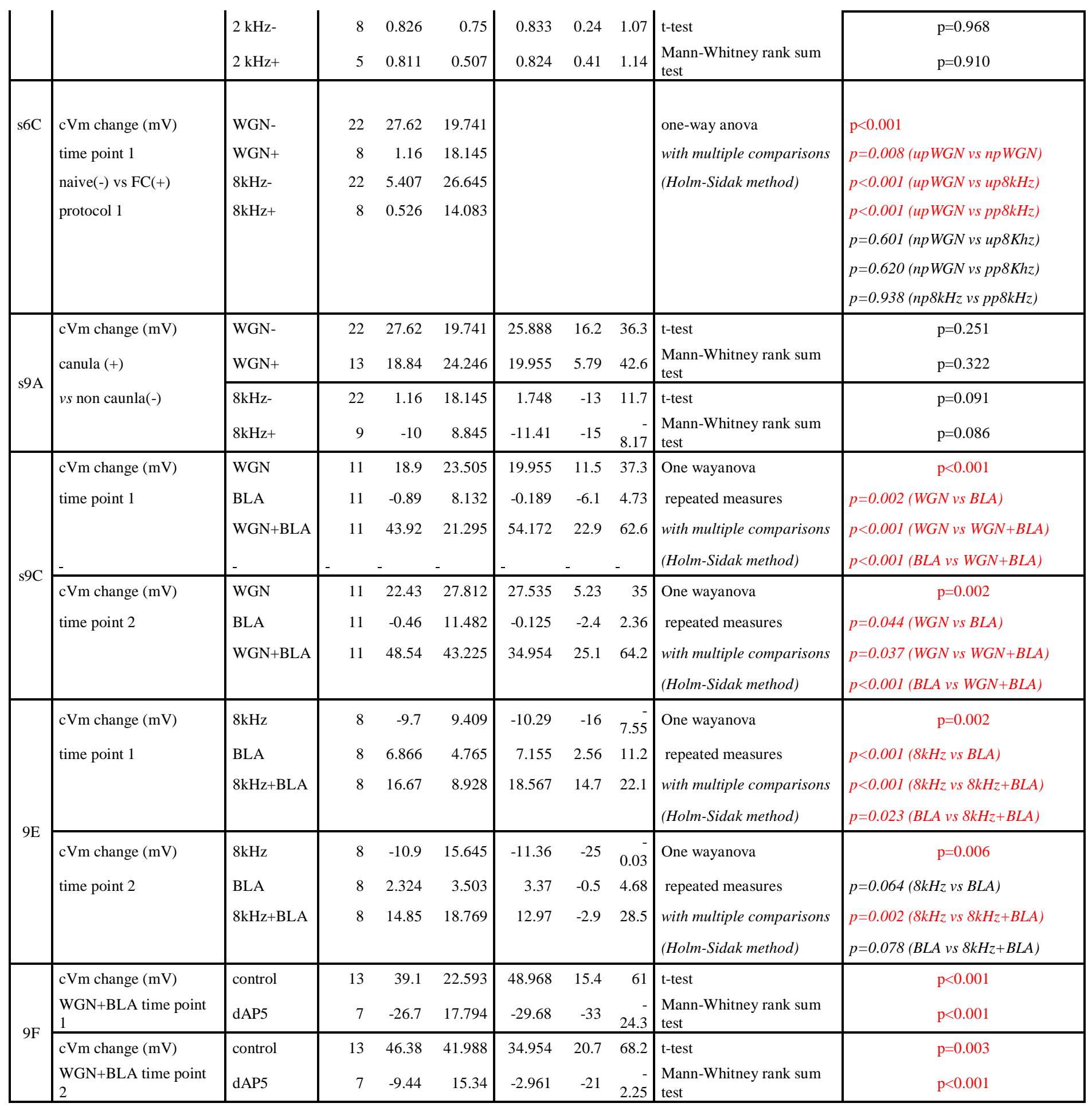

\section{Supplementary Table 1. Data and Statistic}

1085 All statistics were performed using Matlab (Mathworks) with an $\alpha$ significant level set at 0.05 (in red within the table). Normality of all value distributions and the equality of variance between different distributions were first assessed by the Shapiro-Wilk and Levene median tests, respectively. Standard parametric tests were used when data passed the normality and equal variance tests. Non-parametric tests were used otherwise. Only 
bioRxiv preprint doi: https://doi.org/10.1101/569137; this version posted February 8, 2020. The copyright holder for this preprint (which was not certified by peer review) is the author/funder. All rights reserved. No reuse allowed without permission.

1090 two-sided tests were used. When applicable, pair-wise multiple post-hoc comparisons were done by using the Holm-Sidak method. 


\section{METHODS}

All experiments were performed in accordance with the Guide for the Care and Use of Laboratory Animals (National Research Council Committee (2011): Guide for the Care and Use of Laboratory Animals, 8th ed. Washington, DC: The National Academic Press.) and the European Communities Council Directive of September 22th 2010 (2010/63/EU, 74). Experimental protocols were approved by the institutional ethical committee guidelines for animal research (N50DIR_15-A) and by the French Ministry of Research $\left(\mathrm{N}^{\circ} 02169.01\right)$. We used male C57Bl6/J 6-weeks old mice from Charles River that were housed with littermates (3-4 mice per cage) in a 12-h light-dark cycle. Cages were enriched and food and water were provided ad libitum.

\section{Surgery and virus injection}

Mice were anesthetized with an intraperitoneal (i.p.) injection of a mix containing medetomidine (sededorm, $0.27 \mathrm{mg} \mathrm{kg}^{-1}$ ), midazolam ( $\left.5 \mathrm{mg} \mathrm{kg}^{-1}\right)$ and fentanyl $(0.05 \mathrm{mg} \mathrm{kg}$ ${ }^{1}$ ) in sterile $\mathrm{NaCl}$ 0.9\% (MMF-mix). Analgesia was achieved by local application of $100 \mu \mathrm{l}$ of lidocaine (lurocaine, 1\%) and subcutaneous (s.c.) injection of buprenorphine (buprécare, $0.05 \mathrm{mg} \mathrm{kg}^{-1}$ ). $40 \mu \mathrm{l}$ of dexamethasone (dexadreson, $0.1 \mathrm{mg} \mathrm{ml}^{-1}$ ) was administrated intramuscularly (i.m.) in the quadriceps to prevent inflammation potentially caused by the friction of the drilling. A heating-pad was positioned underneath the animal to keep the body temperature at $37^{\circ} \mathrm{C}$. Eye dehydration was prevented by topical application of ophthalmic gel. The skin above the skull was disinfected with modified ethanol $70 \%$ and betadine before an incision was made. Stereotaxic injections were done as previously described (Gambino et al., 2014). Briefly, the bregma and lambda were aligned ( $\mathrm{x}$ and $\mathrm{z}$ ) and a hole for injection was made using a pneumatic dental drill (BienAir Medical Technologies, AP-S001). The injections were targeted either to the layer 2/3 of the FrA (from bregma: AP, $+2.8 \mathrm{~mm}$; DV, $-0.2-0.3 \mathrm{~mm}$; ML $\pm 1.0 \mathrm{~mm}$ ) or to the BLA (from bregma: AP, $-1.3 \mathrm{~mm}$; DV, -4.5 to $4.8 \mathrm{~mm}$; ML, $\pm 2.9 \mathrm{~mm}$ ), or to both at the same time. $200 \mathrm{nl}$ of virus were injected at a maximum rate of $60 \mathrm{nl} / \mathrm{min}$, using a glass pipette (Wiretrol, Drummond) attached to an oil hydraulic manipulator (MO-10, Narishige).

The following viruses were used depending on the experiments. AAV-ChR2 (AAV9.CamKIIa.hChR2(H134R).eYFP.WPRW.SV40, Penn Vector Core) was unilaterally injected in the right BLA, whereas AAV-ArchT-Flex (AAV9.CBA.flex.Arch-GFP.WPRE.SV40, 
Penn Vector Core) and CAV2-Cre (Cav2.CMV.Cre, IGMM BioCampus Montpellier) were bilaterally injected into the BLA and FrA, respectively. Control experiments were performed using an AAV containing the DNA construct for GFP (AAV9.CAG.flex.eGFP.WPRE.bGH). For axonal calcium imaging, AAV-GCaMP6f (AAV1.Syn.GCaMP6f.WPRE.SV40, Penn Vector Core) was injected to the right BLA. For dendritic calcium imaging, AAV-GCaMP6s (AAV9.Syn.Flex.GCaMP6s.WPRE.SV40, Penn Vector Core) and a 1:10000 dilution of AAV-Cre (AAV1.hSyn.Cre.WPRE.hGH, Penn Vector to diffuse for at least $10 \mathrm{~min}$ before the pipette was withdrawn. Mice were then either prepared for cranial window implantation or waked-up by a sub-cutaneous injection of a mixture containing atipamezole (revertor, $\left.2.5 \mathrm{mg} \mathrm{kg}^{-1}\right)$, flumazenil $\left(0.5 \mathrm{mg} \mathrm{kg}^{-1}\right)$, and buprenorphine (buprécare, $0.1 \mathrm{mg}$ kg-1) in sterile $\mathrm{NaCl}$ 0.9\% (AFB-mix).

The cranial windows were made as previously described (Gambino et al., 2014). Briefly, after skull's exposure a $\sim 5 \mathrm{~mm}$ plastic chamber was attached on the area of interest and a $3 \mathrm{~mm}$ craniotomy was made on the right hemisphere above FrA and M2, with a pneumatic dental drill, leaving the dura intact. The craniotomy was covered with sterile saline $(0.9 \% \mathrm{NaCl})$ and sealed with a $3 \mathrm{~mm}$ glass cover slip after viral injection (for imaging experiments). The chamber, the cover slip and a custom-made stainless steel head stage were well attached to the skull using dental acrylic and dental cement (Jet Repair Acrylic, Lang Dental Manufacturing).

To evaluate the viral expression profiles in BLA and FrA, fixed brain slices were imaged post-hoc using a wide-field epifluorescence microscope (Nikon, Eclipse N-iU). Illumination was set such that the full dynamic range of the 16-bit images was utilized. A two-dimensional graph of the intensities of pixel was plot using Fiji Software. 16-bit images' brightness was processed and masks were registered to the corresponding coronal plates (ranging from -1.94 to $-2.70 \mathrm{~mm}$ ) of the mouse brain atlas using Illustrator (Adobe), at various distances anterior (FrA) or posterior (BLA) to the bregma.

\section{0 \\ Fear conditioning and quantification of learning}

At least 5 days before starting behavioral experiments, mice went through handling with the same experimenter that performed the experiments in order to decrease stress. For consistency across experiments, mice were then habituated to 
auditory tones during 3 successive days. During habituation, mice were placed on the conditioning compartment (context A, consisting of a squared box with a grid floor that allows the delivery of a foot shock and with home cage litter under; cleaned between individuals with 70\% ethanol). Two conditional auditory stimuli (CS) (8 kHz pure tone; and white Gaussian noise (WGN); each composed of 27 pips, $50 \mathrm{~ms}$ in duration, $0.9 \mathrm{~Hz}$ for 30 s) were presented 4 times with a $80 \mathrm{~dB}$ sound pressure level and variable inter stimulus interval (ISI). The freezing time during each CS presentation was measured and the mice returned to their home cage. Mice were fear conditioned 24 hours after the last habituation phase by using a classical differential protocol. Briefly, mice were exposed to context $A$ and 5 auditory tones (CS+) were paired with the unconditional stimulus (US, $1 \mathrm{~s}$ foot-shock, $0.6 \mathrm{~mA}$ ). The onset of US coincided with the CS+ offset. 5 CS- presentations were intermingled with CS+ presentations with a variable (10-60 s) ISI. CS were counterbalanced with WGN and $8 \mathrm{kHz}$ pure tones being used as CS+ and CS-, respectively. Recall tests were carried out 24, 48 and 72 hours after the conditioning phase by measuring the freezing time during the presentation of $2 \mathrm{CS}+$ and $2 \mathrm{CS}$ - in a new context (context B, consisting of a cylindrical white compartment with home cage litter on the floor; cleaned between individuals with septanios MD 2\%).

For optogenetic experiments using archeorhodopsin (ArchT) or GFP controls, mice were subjected to the same behavioral protocol described above. Optogenetic inhibition of FrA neurons or BLA-to-FrA projections upon CS presentation was achieved during the conditioning phase by synchronizing each pip (50 ms) composing the CS+ or the CS- with a $50 \mathrm{~ms}$-laser pulse. For the experiments in which the conditioning phase was taken place under the 2 photon microscope, the context consisted of the microscope shading box in which the mice were head-restrained in a custom tube containing a shocking grid at the bottom. CS and US presentations were triggered by a MATLAB routine, associated to a pulse-stimulator (Master-8, A.M.P.I) capable of triggering the foot shock. For somatic and dendritic calcium imaging experiments, behavior was assessed at least 6 hours after imaging sessions. For whole-cell recordings experiments, mice were anesthetized and prepare for patch recordings immediately after behavior.

For each behavioral session, the total time duration ( $\mathrm{sec}$ ) of freezing episodes upon CS+ and CS- presentation was quantified automatically using a fire-wire CCD-camera connected to an automated freezing detection software (AnyMaze, Ugo Basile, Italy), and 
expressed as \% of freezing. Learning index was further quantified for each CS by multiplying the \% of freezing in each condition by the corresponding index of discrimination by using the following equation:

$$
\text { learning index }(\%)=\text { freezing }(\%) \times \frac{\text { freezing } C S^{+}(\%)-\text { freezing } C S^{-}(\%)}{\text { freezing } C S^{+}(\%)+\text { freezing } C S^{-}(\%)}
$$

Learning index $<20 \%$ during recall was considered as a failure of conditioning.

\section{In vivo whole cell recordings}

Isoflurane ( $4 \%$ with $\sim 0.5 \mathrm{I} \mathrm{min}^{-1} \mathrm{O}_{2}$ ) combined with an i.p. injection of urethane (1.5 $\mathrm{g} \mathrm{kg}^{-1}$, in lactated ringer solution containing in [mM] $102 \mathrm{NaCl}, 28 \mathrm{Na}$ L Lactate, $4 \mathrm{KCl}$, $\left.1.5 \mathrm{CaCl}_{2}\right)$ was used to induce anesthesia and prolonged by supplementary urethane 0.15 $\mathrm{g} \mathrm{kg}^{-1}$ ) if necessary. To prevent risks of inflammation, brain swelling and salivary excretions, $40 \mu \mathrm{l}$ of dexamethasone (dexadreson, $0.1 \mathrm{mg} \mathrm{ml}^{-1}$, i.m.) and glycopyrrolate (Robinul-V, $0.01 \mathrm{mg} \mathrm{kg}^{-1}$, s.c.) were injected before the surgery. Adequate anesthesia (absence of toe pinch and corneal reflexes, and vibrissae movements) was constantly checked and body temperature was maintained at $37^{\circ} \mathrm{C}$ using a heating-pad positioned underneath the animal. Ophthalmic gel was applied to prevent eye dehydration. Analgesia was provided as described for viral injection (with lidocaine and buprenorphine). After disinfection of the skin (with modified ethanol 70\% and betadine), the skull was exposed and a $\sim 3 \mathrm{~mm}$ plastic chamber was attached to it above the prefrontal cortex using a combination of super glue (Loctite) and dental acrylic and dental cement (Jet Repair Acrylic, Lang Dental Manufacturing). A small $\sim 1 \mathrm{x} 1 \mathrm{~mm}$ craniotomy centered above the FrA ( $+2.8 \mathrm{~mm}$ from bregma, $\pm 1.0 \mathrm{~mm}$ midline) was made using a pneumatic dental drill, leaving the dura intact.

Whole-cell patch-clamp recordings of L2/3 pyramidal neurons were obtained as previously described (Gambino et al., 2014) Briefly, high-positive pressure (200-300 mbar) was applied to the pipette (5-8 $\mathrm{M} \Omega$ ) to prevent tip occlusion, when passing the pia. Immediately after, the positive pressure was reduced to prevent cortical damage. The 
pipette resistance was monitored in the conventional voltage clamp configuration during the descendent pathway through the cortex (until $-200 \mu \mathrm{m}$ from the surface) of $1 \mu \mathrm{m}$ steps. When the pipette resistance abruptly increased, the 3-5 G $\Omega$ seal was obtained by decreasing the positive pressure. After break-in, Vm was measured, and dialysis was allowed to occur for at least $5 \mathrm{~min}$ before launching the recording protocols. Currentclamp recordings were made using a potassium-based internal solution (in mM: 135 potassium gluconate, $4 \mathrm{KCl}, 10 \mathrm{HEPES}, 10 \mathrm{Na}$-phosphocreatine, 4 Mg-ATP, 0.3 Na-GTP, and $25 \mu \mathrm{M}, \mathrm{pH}$ adjusted to 7.25 with $\mathrm{KOH}, 285 \mathrm{mOsM}$ ), and acquired using a Multiclamp 700B Amplifier (Molecular Devices). Spontaneous activity was recorded prior, during and after the presentation of auditory stimulation. Spiking pattern of patched cells was analyzed to identify pyramidal neurons. dAP5 (1 mM, Tocris) was topically applied to the dura mater, before whole cell recordings. Offline analysis was performed using custom routines written in Sigmaplot (Systat), IGOR Pro (WaveMetrics) and Matlab (Mathworks).

\section{In vivo optogenetics}

After virus injection for ChR2 or ArchT expression, mice were subsequently implanted with fiber optic cannula for optogenetics (CFML22U, Thorlabs) in the BLA. The optic fibers were previously cleaved with a fiber optic scribe (S90R, Thorlabs) at $4.5 \mathrm{~mm}$ for BLA. The cannula were guided and stereotaxically inserted inside the brain with the help of a cannula holder (XCL, Thorlabs) through the same burr hole used for the viral injections (BLA coordinates from bregma: AP, $-1.3 \mathrm{~mm}$; DV, $-4.5 \mathrm{~mm}$; ML, $\pm 2.9 \mathrm{~mm}$ ) and secured in place with a mix of super glue (Loctite) and dental acrylic and dental cement (Jet Repair Acrylic, Lang Dental Manufacturing). Anesthesia was reversed using AFB-mix for mice assigned to behavioral experiments. For in vivo photostimulation of ChR2expressing BLA neurons, the fiber optic cannula and the optogenetic patch cable (M83L01, Thorlabs) were connected through a ceramic split mating sleeve (ADAL1, Thorlabs). The patch cable was then coupled to a blue DPSS laser (SDL-473-050MFL, Shanghai Dream Lasers Technology) which was triggered by a pulse-stimulator (Master9, A.M.P.I), able to synchronize $50 \mathrm{~ms}$ laser pulses with $50 \mathrm{~ms}$ sound pips composing the CS. For inhibition of BLA-to-FrA projections during learning, in vivo bilateral optic stimulation of ArchT-expressing neurons was achieved by coupling the optic fibers implanted in BLA to a multimode fiber optic coupler (FCMH2-FCL, Thorlabs), with a 
ceramic split mating sleeve, and subsequently connected to a yellow DPSS laser (SDL-LH1500, Shanghai Dream Lasers Technology).

\section{In vitro whole-cell recordings}

Mice were anesthetized with a mixture of ketamine/xylazine $(100 \mathrm{mg} / \mathrm{kg}$ and $10 \mathrm{mg} / \mathrm{kg}$ respectively) and cardiac-perfused with ice-cold, oxygenated (95\% 02, 5\% C02) cutting solution (NMDG) containing (in mM): 93 NMDG, $93 \mathrm{HCl}, 2.5 \mathrm{KCl}, 1.2$ NaH2P04, 30 NaHCO3, 25 Glucose, 10 MgSO4, 0.5 CaCl2, 5 Sodium Ascorbate, 3 Sodium Pyruvate, 2 Thiourea and 12mM N-Acetyl-L-cysteine (pH 7.3-7.4, with osmolarity of 300310 mOsm). Brains were rapidly removed and placed in ice-cold and oxygenated NMDG cutting solution (described above). Coronal slices $(300 \mu \mathrm{m})$ were prepared using a Vibratome (VT1200S, Leica Microsystems, USA) and transferred to an incubation chamber held at $32^{\circ} \mathrm{C}$ and containing the same NMDG cutting solution. After this incubation (9-11 $\mathrm{min})$, the slices were maintained at room temperature in oxygenated modified ACSF containing (mM): $92 \mathrm{NaCl}, 2.5 \mathrm{KCl}, 1.2 \mathrm{NaH} 2 \mathrm{PO} 4,30 \mathrm{NaHCO}, 20$ HEPES, 25 Glucose, 2 MgSO4, 2 CaCl2, 5 Sodium Ascorbate, 3 Sodium Pyruvate, 2 Thiourea and $12 \mathrm{mM}$ N-Acetyl-L-cysteine (pH 7.3-7.4, with osmolarity of 300-310 mOsm) until recording.

Whole-cell recordings of layer 2/3 FrA principal neurons were performed on coronal slices (from bregma: $+2.58 \mathrm{~mm}$ to $+3.08 \mathrm{~mm}$ ) at $30-32^{\circ} \mathrm{C}$ in a superfusing chamber. Patch electrodes (3-5 M $\Omega$ ) were pulled from borosilicate glass tubing and filled with a K-gluconate-based intracellular solution (in mM: $140 \mathrm{~K}$-gluconate, 5 QX314-Cl, 10 HEPES, 10 phosphocreatine, 4 Mg-ATP and 0.3 Na-GTP (pH adjusted to 7.25 with $\mathrm{KOH}$, 295 mOsm). BLA-to-FrA monosynaptic EPSCs were elicited by 1-50 ms light stimulations delivered by an ultrahigh power $460 \mathrm{~nm}$ LED (Prizmatix Ltd, Israel). Data were recorded with a Multiclamp700B (Molecular Devices, USA), filtered at $2 \mathrm{kHz}$ and digitized at $10 \mathrm{kHz}$. Data were acquired and analysed with pClamp10.2 (Molecular Devices).

\section{2-photon laser-scanning microscope (2PSLM)-based calcium imaging}

Head-fixed awake mice were placed and trained under the microscope every day for at least 7 days prior to the experiment, and then imaged 21 to 35 days after virus 
injection using an in vivo non-descanned FemtoSmart 2PLSM (Femtonics, Budapest, Hungary) equipped with a $\times 16$ objective (0.8 NA, Nikon). The MES Software (MES v.4.6; Femtonics, Budapest, Hungary) was used to control the microscope, the acquisition parameters, and the TTL-driven synchronization between the acquisition and auditory/footshock stimuli. The GCaMPs were excited using a Ti:sapphire laser operating at $\lambda=910 \mathrm{~nm}$ (Mai Tai DeepSee, Spectra-Physics) with an average excitation power at the focal point lower than $50 \mathrm{~mW}$. Time-series images were acquired within a field-of-view of $300 \times 300 \mu \mathrm{m}$ (256 lines, 1ms/line) for axons; for dendrite: $200 \times 60 \mu \mathrm{m}$ (64 lines, $0.5 \mathrm{~ms} /$ line). Each imaging session consisted of $30 \mathrm{~s}$ of baseline recording followed by 8 gaussian and 8 pure (8kHz)-tone auditory stimuli delivered with pseudo-random delays. We imaged on average 3500 frames ( 900 s) per session, and no visible photo-bleaching was observed. Images were then analyzed as previously described (Gambino et al., 2014) using custom routines written in Fiji and Matlab (Mathworks). We registered images over time and corrected XY motion artifacts within a single imaging session by using crosscorrelation based on rigid body translation (Stack aligner, Image J, NIH, USA). Motion corrections were then assessed by computing pair-wise 2D correlation coefficient (Image correlation, Image J, NIH, USA), and frames were discarded from the analysis if lower than 0.7. Regions of interest (ROIs) for pyramidal neurons and putative axonal boutons were selected and drawn manually. All pixels within each ROI were first averaged providing a single time-series of raw fluorescence. To limit the effect of fluorescence drift over time, the baseline fluorescence $\left(F_{0}\right)$ was calculated as the mean of the lower $50 \%$ of previous 3 s fluorescence values. Change in fluorescence $\left(\Delta F_{t} / F_{0}\right)$ was defined as $\left(F_{t}-F_{0}\right) / F_{0}$, were $F_{t}$ is the fluorescence intensity at time $t$ (time of the first pixel in each frame). Calcium events were then detected using a template-based method with a custom library of calcium transients. Templates were created by extracting and averaging segments of data that were visually identified as corresponding to a transient. Calcium transients whose peak amplitude reached a $3 \mathrm{X}$ background standard deviation threshold were further considered for analysis. Each detected event was inspected visually and analysis was restricted to detected events rather than on raw fluorescence. For extracting spatial profiles of dendritic calcium events, small ROIs of 2 X 2 pixels are generated along the dendrite by using custom routine in Fiji. The spread of $\mathrm{Ca}^{2+}$ events was then quantified by calculating the full-width at half-max (fwhm, expressed as \% of total dendritic length) of the normalized Gaussian fit at the time when the averaged $\Delta \mathrm{F} / \mathrm{F}_{0}$ was maximal. 


\section{Data availability and Statistics}

All data generated or analyzed during this study are included in the manuscript, 1310 and provided in the supplementary statistical table. Experiments and analysis were conducted blind to the operator. Data are presented as the median \pm interquartile range or mean \pm sem (except where stated differently). All statistics were performed using Matlab (Mathworks) and Sigmaplot (Systat) with an $\alpha$ significant level set at 0.05 . Normality of all value distributions and the equality of variance between different distributions were first assessed by the Shapiro-Wilk and Levene median tests, respectively. Standard parametric tests were only used when data passed the normality and equal variance tests. Non-parametric tests were used otherwise. Only two-sided tests were used. When applicable, pair-wise multiple post-hoc comparisons were done by using the Holm-Sidak method. No statistical methods were used to estimate sample size, but $\beta$-power values were calculated for parametric tests. 